\title{
Measurement of $\mathrm{ZZ}$ production in the $\ell \ell \nu \nu$ final state with the ATLAS detector in pp collisions at $\sqrt{\mathrm{s}}=13 \mathrm{TeV}$
}

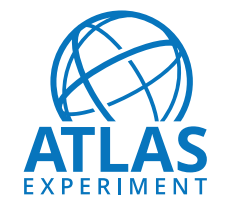

\section{The ATLAS collaboration}

\section{E-mail: atlas.publications@cern.ch}

ABSTRACT: This paper presents a measurement of $Z Z$ production with the ATLAS detector at the Large Hadron Collider. The measurement is carried out in the final state with two charged leptons and two neutrinos, using data collected during 2015 and 2016 in $p p$ collisions at $\sqrt{s}=13 \mathrm{TeV}$, corresponding to an integrated luminosity of $36.1 \mathrm{fb}^{-1}$. The integrated cross-sections in the total and fiducial phase spaces are measured with an uncertainty of $7 \%$ and compared with Standard Model predictions, and differential measurements in the fiducial phase space are reported. No significant deviations from the Standard Model predictions are observed, and stringent constraints are placed on anomalous couplings corresponding to neutral triple gauge-boson interactions.

KEYWORDS: Hadron-Hadron scattering (experiments)

ARXIV EPRINT: 1905.07163 


\section{Contents}

1 Introduction 1

2 ATLAS detector $\quad 4$

3 Data and simulation $\quad 5$

4 Selection of $\ell \ell \nu \nu$ events $\quad 7$

5 Total and fiducial phase spaces 10

6 Background estimation $\quad 12$

$\begin{array}{lll}7 & \text { Systematic uncertainties } & 15\end{array}$

8 Integrated cross-section results $\quad 16$

$9 \begin{array}{ll}\text { Differential cross-section results } & 19\end{array}$

$\begin{array}{ll}10 \text { Search for aTGCs } & 20\end{array}$

11 Conclusion $\quad 24$

$\begin{array}{ll}\text { The ATLAS collaboration } & 31\end{array}$

\section{Introduction}

In the Standard Model (SM), the production of gauge boson pairs has a profound connection with the non-Abelian nature of the electroweak (EW) theory and with the spontaneous breaking of the EW gauge symmetry. In addition, a broad range of new phenomena beyond the SM (BSM) are predicted to reveal themselves through diboson production. The study of diboson production probes a cornerstone of the EW theory and possible BSM physics scenarios, and it constitutes a salient component of the physics programme at the Large Hadron Collider (LHC). Among all the diboson processes, the production of two on-shell $Z$ bosons has the smallest cross-section, but is nevertheless quite competitive for measurements and searches, because of its generally good signal-to-background ratio for the fully leptonic decay channels. For instance, the $Z Z$ process is a leading channel to search for anomalous neutral triple-gauge-boson couplings (aTGCs) [1] and to study the off-shell production of the Higgs boson [2,3].

Figure 1 shows representative Feynman diagrams for $Z Z$ production at the LHC. The dominant process is $t$-channel production with a quark and anti-quark initial state, 


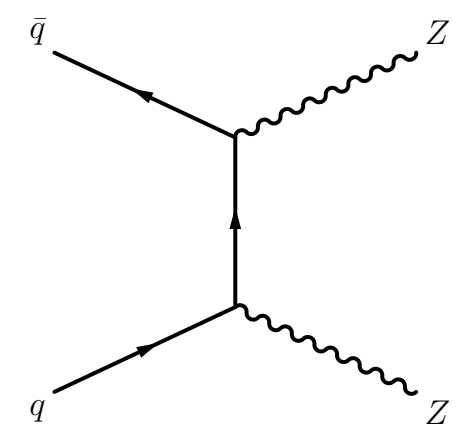

(a)

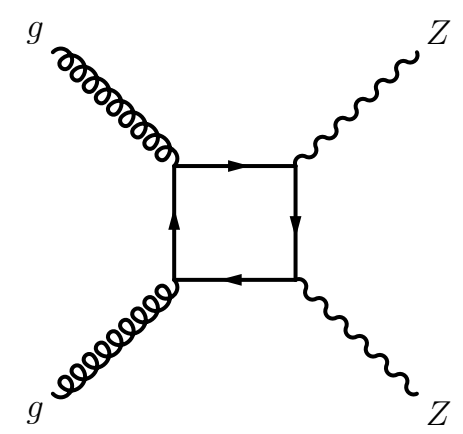

(d)

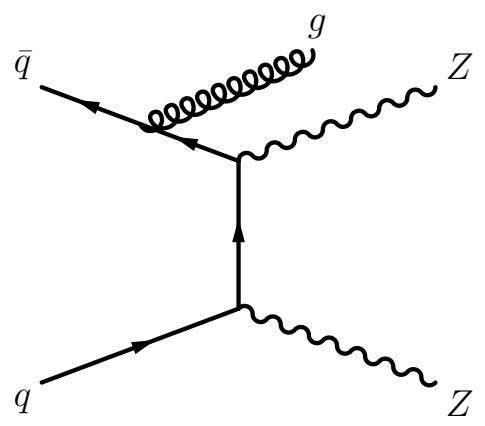

(b)

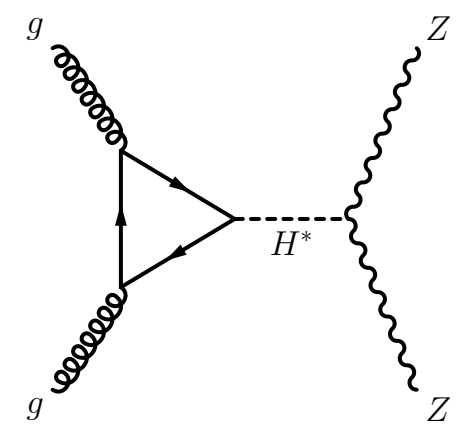

(e)

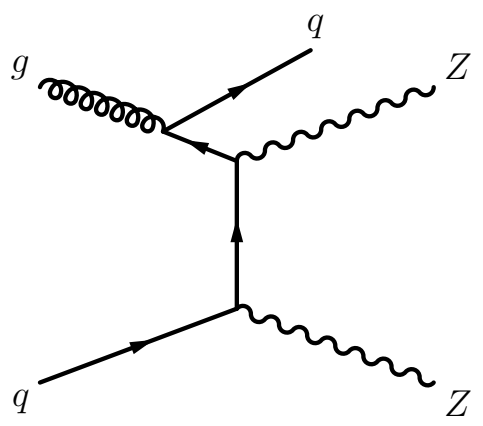

(c)

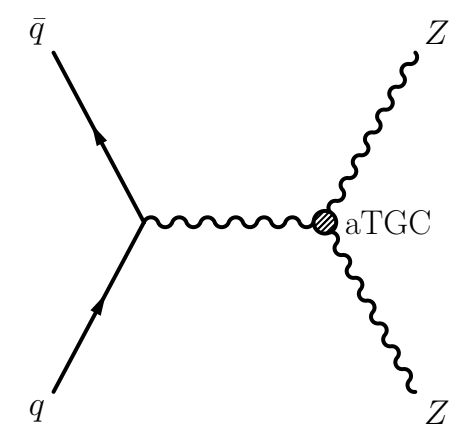

(f)

Figure 1. Representative Feynman diagrams for $Z Z$ production at the LHC: (a) lowest-order $t$-channel $q q Z Z$ production; (b) production of $Z Z$ plus one parton through the $q \bar{q}$ initial state; (c) production of $Z Z$ plus one parton through the $q g$ initial state; (d) $g g Z Z$ production with a fermion loop; (e) $g g Z Z$ production involving an exchange of a virtual Higgs boson; (f) $s$-channel production with aTGCs.

hereafter denoted by the $q q Z Z$ process. Higher-order QCD corrections to the $q q Z Z$ process are found to be sizeable [4], and two tree-level diagrams concerning production of two $Z$ bosons and one outgoing parton are shown. The gluon fusion process $(g g Z Z)$ includes two sub-processes, one with a fermion loop and the other involving a virtual Higgs boson. Although the $g g Z Z$ process only appears at $O\left(\alpha_{\mathrm{S}}^{2}\right)$, it nevertheless has a non-negligible contribution of $O(10 \%)$ to the total $Z Z$ production rate due to the large gluon flux at the LHC. The $s$-channel production is forbidden at the lowest order; however, the neutral TGCs can still acquire small values of $O\left(10^{-4}\right)$ in the SM, due to the correction with a fermion loop [5]. The observation of aTGCs with larger values would hint at the existence of new physics.

Measurements of $Z Z$ production at the LHC have been carried out in two decay final states, one with four charged leptons $(4 \ell)$ and the other with two charged leptons and two neutrinos $(\ell \ell \nu \nu)$. Using LHC Run-1 and Run-2 data, multiple results [6-12] have been reported by the ATLAS and CMS experiments. The most precise results to date have been obtained from the $4 \ell$ channel using $13 \mathrm{TeV}$ data $[8,12]$, where the integrated production cross-section has been measured to a precision of $5 \%$ and the upper bound on neutral aTGC parameters has been reduced to $10^{-3}$. The improved experimental precision 
has stimulated theoretical calculations with a greater accuracy, and the next-to-next-toleading-order (NNLO) QCD $[4,13,14,74,75]$ and next-to-leading-order (NLO) EW $[15,16]$ predictions have become available for the $q q Z Z$ process.

This paper presents a measurement of $Z Z$ production using $36.1 \mathrm{fb}^{-1}$ of data collected with the ATLAS detector in $p p$ collisions at $\sqrt{s}=13 \mathrm{TeV}$. This analysis is performed in the $\ell \ell \nu \nu(\ell=e$ or $\mu)$ final state, which has a larger branching fraction but suffers from higher background contamination in comparison with the $4 \ell$ channel. To ensure a good signal-tobackground ratio, the experimental selection requires one $Z$ boson boosted against the other in the transverse plane, which results in a pair of high- $p_{\mathrm{T}}$ isolated leptons and significant missing transverse momentum $\left(E_{\mathrm{T}}^{\mathrm{miss}}\right)$. The $\ell \ell \nu \nu$ channel thus offers higher data statistics than the $4 \ell$ channel for events with high- $p_{\mathrm{T}} Z$ bosons, and offers competitive precision for integrated and differential measurements, as well as good sensitivity to aTGCs.

The dominant background arises from $W Z$ production where the $Z$ boson decays into a pair of charged leptons. About $60 \%$ of the $W Z$ events which contribute to the $\ell \ell \nu \nu$ final state have the $W$ boson decaying leptonically $(W \rightarrow \ell \nu$ or $W \rightarrow \tau \nu \rightarrow \ell+3 \nu, \ell=e$ or $\mu$ ), where the final-state lepton escapes detection. The remaining $40 \% \mathrm{WZ}$ contribution is related to the $W \rightarrow \tau \nu$ decay with subsequent hadronic decays of the $\tau$-lepton. Another important background comes from the processes that genuinely produce the $\ell \ell \nu \nu$ final state but contain a lepton pair not originating directly from a $Z$-boson decay. This background, referred to as the non-resonant- $\ell \ell$ background, includes $W W$, top-quark ( $\overline{\mathrm{t}} \overline{\mathrm{t}}$ and $W t$ ), and $Z \rightarrow \tau \tau$ production. The production of a $Z$ boson in association with jet(s) $(Z+$ jets $)$ also constitutes a potentially large background source. The $Z+$ jets events with large "fake" $E_{\mathrm{T}}^{\mathrm{miss}}$ arise from heavy-flavour hadron decays in the accompanying jet(s), from jet mismeasurements in certain regions of the detector, and from the measurement resolution itself, owing to the additional $p p$ collisions in the same or neighbouring proton bunch crossings (pile-up). The $Z Z \rightarrow 4 \ell$ process yields a small contribution when one lepton pair misses detection, and it is considered as a background in this measurement. Finally, minor background contributions are expected from three-boson production ( $V V V$ with $V=W$ or $Z$ ) and production of $t \bar{t}$ accompanied by one or two vector bosons $(t \bar{t} V)$.

The integrated cross-section of $Z Z$ production is measured in a fiducial phase space and then extrapolated to a total phase space. The determination of the fiducial $\left(\sigma_{Z Z \rightarrow \ell \ell \nu \nu}^{\mathrm{fid}}\right)$ and total $\left(\sigma_{Z Z}^{\text {tot }}\right)$ cross-sections is obtained as shown in eq. (1.1):

$$
\sigma_{Z Z \rightarrow \ell \ell \nu \nu}^{\mathrm{fid}}=\frac{N_{Z Z}^{\mathrm{obs}}}{\mathcal{L} \times C_{Z Z}}, \quad \sigma_{Z Z}^{\mathrm{tot}}=\frac{N_{Z Z}^{\mathrm{obs}}}{\mathcal{L} \times C_{Z Z} \times A_{Z Z} \times B},
$$

where $C_{Z Z}$ stands for an overall efficiency correction factor, $A_{Z Z}$ is the fiducial acceptance, and $B$ is the branching fraction of the $Z Z \rightarrow \ell \ell \nu \nu(\ell=e, \mu)$ decay. The signal yield $N_{Z Z}^{\text {obs }}$ is determined through a fit to the observed $E_{\mathrm{T}}^{\text {miss }}$ spectrum, which leads to improved sensitivity compared with a simple event-counting method. The $A_{Z Z}\left(C_{Z Z}\right)$ factor is calculated as $N_{Z Z}^{\text {exp,fid }} / N_{Z Z}^{\text {exp,tot }}\left(N_{Z Z}^{\text {exp,det }} / N_{Z Z}^{\text {exp,fid }}\right)$, where $N_{Z Z}^{\text {exp,det }}, N_{Z Z}^{\text {exp,fid }}$, and $N_{Z Z}^{\text {exp,tot }}$ correspond to the expected signal yields for the $Z Z \rightarrow \ell \ell \nu \nu$ final state after the detectorlevel selection, in the fiducial region, and in the total phase space, respectively. The definitions of the total and fiducial phase spaces are elaborated in section 5 . The simulated 
events arising from the $Z Z \rightarrow \tau \tau \nu \nu$ decays with the subsequent $\tau \rightarrow \ell \nu \nu$ decays of both $\tau$-leptons are considered as signal events at detector level but excluded in the fiducial measurements. Throughout this paper, " $Z \rightarrow \ell \ell$ " denotes the decays of a $Z$ boson or a virtual photon into a charged-lepton pair.

Furthermore, differential cross-sections are reported in the fiducial region for eight kinematic variables, which are sensitive to effects from higher-order corrections and possible BSM physics. These variables include the transverse momentum of the leading lepton $\left(p_{\mathrm{T}}^{\ell 1}\right)$, the leading jet $\left(p_{\mathrm{T}}^{\mathrm{jet} 1}\right)$, the dilepton system $\left(p_{\mathrm{T}}^{\ell \ell}\right)$, and the $Z Z$ system $\left(p_{\mathrm{T}}^{Z Z}\right)$, the transverse mass of the $Z Z$ system $\left(m_{\mathrm{T}}^{Z Z}\right){ }^{1}$ the absolute rapidity of the dilepton system $\left(\left|y_{\ell \ell}\right|\right)$, the azimuthal angle difference between the two leptons $\left(\Delta \phi_{\ell \ell}\right)$, and the number of jets $\left(N_{\text {jets }}\right)$. Since no significant deviations from the SM are observed, upper limits are placed on the aTGC parameters [1], which typically manifest themselves as a signal excess growing rapidly as the partonic centre-of-mass energy $(\sqrt{\hat{s}})$ increases. In this analysis, aTGCs are searched for using the $p_{\mathrm{T}}^{\ell \ell}$ spectrum in the fiducial region, motivated by the fact that $p_{\mathrm{T}}^{\ell \ell}$ is correlated with $\sqrt{\hat{s}}$ and has a good experimental resolution.

\section{ATLAS detector}

The ATLAS detector [17-19] is a large multi-purpose detector with a forward-backward symmetric cylindrical geometry and nearly $4 \pi$ coverage in solid angle. ${ }^{2}$ It consists of an inner tracking detector surrounded by a thin superconducting solenoid, electromagnetic and hadronic calorimeters, and a muon spectrometer incorporating three large superconducting toroidal magnets each having eight coils assembled radially and symmetrically around the beam axis.

The inner-detector system (ID) is immersed in a $2 \mathrm{~T}$ axial magnetic field and provides charged-particle tracking in the range $|\eta|<2.5$. A high-granularity silicon pixel detector covers the vertex region and usually provides four measurements per track. The pixel detector is followed by a silicon microstrip tracker which usually provides four measurement points per track. These silicon detectors are complemented by a transition radiation tracker, which enables radially extended track reconstruction and improved momentum measurements up to $|\eta|=2.0$. The transition radiation tracker also provides electron identification information based on the fraction of hits (typically 30 hits in total) above a high-energy threshold designed for optimal electron-pion separation.

The calorimeter system covers the pseudorapidity range $|\eta|<4.9$. Within the region $|\eta|<3.2$, electromagnetic calorimetry is provided by barrel and endcap lead/liquid-argon (LAr) sampling calorimeters, with an additional thin LAr presampler covering $|\eta|<1.8$,

$$
{ }^{1} m_{\mathrm{T}}^{Z Z}=\sqrt{\left[\sqrt{m_{Z}^{2}+\left(p_{\mathrm{T}}^{\ell \ell}\right)^{2}}+\sqrt{m_{Z}^{2}+\left(E_{\mathrm{T}}^{\mathrm{miss}}\right)^{2}}\right]^{2}-\left|{\overrightarrow{p_{\mathrm{T}}}}^{\ell \ell}+\vec{E}_{\mathrm{T}}^{\mathrm{miss}}\right|^{2}} .
$$

${ }^{2}$ ATLAS uses a right-handed coordinate system with its origin at the nominal interaction point (IP) in the centre of the detector and the $z$-axis along the beam pipe. The $x$-axis points from the IP to the centre of the LHC ring, and the $y$-axis points upward. Cylindrical coordinates $(r, \phi)$ are used in the transverse plane, $\phi$ being the azimuthal angle around the $z$-axis. The pseudorapidity is defined in terms of the polar angle $\theta$ as $\eta=-\ln \tan (\theta / 2)$. 
to correct for energy loss in material upstream of the calorimeters. Hadronic calorimetry is provided by a steel/scintillating-tile calorimeter, segmented into three barrel structures within $|\eta|<1.7$, and two copper/LAr hadronic endcap calorimeters. The solid angle coverage is completed with forward copper/LAr and tungsten/LAr calorimeter modules optimised for electromagnetic and hadronic measurements, respectively.

The muon spectrometer (MS) comprises separate trigger and high-precision tracking chambers measuring the deflection of muons in a magnetic field generated by superconducting air-core toroids. The field integral of the toroids ranges between 2.0 and $6.0 \mathrm{~T} \cdot \mathrm{m}$ across most of the detector. A set of precision chambers covers the region $|\eta|<2.7$ with three layers of monitored drift tubes, complemented by cathode strip chambers in the first measurement layer of the forward region, where the background is highest. The muon trigger system covers the range $|\eta|<2.4$ with resistive-plate chambers in the barrel, and thin-gap chambers in the endcap regions.

A two-level trigger system [20] is used to select events for offline analysis. The first-level trigger is implemented in hardware and uses a subset of the detector information. This is followed by the software-based high-level trigger, reducing the event rate to about $1 \mathrm{kHz}$.

\section{Data and simulation}

This measurement utilises data collected by the ATLAS detector during the 2015 and 2016 data-taking periods. The data were recorded with a combination of single-lepton triggers, picking up events containing either an isolated lepton above a low- $p_{\mathrm{T}}$ threshold or a high$p_{\mathrm{T}}$ lepton without any isolation requirement. The lower $p_{\mathrm{T}}$ threshold for the isolated electron (muon) trigger ranges from 24 (20) to $26 \mathrm{GeV}$ depending on the instantaneous luminosity. The higher $p_{\mathrm{T}}$ threshold is $50(60) \mathrm{GeV}$ for the electron (muon) case over all the data-taking periods. Signal events satisfying the event selection described in section 4 are expected to have an overall trigger efficiency of $98 \%$.

Monte Carlo event simulation was deployed to model the signal and various background processes (summarised in table 1). In the determination of integrated cross-sections, the $A_{Z Z}$ and $C_{Z Z}$ factors as well as the $E_{\mathrm{T}}^{\text {miss }}$ shape for the $Z Z$ signal process were obtained from simulation. The background contributions were either predicted by simulation or estimated in data with the assistance of simulation.

The $q q Z Z$ process was modelled with PowHEG-Box v2 [21-24] interfaced to PYTHIA8.186 [25] for modelling of the parton showering, hadronisation and underlying event (UEPS). The NLO matrix-element (ME) calculation set both the factorisation $\left(\mu_{\mathrm{F}}\right)$ and renormalisation $\left(\mu_{\mathrm{R}}\right)$ scales to the invariant mass of the $Z Z$ system $\left(m_{Z Z}\right)$, and used the NLO CT10 [26] parton distribution function (PDF). The UEPS algorithm used a set of tuned parameters called the AZNLO tune [27]. The production cross-sections as a function of $m_{Z Z}$ were corrected to NNLO QCD and NLO EW accuracies in the total phase space. The QCD $K$-factors were derived using the MATRIX program [13], which computes the NNLO cross-section using the same QCD scales and the NNLO CT10 PDF [28]. The EW correction was applied using $K$-factors provided by the authors of ref. [15]. The QCD correction is about $+10 \%$ for the entire $m_{Z Z}$ spectrum, while the EW correction 


\begin{tabular}{|c|c|c|c|}
\hline Process & Generator & Simulation accuracy & Cross-section accuracy \\
\hline \multirow{2}{*}{$q q Z Z$} & Powheg-Box v2 + Pythia8.186 & NLO QCD & NNLO QCD + NLO EW \\
\hline & SHERPA 2.2 .2 & \multicolumn{2}{|c|}{ NLO QCD 0-1p, LO QCD 2-3p } \\
\hline \multirow{2}{*}{$g g Z Z$} & GG2VV3.1.6 + PYTHIA8.186 & LO QCD & NLO QCD \\
\hline & SHERPA2.1.1 & \multicolumn{2}{|c|}{ LO QCD $0-1 p$} \\
\hline$q q Z Z$ (aTGCs) & SHERPA2.1.1 & \multicolumn{2}{|c|}{ NLO QCD 0-1p, LO QCD 2-3p } \\
\hline \multirow{2}{*}{$W Z$} & Powheg-Box v2 + Pythia8.186 & \multirow{2}{*}{\multicolumn{2}{|c|}{ NLO QCD }} \\
\hline & Powheg-Box v2 + HeRwig ++ & & \\
\hline$W W$ & Powheg-Box v2 + Pythia 8.186 & \multicolumn{2}{|c|}{ NLO QCD } \\
\hline$q q Z Z \rightarrow 4 \ell$ & Powheg-Box v2 + Pythia8.186 & NLO QCD & NNLO QCD + NLO EW \\
\hline$g g Z Z \rightarrow 4 \ell$ & GG2VV3.1.6 + PYTHIA8.186 & LO QCD & NLO QCD \\
\hline$Z+$ jets & SHERPA 2.2 .1 & NLO QCD 0-2p, LO QCD 3-5p & NNLO QCD \\
\hline $\mathrm{t} \overline{\mathrm{t}}$ & Powheg-Box v2 + Pythia6.428 & NLO QCD & NNLO QCD \\
\hline$W t$ & Powheg-Box v2 + Pyтhia6.428 & NLO QCD & NNLO QCD \\
\hline$V V V$ & SHERPA2.1.1 & \multicolumn{2}{|c|}{ NLO QCD } \\
\hline$t \bar{t} V$ & MADGRAPH5_AMC@NLO + PYTHIA8.186 & LO QCD & NLO QCD \\
\hline
\end{tabular}

Table 1. Summary of Monte Carlo event simulation tools with their theoretical accuracy for each process, where "p" stands for parton(s). For the first two signal processes and the $W Z$ process, the first (second) row describes the baseline (alternative) simulation. The theoretical accuracy of the normalisation used for the total production cross-section of each process is shown in the last column.

is about $-4 \%$ at low $m_{Z Z}$ but has a larger impact at high $m_{Z Z}$, which cancels out the positive QCD correction for $m_{Z Z}$ around $500 \mathrm{GeV}$. An alternative sample was generated with SHERPA2.2.2 [29] using the NNLO NNPDF3.0 PDF [30] and the same choice of QCD scales. The SHERPA generator and its associated UEPS algorithm has NLO QCD accuracy for inclusive observables and extended QCD precision for events with one or more outgoing partons (NLO for up to one parton, LO for two and three partons).

The $g g Z Z$ events were simulated with the LO GG2Vv3.1.6 [31, 32] generator using the NNLO CT10 PDF, and then interfaced to Pythia8.186 using the A14 tune [33]. The production cross-section was corrected to NLO QCD accuracy using a $K$-factor of 1.7 reported in ref. [34]. An alternative modelling was provided by SHERPA2.1.1 [35] with the NLO CT10 PDF, which extended the LO QCD calculation to events with one parton. Both generators used $m_{Z Z} / 2$ for the QCD scales, and they incorporated both the fermion-loop and the Higgs processes, together with the interference between the two.

To study the effects of aTGCs, an additional sample for the SM $q q Z Z$ process was generated at NLO in QCD using ShERPA2.1.1 with the NLO CT10 PDF. The simulated sample was interfaced to a parton-level program [1] following the procedures detailed in ref. [36], and then event-by-event weights reflecting the relative change in the cross-sections due to any aTGCs were computed. A parameterisation of aTGC contributions as a function of any kinematic variable can be derived with this information. This procedure was adopted in the previous $Z Z$ measurements [6-8].

Production of $Z Z \rightarrow 4 \ell$ events was modelled in the same way as the signal events. The diboson background processes $W Z$ and $W W$ were generated with PowHEG-Box v2 using the NLO ME calculation and the NLO CT10 PDF, and then interfaced to PYTHIA8.186 
with the AZNLO tune. An alternative $W Z$ sample was produced with PowHEG-Box v2 interfaced to HERWIG++ [37], for the study of UEPS uncertainties. The interference between the $W W$ and $Z Z$ processes in the $\ell \ell \nu \nu$ final state was found to be negligible [16] and was therefore not considered in this analysis. Both the t $\overline{\mathrm{t}}$ and $W t$ events were simulated at NLO in QCD with Powheg-Box v2 [38, 39] and interfaced to Pythia6.428 [40], and the production cross-sections were corrected to NNLO QCD precision [41, 42]. ShERPA2.2.1 with the NNLO NNPDF3.0 PDF was used to model the $Z+$ jets process. The production cross-section for the $Z+$ jets process was calculated with NNLO QCD precision, while the simulation has NLO QCD precision for events with zero, one and two partons, and provided a LO QCD description for events with three to five partons. The rare $V V V$ background, consisting of $W W W, W W Z, W Z Z$ and $Z Z Z$ processes, was modelled with SHERPA2.1.1 with NLO QCD precision. MADGraPh5_AMC@NLO [43] interfaced to Pythia8.186 was used to generate the $t \bar{t} V$ background events that account for $\mathrm{t} \overline{\mathrm{t}} W, \mathrm{t} \overline{\mathrm{t}} Z$ and $\mathrm{t} \overline{\mathrm{t}} W W$ production processes. The $t \bar{t} V$ process was calculated at LO QCD accuracy, and its production cross-section was corrected to NLO QCD precision [43].

Generated events were then processed through the ATLAS detector simulation [44] based on GEANT4 [45] to emulate the response of the detector to the final-state particles. Pile-up was simulated with PYTHIA8.186 using the A2 tune [46] and overlaid on simulated events to mimic the real collision environment. The distribution of the average number of interactions per bunch crossing in the simulation was weighted to reflect that in data. Simulated events were processed with the same reconstruction algorithms as for the data. Furthermore, the lepton momentum scale and resolution, and the lepton reconstruction, identification, isolation and trigger efficiencies in the simulation were corrected to match those measured in data.

\section{Selection of $\ell \ell \nu \nu$ events}

This analysis selects a detector signature with a pair of high- $p_{\mathrm{T}}$ isolated electrons $(e e)$ or muons $(\mu \mu)$ and large $E_{\mathrm{T}}^{\text {miss }}$. The $e e$ and $\mu \mu$ channels are combined to obtain the final results. The event selection strategy was optimised to cope with the large background contamination. The selection requirements lead to a highly boosted $Z$ boson back-to-back with the missing transverse momentum vector $\left(\vec{E}_{\mathrm{T}}^{\text {miss }}\right)$. Backgrounds are further reduced by removing events with extra leptons or any jets containing $b$-hadrons (" $b$-jets"). Therefore, a precise understanding of the overall reconstruction and selection of leptons, jets, and $E_{\mathrm{T}}^{\text {miss }}$ is required in this measurement.

Events are first required to have a collision vertex associated with at least two tracks each with $p_{\mathrm{T}}>0.4 \mathrm{GeV}$. The vertex with the highest scalar $p_{\mathrm{T}}^{2}$ sum of the associated tracks is referred to as the primary vertex.

Electrons are reconstructed from energy deposits in the EM calorimeter matched to a track reconstructed in the ID. The electron identification imposes selections on the number of hits in the ID and requirements on a likelihood discriminant, built from variables related to EM calorimeter shower shapes, track-cluster matching, track quality, and transition radiation [47]. Electrons must satisfy the "medium" identification criterion [47], which is about 
$90 \%$ efficient for electrons with $p_{\mathrm{T}} \approx 40 \mathrm{GeV}$. Candidate electrons must have $p_{\mathrm{T}}>7 \mathrm{GeV}$ and pseudorapidity $|\eta|<2.47$. Muons are reconstructed by combining all the hits associated with a pair of matched tracks reconstructed in the ID and MS, taking into account the energy loss in the calorimeter. Muons are identified by requiring a sufficient number of ID and MS hits, and good consistency between the ID and MS track measurements as well as good combined fit quality [48], and they must satisfy the "medium" identification criterion [48], which has an overall efficiency of $96 \%$. Candidate muons are required to have $p_{\mathrm{T}}>7 \mathrm{GeV}$ and $|\eta|<2.5$. To further suppress misidentified lepton and cosmic-ray background contributions, the absolute value of the longitudinal impact parameter of leptons with respect to the primary vertex must be smaller than $0.5 \mathrm{~mm}$, and the transverse impact parameter divided by its error must be less than 5 (3) for electrons (muons). In addition, the "loose" isolation criteria defined in refs. [47, 48] are applied. The isolation selection imposes requirements on both the track-based and calorimeter-based isolation variables, and varies as a function of $p_{\mathrm{T}}$ to maintain a uniform efficiency above $98 \%$ for prompt leptons.

Jets are reconstructed with the anti- $k_{t}$ algorithm [49] with radius parameter $R=0.4$, using as input positive-energy topological clusters in the calorimeters [50-52]. The jet energy scale is calibrated using simulation and further corrected with in situ methods [51]. Candidate jets must have $p_{\mathrm{T}}>20 \mathrm{GeV}$ and $|\eta|<4.5$. Additional requirements using the track and vertex information inside a jet [53] are applied for jets with $p_{\mathrm{T}}<60 \mathrm{GeV}$ and $|\eta|<2.5$ to suppress pile-up contributions. Candidate $b$-jets $\left(p_{\mathrm{T}}>20 \mathrm{GeV}\right.$ and $\left.|\eta|<2.5\right)$ are identified with an algorithm providing $85 \%$ signal efficiency and a rejection factor of 33 against light-flavour jets [54].

Leptons and jets may be close to each other or overlapping, even after implementing the full set of object selections. The appearance of such overlapping objects may lead to ambiguities in the event selection and in the energy measurement of the physics objects. A common procedure in the ATLAS experiment, as detailed in ref. [55], is applied to resolve the ambiguities. This requirement helps to suppress the occurrence of two "problematic" scenarios, one with energy measurement of electrons biased due to nearby jets, and the other with a jet producing non-prompt muons through meson decays in flight.

The $\vec{E}_{\mathrm{T}}^{\text {miss }}$ vector is computed as the negative of the vector sum of transverse momenta of all the leptons and jets, as well as the tracks originating from the primary vertex but not associated with any of the leptons or jets ("soft-term") [56]. The soft-term is computed in a way minimising the impact of pile-up in the $E_{\mathrm{T}}^{\text {miss }}$ reconstruction.

Candidate events are preselected by requiring exactly two selected electrons or muons with opposite charges and $p_{\mathrm{T}}>20 \mathrm{GeV}$. The leading lepton is further required to have $p_{\mathrm{T}}>30 \mathrm{GeV}$, well above the threshold of the single-lepton triggers. To suppress the $W Z$ background, events containing any additional lepton satisfying the "loose" rather than "medium" identification requirement, in addition to the other requirements, are rejected. The dilepton invariant mass $\left(m_{\ell \ell}\right)$ is required to be in the range between 76 and $106 \mathrm{GeV}$, which largely reduces the contamination from the non-resonant- $\ell \ell$ background. Figure 2 shows the observed and expected $E_{\mathrm{T}}^{\text {miss }}$ spectra after imposing the above requirements ("preselection"). The fractional experimental uncertainties in the expected spectra increase 

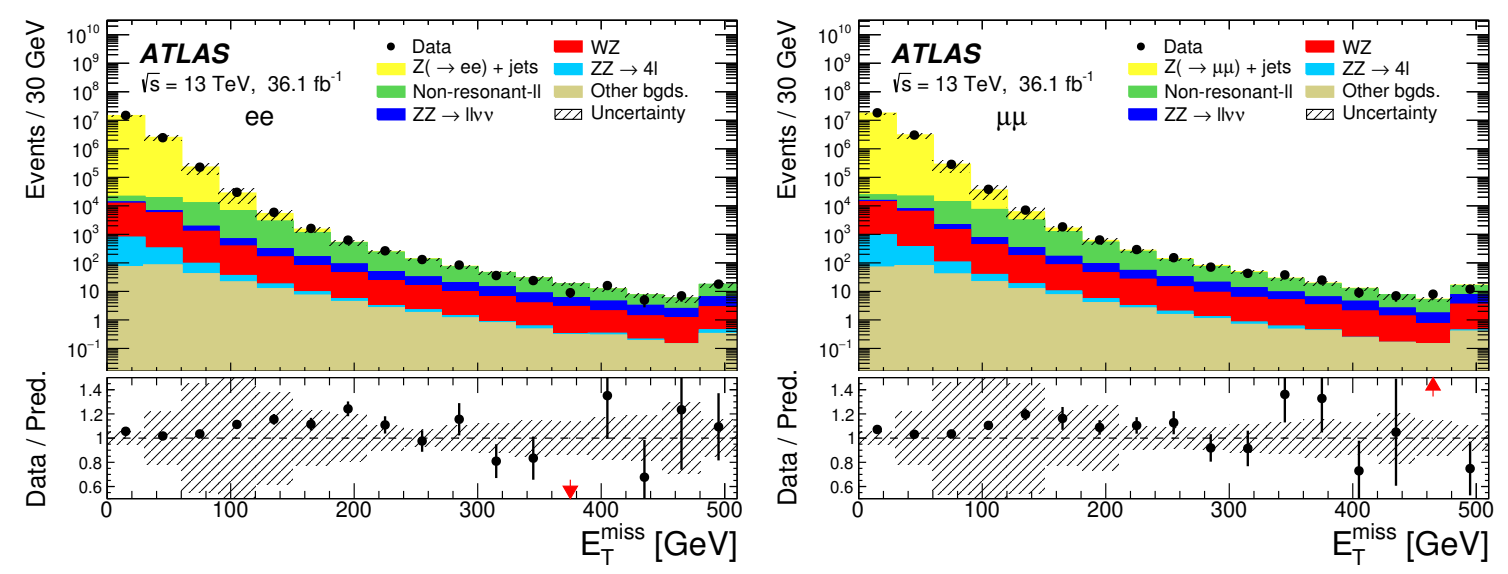

Figure 2. The $E_{\mathrm{T}}^{\text {miss }}$ distributions after the preselection for the ee (left) and $\mu \mu$ (right) channels. The expectation is derived from simulation and the hashed band accounts for the statistical and experimental uncertainties. The experimental uncertainties are described in section 7 . The last bin in the distributions contains the overflow events. The arrows indicate that the differences between the data and the expectation in some bins exceed the $y$-axis scope of the bottom plots. The "other" background corresponds to the $V V V$ and $t \bar{t} V$ processes.

as a function of $E_{\mathrm{T}}^{\text {miss }}$ in the region dominated by the $Z+$ jets process, as a result of the asymmetric migration effects along the steeply falling $E_{\mathrm{T}}^{\mathrm{miss}}$ distribution and the large jet-related uncertainty for $Z+$ jets events at high $E_{\mathrm{T}}^{\text {miss }}$. The top-quark processes with genuine $E_{\mathrm{T}}^{\text {miss }}$ dominate the high $E_{\mathrm{T}}^{\text {miss }}$ region. For $E_{\mathrm{T}}^{\text {miss }}$ around $200 \mathrm{GeV}$, top-quark events generally contain less jet activity than $Z+$ jets events: this leads to correspondingly smaller experimental uncertainties. The experimental uncertainties are elaborated in section 7 . The data sample after the preselection is dominated by the $Z+$ jets and non-resonant- $\ell \ell$ processes. To suppress these backgrounds, a further selection based on $E_{\mathrm{T}}^{\text {miss }}$ and event topology is applied.

Candidate events are required to have $E_{\mathrm{T}}^{\text {miss }}>110 \mathrm{GeV}$ and $V_{\mathrm{T}} / S_{\mathrm{T}}>0.65$, where $V_{\mathrm{T}}$ is the magnitude of the vector sum of transverse momenta of selected leptons and jets, and $S_{\mathrm{T}}$ is the scalar $p_{\mathrm{T}}$ sum of the corresponding objects. The variable $V_{\mathrm{T}} / S_{\mathrm{T}}$ was found to be less sensitive to jet experimental uncertainties than similar variables such as $E_{\mathrm{T}}^{\mathrm{miss}} / S_{\mathrm{T}}$. To further reduce the impact of jet energy scale uncertainties, the calculation of $V_{\mathrm{T}}$ and $S_{\mathrm{T}}$ uses "hard jets" which are required to have $p_{\mathrm{T}}>25 \mathrm{GeV}$ for the central region $(|\eta|<2.4)$ and $p_{\mathrm{T}}>40 \mathrm{GeV}$ for the forward region $(2.4<|\eta|<4.5)$. The $E_{\mathrm{T}}^{\text {miss }}$ cut suppresses the $Z+$ jets contamination by many orders of magnitude, and the residual $Z+$ jets events, which have large fake $E_{\mathrm{T}}^{\text {miss }}$, are further suppressed by the $V_{\mathrm{T}} / S_{\mathrm{T}}$ requirement. As the consequence of the combined $E_{\mathrm{T}}^{\text {miss }}$ and $V_{\mathrm{T}} / S_{\mathrm{T}}$ requirement, the $Z+$ jets process only constitutes a small fraction of the total background after the full selection.

Additional selection criteria based on angular variables are imposed to ensure the desired detector signature, which helps to further reject the $Z+$ jets and non-resonant$\ell \ell$ background events. The azimuthal angle difference between the dilepton system and $\vec{E}_{\mathrm{T}}^{\text {miss }}, \Delta \phi\left(\vec{p}_{\mathrm{T}}^{\ell \ell}, \vec{E}_{\mathrm{T}}^{\text {miss }}\right)$, must be larger than 2.2 radians, and the selected leptons must be close to each other, with the distance $\Delta R_{\ell \ell}=\sqrt{\left(\Delta \phi_{\ell \ell}\right)^{2}+\left(\Delta \eta_{\ell \ell}\right)^{2}}<1.9$. Finally, events 


\begin{tabular}{|lc|}
\hline Step & Selection criteria \\
\hline Two leptons & Two opposite-sign leptons, leading (subleading) $p_{\mathrm{T}}>30(20) \mathrm{GeV}$ \\
\hline Jets & $p_{\mathrm{T}}>20 \mathrm{GeV},|\eta|<4.5$, and $\Delta R>0.4$ relative to the leptons \\
\hline Third-lepton veto & No additional lepton with $p_{\mathrm{T}}>7 \mathrm{GeV}$ \\
\hline$m_{\ell \ell}$ & $76<m_{\ell \ell}<106 \mathrm{GeV}$ \\
\hline Hard jets & $p_{\mathrm{T}}>25 \mathrm{GeV}$ for $|\eta|<2.4, p_{\mathrm{T}}>40 \mathrm{GeV}$ for $2.4<|\eta|<4.5$ \\
\hline$E_{\mathrm{T}}^{\text {miss }}$ and $V_{\mathrm{T}} / S_{\mathrm{T}}$ & $E_{\mathrm{T}}^{\text {miss }}>110 \mathrm{GeV}$ and $V_{\mathrm{T}} / S_{\mathrm{T}}>0.65$ \\
\hline$\Delta R_{\ell \ell}$ & $\Delta R_{\ell \ell}<1.9$ \\
\hline$\Delta \phi\left(\vec{p}_{\mathrm{T}}^{\ell \ell}, \vec{E}_{\mathrm{T}}^{\text {miss }}\right)$ & $\Delta \phi\left(\vec{p}_{\mathrm{T}}^{\ell \ell}, \vec{E}_{\mathrm{T}}^{\text {miss }}\right)>2.2$ radians \\
\hline$b$-jet veto & $N(b$-jets $)=0$ with $b$-jet $p_{\mathrm{T}}>20 \mathrm{GeV}$ and $|\eta|<2.5$ \\
\hline
\end{tabular}

Table 2. Event selection criteria for the $\ell \ell \nu \nu$ signature.

\begin{tabular}{|l|l|}
\hline Total phase space & Born-level leptons $(e e$ or $\mu \mu)$ \\
& $66<m_{\ell \ell}, m_{\nu \nu}<116 \mathrm{GeV}$ \\
\hline & Dressed leptons $(e$ or $\mu): p_{\mathrm{T}}>7 \mathrm{GeV},|\eta|<2.5$ \\
& Jets: $p_{\mathrm{T}}>20 \mathrm{GeV},|\eta|<4.5$ \\
Fiducial phase space & Reject leptons if overlapping with a jet within $\Delta R<0.4$ \\
& Two leptons with leading (subleading) $p_{\mathrm{T}}>30(20) \mathrm{GeV}$ \\
& $76<m_{\ell \ell}<106 \mathrm{GeV}$ \\
& $E_{\mathrm{T}}^{\text {miss }}>90 \mathrm{GeV}$ and $V_{\mathrm{T}} / S_{\mathrm{T}}>0.65$ \\
& $\Delta \phi\left(\vec{p}_{\mathrm{T}}^{\ell \ell}, \vec{E}_{\mathrm{T}}^{\text {miss }}\right)>2.2$ radians and $\Delta R_{\ell \ell}<1.9$ \\
\hline
\end{tabular}

Table 3. Definitions of the total and fiducial phase spaces for the $Z Z \rightarrow \ell \ell \nu \nu$ signal.

containing one or more $b$-jets are vetoed to further suppress the t $\bar{t}$ and $W t$ backgrounds. The full event selection is summarised in table 2 . Figure 3 gives the observed and simulated spectra for $V_{\mathrm{T}} / S_{\mathrm{T}}, \Delta R_{\ell \ell}, \Delta \phi\left(\vec{p}_{\mathrm{T}}^{\ell \ell}, \vec{E}_{\mathrm{T}}^{\text {miss }}\right)$, and the number of $b$-jets, where each plot is made with the implementation of all the cuts prior to the cut on that variable, according to the cut sequence in table 2 .

\section{$5 \quad$ Total and fiducial phase spaces}

The definitions of the total and fiducial phase spaces are summarised in table 3 . The total phase space is defined as in ref. [8] for the $Z Z \rightarrow 4 \ell$ measurement, requiring $66<$ $m_{\ell \ell}, m_{\nu \nu}<116 \mathrm{GeV}(\ell=e$ or $\mu)$, where the leptons and neutrinos originate from the $Z$-boson decays. The four-momenta of the leptons are defined at Born level, i.e. before any QED final-state radiation.

The fiducial phase space is defined with a set of criteria very close to that of the detector-level event selection (table 2). This strategy helps to reduce the amount of phase- 

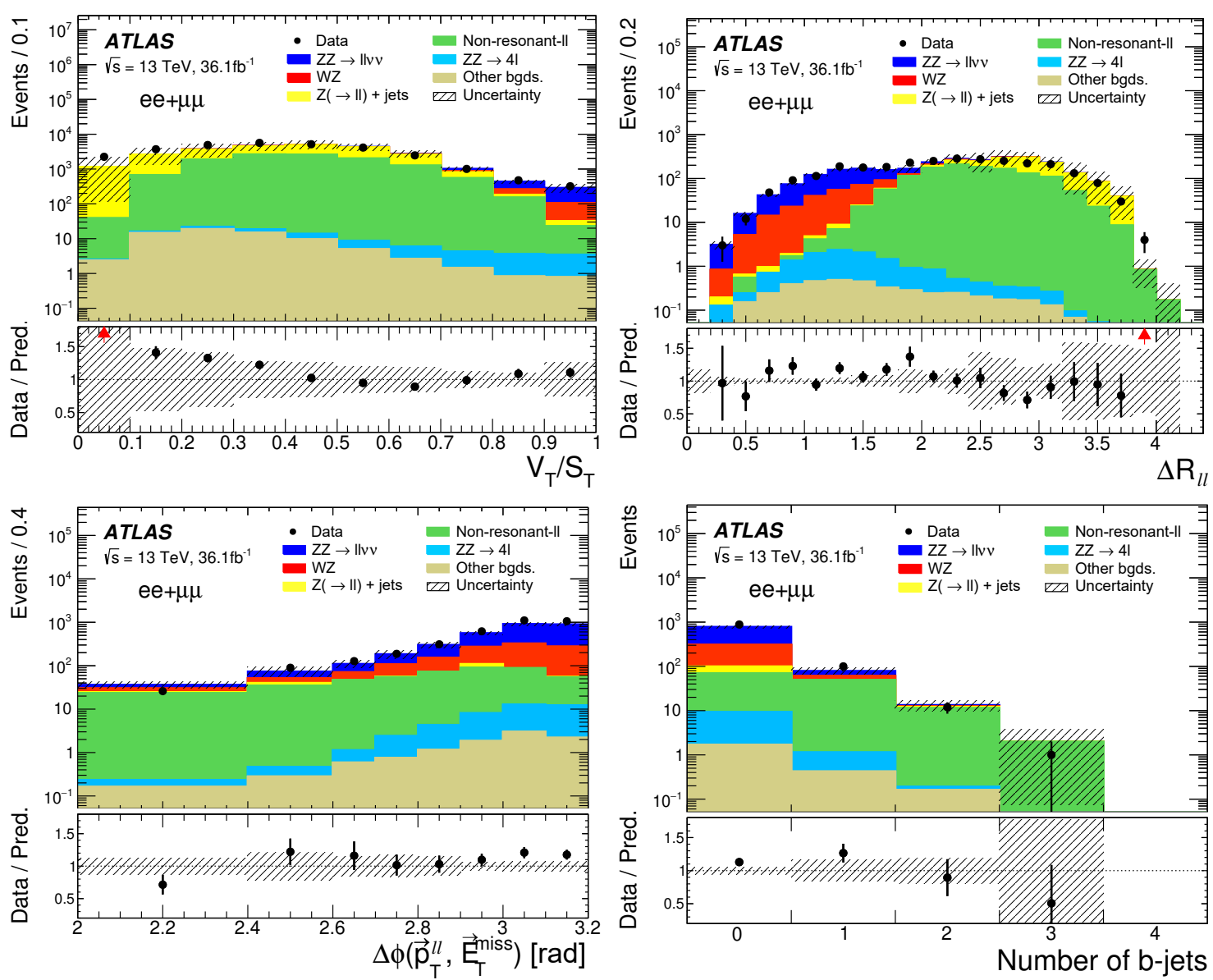

Figure 3. The $V_{\mathrm{T}} / S_{\mathrm{T}}, \Delta R_{\ell \ell}, \Delta \phi\left(\vec{p}_{\mathrm{T}}^{\ell \ell}, \vec{E}_{\mathrm{T}}^{\text {miss }}\right)$, and the number of $b$-jets distributions for the combination of $e e$ and $\mu \mu$ channels with the implementation of all the cuts in table 2 prior to the cut on that variable. The expectation is derived from simulation and the hashed band accounts for the statistical and experimental uncertainties. The first bin in the distribution of $\Delta \phi\left(\vec{p}_{\mathrm{T}}^{\ell \ell}, \vec{E}_{\mathrm{T}}^{\text {miss }}\right)$ (bottom left) contains the underflow events. The arrow in the $V_{\mathrm{T}} / S_{\mathrm{T}}$ distribution indicates that the difference between the data and the expectation exceeds the $y$-axis scope of the bottom plot. The "other" background corresponds to the $V V V$ and $t \bar{t} V$ processes.

space extrapolation in the fiducial measurements and therefore minimises the theoretical uncertainties of the results. The criteria are applied to "particle-level" physics objects, which are reconstructed from stable final-state particles, prior to their interactions with the detector. For electrons and muons, QED final-state radiation is partly recovered by adding to the lepton four-momentum the four-momenta of surrounding photons not originating from hadrons within an angular distance $\Delta R<0.1$ (dressed leptons). Particle-level jets are built with the anti- $k_{t}$ algorithm with radius parameter $R=0.4$, using all finalstate particles as the input (excluding muons and neutrinos). As shown in table 3 , the selection requirements for the fiducial phase space closely follow those in table 2 . The $\vec{E}_{\mathrm{T}}^{\text {miss }}$ vector is defined as the sum of transverse momenta of the two neutrinos from the $Z$-boson decays. This measurement requires large $E_{\mathrm{T}}^{\text {miss }}$, which has a detector resolution of around $10 \mathrm{GeV}$ [56] in the phase space considered here. To accommodate the majority of 


\begin{tabular}{|c|c|c|c|c|c|c|}
\hline & \multicolumn{2}{|c|}{$Z Z$} & \multicolumn{2}{|c|}{$q q Z Z$} & \multicolumn{2}{|c|}{$g g Z Z$} \\
\hline & $e e$ & $\mu \mu$ & $e e$ & $\mu \mu$ & $e e$ & $\mu \mu$ \\
\hline Signal yield & $\begin{array}{c}220 \pm 15 \\
\left( \pm 2_{\text {stat }} \pm 7 \text { exp }\right. \\
\left. \pm 13_{\text {theory }}\right)\end{array}$ & $\begin{array}{c}229 \pm 15 \\
\left( \pm 2_{\text {stat }} \pm 7 \text { exp }\right. \\
\left. \pm 13_{\text {theory }}\right) \\
\end{array}$ & $194 \pm 12$ & $202 \pm 12$ & $25 \pm 15$ & $26 \pm 16$ \\
\hline $\begin{array}{l}C_{Z Z} \\
\sigma_{Z Z \rightarrow \ell \ell \nu \nu}^{\text {exp,fid }} \\
A_{Z Z} \\
\sigma_{Z Z}^{\text {exp,tot }}\end{array}$ & $\begin{array}{r}22.4 \\
15.7\end{array}$ & $1.3 \mathrm{fb}$ & $\begin{array}{r}(54.7 \pm 1.7) \% \\
18.8 \pm \\
(5.3 \pm \\
13.9 \pm\end{array}$ & $\begin{array}{l}(56.6 \pm 1.7) \% \\
1.0 \mathrm{fb} \\
0.1) \% \\
0.4 \mathrm{pb}\end{array}$ & $\begin{array}{r}(53.1 \pm 1.8) \% \\
2.6 \pm \\
(5.3 \pm \\
1.8 \pm\end{array}$ & $\begin{array}{l}(55.5 \pm 2.2) \% \\
0.8 \mathrm{fb} \\
0.3) \% \\
.6 \mathrm{pb}\end{array}$ \\
\hline
\end{tabular}

Table 4. Predictions for the signal yields at detector level, for the $C_{Z Z}$ and $A_{Z Z}$ coefficients defined in eq. (1.1), and for the cross-sections in the fiducial and total phase spaces. The first column gives the corresponding predictions for the total $Z Z$ process, combined from those shown separately for the $q q Z Z$ and $g g Z Z$ sub-processes. The errors include both the statistical and systematic uncertainties (see section 7). The statistical, experimental, and theoretical uncertainties are also shown separately for the combined signal yields.

the events selected at detector level, the $E_{\mathrm{T}}^{\text {miss }}$ threshold is therefore lowered to $90 \mathrm{GeV}$ in the fiducial region. The efficiency of the $b$-jet veto is found to be $98 \%$ in the fiducial region and consistent between the Powheg+Pythia 8 and Sherpa generators. No requirement is made on the number of $b$-jets in the fiducial selection.

Table 4 gives the expected signal yields at detector level, the $A_{Z Z}$ and $C_{Z Z}$ factors, and the predicted cross-sections. The $q q Z Z$ and $g g Z Z$ processes have similar final-state kinematic distributions and their $A_{Z Z}$ and $C_{Z Z}$ factors are similar. The corresponding factors for the total $Z Z$ process are averaged from that for the two sub-processes, weighted by the respective cross-sections. The cross-section predictions for the total phase space are corrected for the branching fraction of the $Z Z \rightarrow \ell \ell \nu \nu$ decays, $1.35 \%$ with a negligible uncertainty, obtained from refs. [57, 58]. The expected fiducial and total cross-sections, $\sigma_{Z Z \rightarrow \ell \ell \nu \nu}^{\text {exp,fid }}$ and $\sigma_{Z Z}^{\text {exp,tot }}$, are calculated from simulation, including the higher-order corrections detailed in section 3. The total uncertainties in these predictions are also provided in table 4, and the procedures used to derive these uncertainties are described in section 7 .

\section{Background estimation}

After the event selection, the overall signal-to-background ratio is about 1.7. The $W Z$ and non-resonant- $\ell \ell$ backgrounds account for $72 \%$ and $21 \%$ of the total background contribution, respectively, and are estimated from control regions in data. The $Z+$ jets background is largely suppressed, yielding a relative contribution of only $4 \%$, and is estimated from data. Finally, the small remaining contributions from other processes, amounting in total to $3 \%$ of the total background, are estimated from simulation. The various background estimates and their uncertainties are described below.

To estimate the dominant resonant background from $W Z$ production, a control region enriched in $W Z$ events, with a purity of $90 \%$, is defined using the preselection criteria, except that a third lepton with $p_{\mathrm{T}}>20 \mathrm{GeV}$ and satisfying the medium identification criteria is required. Several further selections such as $V_{\mathrm{T}} / S_{\mathrm{T}}>0.3, b$-jets veto, and $m_{\mathrm{T}}^{W}>60 \mathrm{GeV}$, 
where $m_{\mathrm{T}}^{W}$ is constructed from the third lepton's transverse momentum and the $\vec{E}_{\mathrm{T}}^{\text {miss }}$ vector, ${ }^{3}$ are applied to suppress non- $W Z$ contributions. A normalisation factor $\left(f_{W Z}\right)$ is calculated in the control region as the number of observed events in data, subtracting the non- $W Z$ contributions estimated from simulation, divided by the predicted $W Z$ yield. The factor $f_{W Z}$ is found to be $1.26 \pm 0.04$ (stat), which is consistent with a recent $W Z$ measurement [59], performed within a broader fiducial phase space and using a recent calculation of the $W Z$ total cross-section at NNLO in QCD [60,76]. The statistical uncertainty of the data in the control region leads to a $3 \%$ uncertainty in the $W Z$ estimate in the signal region. The systematic uncertainty is evaluated for the ratio of the $W Z$ predictions in the signal and control regions. The experimental uncertainty in this ratio is $3.5 \%$, and the theoretical uncertainty is $3.3 \%$, calculated as the sum in quadrature of the PDF, scale, and UEPS uncertainties. The non- $W Z$ contribution in the control region is less than $10 \%$. The uncertainty related to the subtraction of the non- $W Z$ contribution, estimated by imposing cross-section uncertainties for all the relevant processes, is found to be about $2 \%$. The total uncertainty in the $W Z$ estimate is about $6 \%$. The kinematic distributions are estimated from simulation, with both the experimental and theoretical uncertainties considered.

To estimate the non-resonant- $\ell \ell$ background, including $W W$, top-quark ( $\mathrm{t} \overline{\mathrm{t}}$ and $W t$ ), and $Z \rightarrow \tau \tau$ production, a control region dominated by the non-resonant- $\ell$ processes (with a purity above $95 \%$ ) is defined with all the event selection criteria in table 2 , except that the final state is required to contain an opposite-sign $e \mu$ pair. The non-resonant- $\ell \ell$ contribution in the $e e(\mu \mu)$ channel is calculated as one half of the observed data yield after subtracting the contribution from the other background processes in the control region, and then corrected for the difference in the lepton reconstruction and identification efficiencies between selecting an $e \mu$ pair and an $e e(\mu \mu)$ pair. The lepton efficiency correction is derived as the square root of the ratio of the numbers of $\mu \mu$ and $e e$ events in data after the preselection. The choice of deriving the correction after preselection minimises the resulting statistical uncertainty. The total uncertainty in the non-resonant- $\ell \ell$ estimate is about $16 \%$, including the statistical uncertainty of the data in the control region (14\%) and the method bias estimated from simulation $(7 \%)$. The kinematic distributions for the nonresonant- $\ell \ell$ background estimate in the signal region are predicted with simulation, and the assigned systematic uncertainty covers the experimental uncertainty in the simulated shape as well as the difference between data and simulation in the control region.

Figure 4 gives two examples of comparing data and predictions in the $W Z$ and nonresonant- $\ell \ell$ background control regions. The left-hand figure is the $m_{\mathrm{T}}^{W}$ distribution in the $W Z$ control region, where the normalisation factor $f_{W Z}$ is applied to the $W Z$ simulation and good agreement between the observed and predicted shapes is found. The righthand figure is the $E_{\mathrm{T}}^{\text {miss }}$ distribution in the non-resonant- $\ell \ell$ control region, where the $W W$ and top-quark ( $\mathrm{t} \overline{\mathrm{t}}$ and $W t$ ) production processes are dominant. Both the statistical and experimental uncertainties are included in the hashed bands in these figures.

A data-driven method is used to estimate the $Z+$ jets background. This method defines three independent regions (labelled as $\mathrm{B}, \mathrm{C}$ and $\mathrm{D}$ ) which are enriched in $Z+$

${ }^{3} m_{\mathrm{T}}^{W}=\sqrt{2 p_{\mathrm{T}}^{\ell} E_{\mathrm{T}}^{\mathrm{miss}}\left[1-\cos \Delta \phi\left(\vec{p}_{\mathrm{T}}^{\ell}, \vec{E}_{\mathrm{T}}^{\text {miss }}\right)\right]}$. 

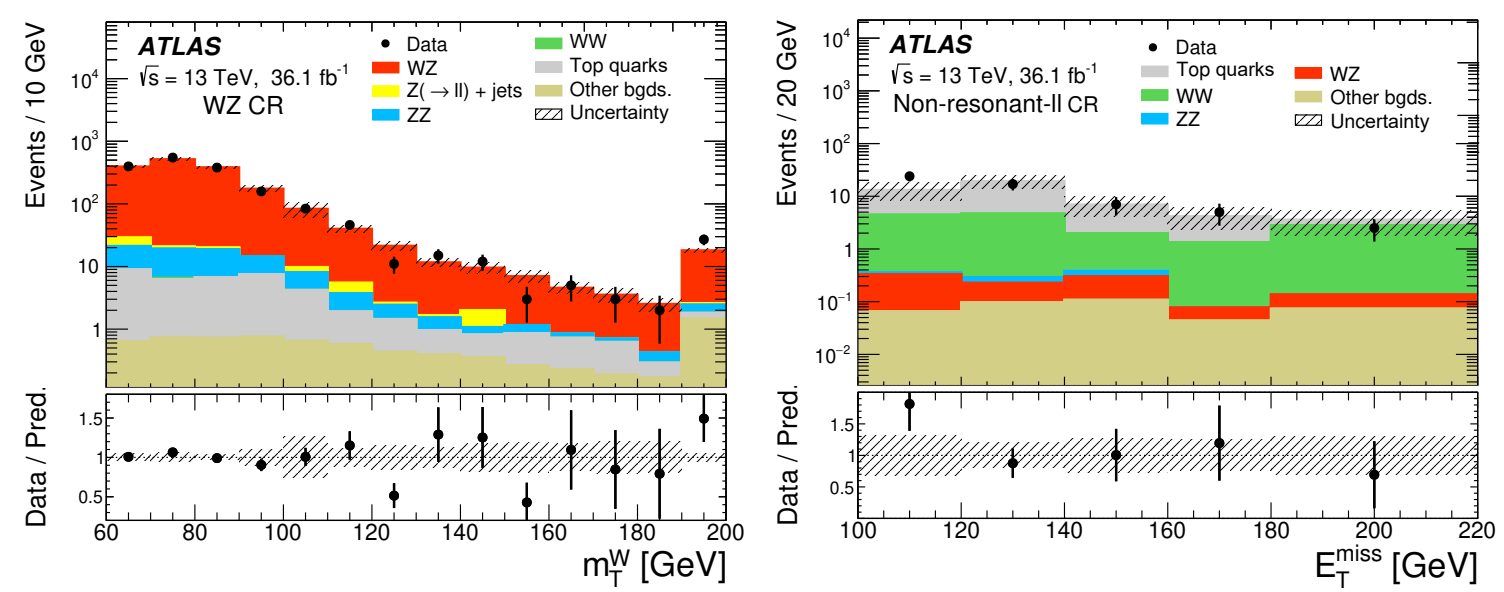

Figure 4. Distributions in the control regions $(\mathrm{CR})$, of $m_{\mathrm{T}}^{W}$ for the $W Z \mathrm{CR}$ (left) and of $E_{\mathrm{T}}^{\text {miss }}$ for the non-resonant- $\ell \ell \mathrm{CR}$ (right). The data are compared with the predictions from simulation, where the $W Z$ contribution is scaled by the normalisation factor of 1.26 described in the text. The last bin in the distributions contains the overflow events. The hashed bands include both the statistical and experimental uncertainties. The "other" background corresponds to the $V V V$ and $t \bar{t} V$ processes.

jets events and are not overlapping with the signal region (labelled as $\mathrm{A}$ ). The data yields after subtracting the non- $Z$ contributions in these regions $\left(n_{\mathrm{B}}, n_{\mathrm{C}}\right.$ and $\left.n_{\mathrm{D}}\right)$ are used to predict the $Z+$ jets contribution in the signal region $\left(n_{\mathrm{A}}\right)$, calculated as $n_{\mathrm{C}} \times n_{\mathrm{B}} / n_{\mathrm{D}}$. The main assumption of the method is that $n_{\mathrm{A}} / n_{\mathrm{C}}=n_{\mathrm{B}} / n_{\mathrm{D}}$. The control region definitions are optimised to ensure that this assumption is valid within uncertainties evaluated from simulation. The control regions are defined using the preselection requirements plus the $b$-jets veto. A further requirement of $E_{\mathrm{T}}^{\mathrm{miss}}>30 \mathrm{GeV}$ and $V_{\mathrm{T}} / S_{\mathrm{T}}>0.2$ is imposed to remove the low- $E_{\mathrm{T}}^{\text {miss }}$ phase space which is far away from the signal region. The $E_{\mathrm{T}}^{\text {miss }}$ and $V_{\mathrm{T}} / S_{\mathrm{T}}$ variables are expected to have a small correlation with the topological variables, so the various requirements to define the control regions are grouped together, such that the correlations between regions are minimised. Specifically, two Boolean variables are defined as, $\alpha=" E_{\mathrm{T}}^{\text {miss }}>110 \mathrm{GeV}$ and $V_{\mathrm{T}} / S_{\mathrm{T}}>0.65 "$ and $\beta=" \Delta \phi\left(\vec{p}_{\mathrm{T}}^{\ell \ell}, \vec{E}_{\mathrm{T}}^{\text {miss }}\right)>2.2$ radians and $\Delta R_{\ell \ell}<1.9 "$ ". The four regions are then defined as follows:

- Region A: $\alpha=$ TRUE and $\beta=$ TRUE

- Region B: $\alpha=$ FALSE and $\beta=$ TRUE

- Region C: $\alpha=$ TRUE and $\beta=$ FALSE

- Region D: $\alpha=$ FALSE and $\beta=$ FALSE

Regions $\mathrm{B}$ and $\mathrm{D}$ are dominated by the $Z+$ jets process (with a purity greater than $95 \%$ ), while its relative contribution in region $\mathrm{C}$ is only $70 \%$ because the $\mathrm{t} \overline{\mathrm{t}}$ contribution in this phase space region remains large. The derived $Z+$ jets contribution is corrected for the closure factor $\left(n_{\mathrm{A}} / n_{\mathrm{C}} \times n_{\mathrm{D}} / n_{\mathrm{B}}\right)$ estimated from simulation. This factor is found to be 
0.9 and has a relative uncertainty of $48 \%$, consisting of the statistical (40\%), experimental $(22 \%)$, and methodology uncertainties $(15 \%)$. The experimental uncertainty in the closure factor is dominated by jet energy scale and resolution. The methodology uncertainty covers the variations obtained by changing the $E_{\mathrm{T}}^{\text {miss }}$ and $V_{\mathrm{T}} / S_{\mathrm{T}}$ thresholds in the low$E_{\mathrm{T}}^{\text {miss }}$ removal requirement by $40 \%$. The $Z+$ jets estimation is also subject to the statistical uncertainty of the data $(5 \%)$ and the subtraction of non- $Z$ contributions in the control regions $(5 \%)$. The non- $Z$ subtraction uncertainty is driven by the modelling uncertainty for the main non- $Z$ process in region $\mathrm{C}$ ( $\mathrm{t} \overline{\mathrm{t}}$ production), which is about $10-20 \%$ for $E_{\mathrm{T}}^{\text {miss }}$ above $100 \mathrm{GeV}$ [61]. The total uncertainty on the $Z+$ jets estimate is about $50 \%$. The kinematic distributions for the $Z+$ jets background in the signal region are derived from the data in region $\mathrm{C}$, together with a systematic uncertainty assigned in a way similar to that described above for the non-resonant- $\ell \ell$ background.

The $Z Z \rightarrow 4 \ell, V V V$ and $t \bar{t} V(V)$ backgrounds are estimated from simulation, and their contributions have a total uncertainty of 10-20\%, including both the theoretical crosssection $[8,62,63]$ and the experimental uncertainties.

\section{Systematic uncertainties}

The measurement results and predictions are subject to theoretical and experimental uncertainties, as well as uncertainties related to the background estimation. The background uncertainties are explained in section 6 . The statistical uncertainties of the simulated samples for both the signal and background processes are also taken into account wherever applicable. The systematic uncertainty sources for the signal process are detailed below.

The theoretical uncertainties for the dominant $q q Z Z$ signal sub-process are estimated with the Powheg+Pythia8 generator, since only the total cross-section has been calculated to NNLO QCD and NLO EW accuracies. The theoretical uncertainties originate from the PDF choice, the missing higher-order QCD calculation, and the UEPS modelling. The PDF uncertainty is calculated as the $68 \%$ confidence-level eigenvector uncertainty [26] of the nominal PDF used in the simulation. The uncertainty due to the QCD calculation, also referred to as the "scale" uncertainty, covers the variations of predictions from changing the QCD renormalisation and factorisation scales. The QCD scales are varied independently by factors ranging from one half to two, which in total yields seven different scale choices including the nominal one. The UEPS uncertainty is taken as the difference in the predictions between the HERWIG ++ and the default showering programs. The fractional theoretical uncertainty in $A_{Z Z}$ for the $q q Z Z$ process is about $1.8 \%$, while the overall uncertainties in the cross-section predictions in the total and fiducial phase spaces are about $3 \%$ and $5 \%$, respectively. The SHERPA generator is used to cross-check the nominal predictions, and the $A_{Z Z}$ factors from POWHEg and SHERPA are consistent with each other within the uncertainty. The $C_{Z Z}$ predictions from the two generators are found to be consistent within the statistical uncertainty of $1 \%$, and in this measurement, the theoretical uncertainty in $C_{Z Z}$ is neglected.

The understanding of the $p_{T}^{\ell \ell}$ spectrum in the fiducial phase space is crucial for the study of aTGCs, and the predictions from the two generators differ by up to $10 \%$ for 
$p_{\mathrm{T}}^{\ell \ell}$ around $300 \mathrm{GeV}$, which is slightly above the theoretical uncertainty of the PowHEG prediction. The POWHEG prediction with the $K$-factors applied has better precision in terms of the EW calculation, while the SHERPA generator is expected to give a better description of $Z Z$ production with extra QCD radiation. Finally, an uncertainty is applied to the $p_{\mathrm{T}}^{\ell \ell}$ prediction, as the sum in quadrature of the theoretical uncertainty estimated with Powheg and the difference between Powheg and SHerpa, which is about $5 \%$ for $p_{\mathrm{T}}^{\ell \ell}$ around $150 \mathrm{GeV}$ and increases to about $11 \%$ for $p_{\mathrm{T}}^{\ell \ell}$ above $250 \mathrm{GeV}$.

A constant $30 \%$ uncertainty is assigned to the total $g g Z Z$ cross-section prediction, which covers the uncertainties concerning the NLO $K$-factor [34] and the potential missing higher-order contributions [64]. The $A_{Z Z}$ predictions for the $g g Z Z$ process from the GG2VV and Sherpa generators are found to be consistent, and the $A_{Z Z}$ uncertainty is estimated with SHERPA and found to be $4.6 \%$. The theoretical uncertainty in $C_{Z Z}$ is neglected for the $g g Z Z$ process.

The major experimental uncertainties originate from the luminosity uncertainty, the momentum scale and resolution of leptons and jets, and the lepton reconstruction and selection efficiencies $[47,48,51,65]$. Smaller experimental uncertainties are also considered, which include uncertainties due to the trigger selection efficiency, the $b$-jet identification efficiency, the calculation of the $E_{\mathrm{T}}^{\text {miss }}$ soft-term, and the variation of the average number of interactions per bunch crossing (hereafter referred to as pile-up uncertainty). The pileup uncertainty covers the uncertainty on the ratio between the predicted and measured inelastic cross-section in the fiducial volume defined by $M_{X}>13 \mathrm{GeV}$ where $M_{X}$ is the mass of the hadronic system [66]. Overall, the total experimental uncertainty on $C_{Z Z}$ is $3.1 \%$, dominated by the jet and lepton components. The uncertainty in the combined $2015+2016$ integrated luminosity is $2.1 \%$. It is derived, following a methodology similar to that detailed in ref. [67], and using the LUCID-2 detector for the baseline luminosity measurements [68], from calibration of the luminosity scale using $\mathrm{x}-\mathrm{y}$ beam-separation scans.

The fractional uncertainties in $A_{Z Z}$ and $C_{Z Z}$ are summarised in table 5. In this analysis, the theoretical uncertainties are treated as uncorrelated between the $q q Z Z$ and $g g Z Z$ processes, while the experimental uncertainties are considered as fully correlated across the relevant processes and final-state channels.

\section{Integrated cross-section results}

Table 6 lists separately for the $e e$ and $\mu \mu$ channels the observed data yields and the expectations for the signal and background contributions after the final selection. Figure 5 shows for the combined $e e$ and $\mu \mu$ channels the observed and expected $E_{\mathrm{T}}^{\text {miss }}$ distributions, which are in good agreement.

The integrated fiducial and total cross-sections $\left(\sigma_{Z Z \rightarrow \ell \ell \nu \nu}^{\text {fid }}\right.$ and $\left.\sigma_{Z Z}^{\text {tot }}\right)$ are determined by binned maximum-likelihood fits to the $E_{\mathrm{T}}^{\text {miss }}$ distributions. As shown in figure 5 , the signal-to-background ratio increases as $E_{\mathrm{T}}^{\text {miss }}$ becomes larger. The use of $E_{\mathrm{T}}^{\text {miss }}$ improves the precision of the measured fiducial cross-section relatively by $5 \%$ compared with the case where no kinematic information is used. 


\begin{tabular}{|lcc|lcc|}
\hline \multicolumn{3}{|c|}{$A_{Z Z}$} & \multicolumn{3}{c|}{$C_{Z Z}$} \\
\hline & $q q Z Z$ & $g g Z Z$ & & $e e$ & $\mu \mu$ \\
Stat. & $1.0 \%$ & $1.1 \%$ & Stat. & $0.6 \%$ & $0.6 \%$ \\
PDF & $0.8 \%$ & $3.5 \%$ & Electron & $2.0 \%$ & - \\
Scale & $1.4 \%$ & $2.0 \%$ & Muon & - & $1.9 \%$ \\
UEPS & $0.1 \%$ & $2.0 \%$ & Set & $2.0 \%$ & $2.0 \%$ \\
Total & $1.9 \%$ & $4.6 \%$ & Total & $0.9 \%$ & $1.1 \%$ \\
\hline
\end{tabular}

Table 5. Fractional uncertainties for $A_{Z Z}$ and $C_{Z Z}$, with the contributions from the various sources, theoretical only for $A_{Z Z}$ and experimental only for $C_{Z Z}$. The uncertainties in $A_{Z Z}$ for the $q q Z Z$ and $g g Z Z$ sub-processes are given in different columns. The uncertainties in $C_{Z Z}$ for the ee and $\mu \mu$ channels of the inclusive $Z Z$ process are given in separate columns. The total uncertainties in $A_{Z Z}$ and $C_{Z Z}$ are given in the last rows, respectively. The "Soft" term includes the $E_{\mathrm{T}}^{\text {miss }}$ soft-term and the pile-up uncertainties.

\begin{tabular}{|c|c|c|}
\hline & $e e$ & $\mu \mu$ \\
\hline Data & 371 & 416 \\
\hline Signal & & \\
\hline$q q Z Z$ & $194 \pm 3 \pm 12$ & $202 \pm \quad 3 \pm 12$ \\
\hline$g g Z Z$ & $25.1 \pm 0.3 \pm 7.7$ & $26.4 \pm 0.3 \pm 8.1$ \\
\hline Backgrounds & & \\
\hline$W Z$ & $92.9 \pm 3.0 \pm 4.8$ & $100.7 \pm 3.2 \pm 5.2$ \\
\hline Non-resonant- $\ell \ell$ & $25.5 \pm 3.4 \pm 1.8$ & $31.5 \pm 4.2 \pm 2.2$ \\
\hline$Z+$ jets & $4.7 \pm 0.2 \pm 2.3$ & $5.9 \pm 0.3 \pm 2.8$ \\
\hline$Z Z \rightarrow 4 \ell$ & $3.8 \pm 0.2 \pm 0.3$ & $4.2 \pm 0.2 \pm 0.3$ \\
\hline Others & $0.87 \pm 0.03 \pm 0.17$ & $0.87 \pm 0.03 \pm 0.17$ \\
\hline Background expected & $128 \pm \quad 5 \pm$ & $143 \pm$ \\
\hline Total expected & $5 \pm 15$ & $6 \pm 16$ \\
\hline
\end{tabular}

Table 6. Observed data yields and expected signal and background contributions, shown separately for the $e e$ and $\mu \mu$ channels. The errors shown for the expected yields correspond to the statistical and systematic contributions in that order. The expected background and signal+background yields are shown in the last two rows, where the uncertainties are computed as the sum in quadrature of those from the individual processes.

The expected yield in each channel $i$ and in each $E_{\mathrm{T}}^{\text {miss }}$ bin $j$ is given by:

$$
N_{\exp }^{i j}=\sigma_{Z Z \rightarrow \ell \ell \nu \nu}^{\mathrm{fid}} \times \mathcal{L} \times C_{Z Z}^{i} \times f_{Z Z}^{i j}+N_{\mathrm{bkg}}^{i j}=\sigma_{Z Z}^{\mathrm{tot}} \times B \times \mathcal{L} \times A_{Z Z}^{i} \times C_{Z Z}^{i} \times f_{Z Z}^{i j}+N_{\mathrm{bkg}}^{i j},
$$

where $\mathcal{L}$ is the integrated luminosity, $N_{\text {bkg }}^{i j}$ the expected background yield, $B$ the branching fraction for the $Z Z \rightarrow \ell \ell \nu \nu$ decay $\left(\ell=e\right.$ or $\mu$ ), and $f_{Z Z}^{i j}$ is the fraction of signal events 


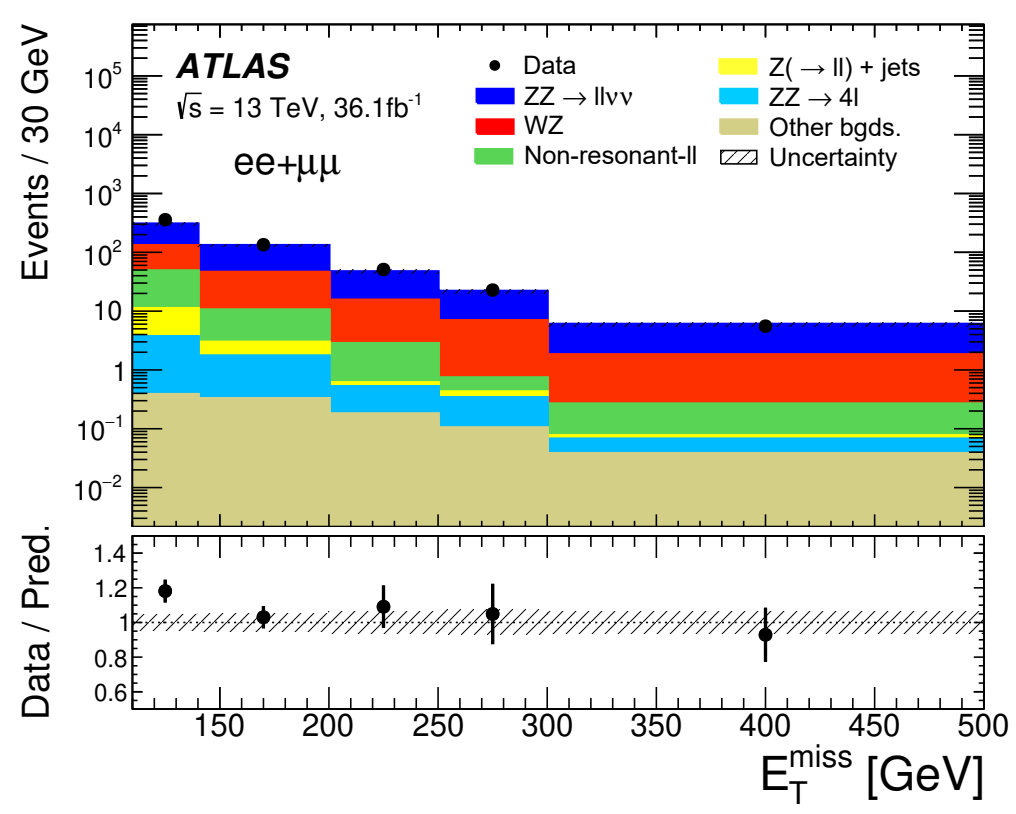

Figure 5. Observed and expected $E_{\mathrm{T}}^{\text {miss }}$ distributions after the final selection for the combined $e e$ $+\mu \mu$ channel before the fit procedure. The error bars on the data points correspond to the data statistical uncertainties, and the hashed band for the prediction includes both the statistical uncertainties of the simulation and the systematic uncertainties. The "other" background corresponds to the $V V V$ and $t \bar{t} V$ processes.

in bin $j$ with respect to the total distribution. The number of events follows a Poisson distribution in each bin, and the systematic uncertainties are treated as Gaussian nuisance parameters, $\theta_{k}$, in the fit. For each source of systematic uncertainty, $k$, a single nuisance parameter is used for all the processes and channels where this uncertainty matters. The statistical uncertainty due to the limited size of simulated samples is treated as uncorrelated among bins and channels. The binned likelihood function is built over all bins as follows:

$$
L(\sigma, \vec{\theta})=\prod_{i} \prod_{j} \operatorname{Pois}\left(N_{\mathrm{obs}}^{i j} \mid N_{\exp }^{i j}(\sigma, \vec{\theta})\right) \times \prod_{k} \operatorname{Gaus}\left(\theta_{k}\right)
$$

where $N_{\text {obs }}^{i j}$ is the observed data yield in each bin.

Table 7 summarises the main sources of uncertainty in the measured combined fiducial cross-section, where individual sources of a similar nature are grouped together. The statistical and total systematic uncertainties in the measurement are of similar sizes. Table 8 shows the measured fiducial cross-sections, separately for each channel and for their combination, together with the breakdown of their uncertainties. The $e e$ and $\mu \mu$ channel cross-sections are compatible within their respective statistical uncertainties. The measured combined fiducial cross-section has a total uncertainty of $7 \%$, which is significantly better than the previous measurement [7], and comparable in size to that obtained in the $Z Z \rightarrow 4 \ell$ channel $[8,12]$. Table 8 also shows the combined measured total cross-section, as well as the predictions for the cross-sections, as taken from table 4 . The combined 


\begin{tabular}{|l|cc|cccc|}
\hline & & & Lumi. & Electron & Muon & Jet \\
Total & Data stat. & Total syst. & $2.2 \%$ & $1.2 \%$ & $1.1 \%$ & $2.1 \%$ \\
\cline { 4 - 7 } $7.0 \%$ & $5.5 \%$ & $4.3 \%$ & $W Z$ & Non-resonant- $\ell \ell$ & $Z+$ jets & Sim. stat. \\
& & & $1.6 \%$ & $1.6 \%$ & $0.4 \%$ & $0.7 \%$ \\
\hline
\end{tabular}

Table 7. Relative contributions to the measured combined fiducial cross-section from the main sources of uncertainty after the fit procedure. The total uncertainty includes the data statistical and systematic components. For the systematic uncertainty, the individual sources of a similar nature are grouped together for simplicity. "Sim. stat." indicates the uncertainty source corresponding to the limited size of the simulation samples for the signal and background processes.

\begin{tabular}{|cccc|}
\hline & & Measured & Predicted \\
\hline & $e e$ & $12.2 \pm 1.0$ (stat) \pm 0.5 (syst) \pm 0.3 (lumi) & $11.2 \pm 0.6$ \\
$\sigma_{Z Z \rightarrow \ell \ell \nu \nu}^{\text {fid }}[\mathrm{fb}]$ & $\mu \mu$ & $13.3 \pm 1.0$ (stat) \pm 0.5 (syst) \pm 0.3 (lumi) & $11.2 \pm 0.6$ \\
& $e e+\mu \mu$ & $25.4 \pm 1.4$ (stat) \pm 0.9 (syst) \pm 0.5 (lumi) & $22.4 \pm 1.3$ \\
\hline$\sigma_{Z Z}^{\text {tot }}[\mathrm{pb}]$ & Total & $17.8 \pm 1.0$ (stat) \pm 0.7 (syst) \pm 0.4 (lumi) & $15.7 \pm 0.7$ \\
\hline
\end{tabular}

Table 8. Measured and predicted integrated cross-sections in the fiducial and total phase spaces, together with the breakdown of their uncertainties. The luminosity uncertainty is quoted separately from the other systematic uncertainties. The measurements are also shown separately for the ee and $\mu \mu$ channels in the case of the fiducial cross-section.

measurement is about $13 \%$ higher than the prediction, which is not significant given the size of the measurement and prediction uncertainties.

\section{Differential cross-section results}

Differential cross-sections are measured in the fiducial phase space by counting data events observed in each bin of the observables of interest, after subtracting the expected background contribution, and correcting for the detector effects with the unfolding procedure, chosen here to be the iterative Bayesian unfolding method of ref. [69]. The unfolding process takes into account fiducial corrections (correcting for events outside the fiducial phase space but passing the detector-level selections), bin-to-bin migrations due to detector resolution, and detector inefficiencies. An optimal number of two iterations is used for this analysis, as a balance between the size of the statistical uncertainty in the measurement and residual biases from the method. The residual bias is in almost all bins below 1\%, as estimated by comparing the results obtained using different prior distributions (constant, expected, observed) in the unfolding process.

The experimental uncertainties for the measurement results are evaluated by varying the response matrices for the unfolding according to the $\pm 1 \sigma$ effects of each uncertainty source, and by comparing the resulting unfolded results with the nominal one. The background uncertainties are considered at the stage of the background subtraction. The statistical uncertainty of the data is estimated by repeating the unfolding procedure with 2000 


\begin{tabular}{|l|cccccccc|}
\hline$p_{\mathrm{T}}^{\ell \ell}$ range $[\mathrm{GeV}]$ & $50-110$ & $110-130$ & $130-150$ & $150-170$ & $170-200$ & $200-250$ & $250-350$ & $350-1000$ \\
\hline Measured $\sigma(\mathrm{fb})$ & 9.3 & 6.6 & 3.6 & 2.1 & 2.5 & 2.0 & 1.1 & 0.4 \\
Total unc. & $17.7 \%$ & $13.6 \%$ & $15.2 \%$ & $18.6 \%$ & $18.6 \%$ & $17.6 \%$ & $24.9 \%$ & $40.5 \%$ \\
\hline Stat. unc. & $14.7 \%$ & $11.1 \%$ & $14.0 \%$ & $17.7 \%$ & $16.0 \%$ & $16.9 \%$ & $23.4 \%$ & $39.4 \%$ \\
Syst. unc. & $7.0 \%$ & $4.5 \%$ & $5.0 \%$ & $4.3 \%$ & $3.9 \%$ & $4.6 \%$ & $4.6 \%$ & $5.5 \%$ \\
Bkg. unc. & $6.9 \%$ & $6.4 \%$ & $3.2 \%$ & $3.7 \%$ & $8.6 \%$ & $2.1 \%$ & $7.1 \%$ & $7.6 \%$ \\
\hline Sim. stat. & $1.2 \%$ & $0.7 \%$ & $0.7 \%$ & $0.8 \%$ & $0.9 \%$ & $0.9 \%$ & $1.1 \%$ & $2.0 \%$ \\
Electron & $0.7 \%$ & $0.8 \%$ & $0.9 \%$ & $1.7 \%$ & $1.3 \%$ & $1.6 \%$ & $2.1 \%$ & $3.2 \%$ \\
Muon & $1.0 \%$ & $1.3 \%$ & $1.0 \%$ & $1.1 \%$ & $1.2 \%$ & $1.4 \%$ & $2.0 \%$ & $1.7 \%$ \\
Jet & $5.4 \%$ & $2.9 \%$ & $3.8 \%$ & $3.0 \%$ & $2.3 \%$ & $2.1 \%$ & $2.7 \%$ & $2.5 \%$ \\
Soft & $3.6 \%$ & $2.2 \%$ & $2.0 \%$ & $0.8 \%$ & $1.3 \%$ & $2.7 \%$ & $0.3 \%$ & $1.7 \%$ \\
Luminosity & $2.1 \%$ & $2.1 \%$ & $2.1 \%$ & $2.1 \%$ & $2.1 \%$ & $2.1 \%$ & $2.1 \%$ & $2.1 \%$ \\
\hline
\end{tabular}

Table 9. Measured cross-sections and breakdown of uncertainties (\%) for the unfolded $p_{\mathrm{T}}^{\ell \ell}$ distribution in the fiducial region. The top part of the table gives separately the three main contributions to the total uncertainty, arising respectively from data statistics (labelled Stat.), background subtraction (labelled Bkg.), and other systematic uncertainties (labelled Syst.). The bottom part of the table shows a more detailed breakdown of the third contribution (Syst.). The "Soft" term includes the $E_{\mathrm{T}}^{\mathrm{miss}}$ soft-term and the pile-up uncertainties.

sets of pseudo-data and then taking the root mean square of the deviations of the resulting spectra from the data spectrum. The response matrices are also subject to the statistical uncertainty of the simulated samples, which is estimated using a similar approach.

Figures 6 and 7 present the measured differential cross-sections for the eight observables of interest defined in section 1 . The binning for each variable is chosen to minimise the bin-to-bin migrations while preserving a sufficient number of events per bin. The $N_{\text {jets }}$ spectrum is measured only for hard jets as defined in section 4 , and the $p_{\mathrm{T}}^{\text {jet1 }}$ distribution is obtained in the fiducial phase space of events containing at least one hard jet. The predictions from Powheg+GG2Vv and Sherpa are also shown in figures 6 and 7 , and are found to be in agreement with the measurements within uncertainties. The electroweak production of $Z Z$ associated with two jets is not taken into account in the predictions due to its negligible contribution. The differential measurements are largely dominated by the statistical uncertainty on the data, but the systematic uncertainties contribute significantly in certain regions of phase space. As an example, the uncertainties from the various sources for the differential measurement of the $p_{\mathrm{T}}^{\ell \ell}$ distribution are listed in table 9 .

Comparing with the Run-1 results of ref. [7], this measurement is obtained from a larger dataset with highly improved accuracy and for a wider range of observables.

\section{Search for aTGCs}

The search for aTGCs is carried out using the unfolded $p_{\mathrm{T}}^{\ell \ell}$ distribution of figure 6 in the fiducial phase space. The contribution due to aTGCs is introduced using an effective vertex function approach [1]. It includes two coupling parameters that violate charge-parity (CP) 

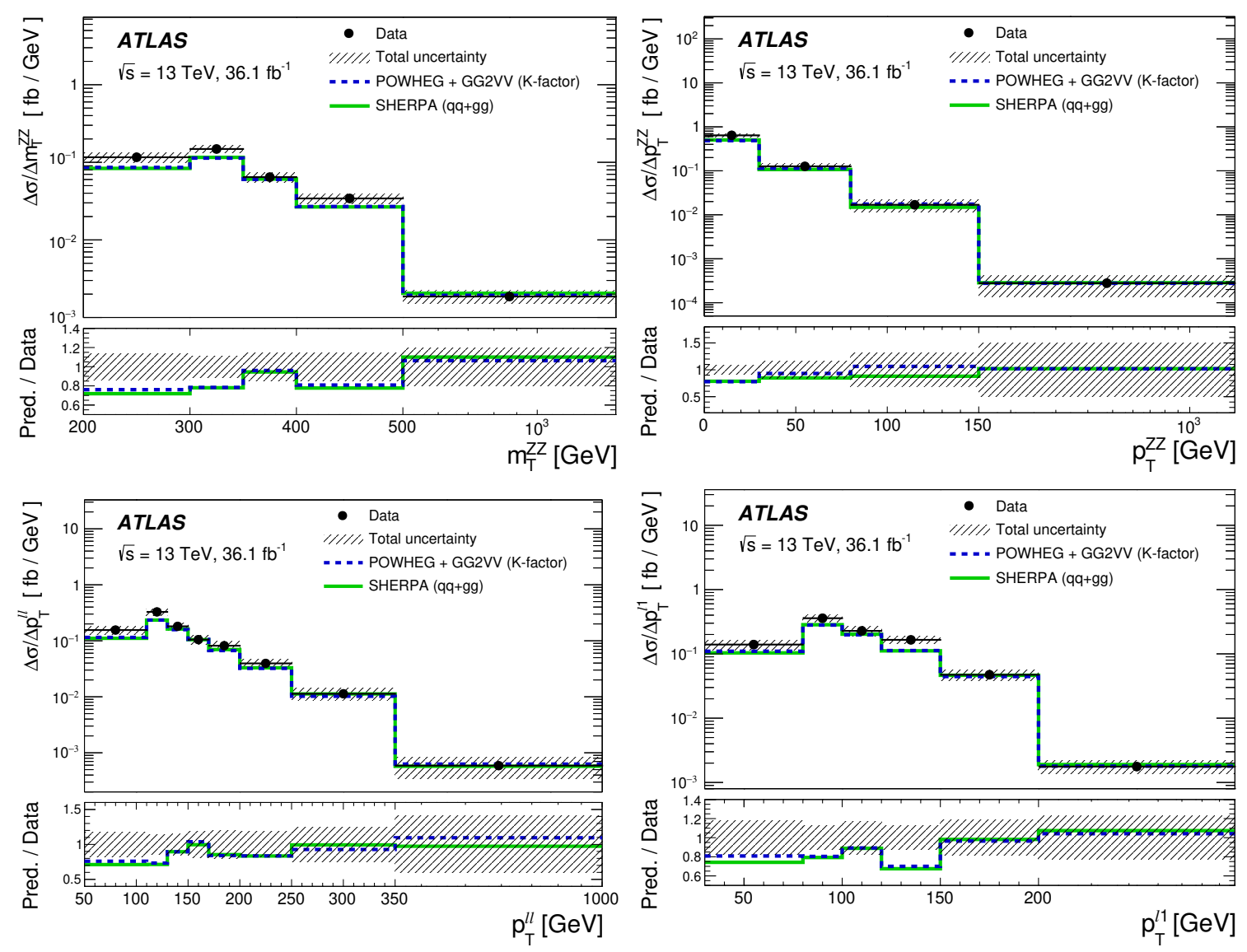

Figure 6. The measured differential cross-sections for $m_{\mathrm{T}}^{Z Z}$ (top left), $p_{\mathrm{T}}^{Z Z}$ (top right), $p_{\mathrm{T}}^{\ell \ell}$ (bottom left), and $p_{\mathrm{T}}^{\ell 1}$ (bottom right) in the fiducial region. The hashed bands in light grey give the total uncertainty. Also shown are the predictions as obtained from SHERPA (solid line) and PowHEG + GG2Vv (dashed line) with higher-order $K$-factors, as described in section 3 . The last bin in the four distributions is shown using a different $x$-axis scale for better visualisation.

symmetry, $f_{4}^{\gamma}$ and $f_{4}^{Z}$, as well as two CP-conserving ones, $f_{5}^{\gamma}$ and $f_{5}^{Z}$. The sensitivity range of this search is found to be within the unitarity bounds, so no form factors [1] are applied to the coupling parameters in this analysis. Furthermore, the coupling parameters are used to extract information about the dimension-eight operators of the effective field theory [70].

The relative change in the SM production cross-section in each $p_{\mathrm{T}}^{\ell \ell}$ bin is parameterised as a quadratic function depending on the coupling parameters. The parameterisation for the $q q Z Z$ process is derived from simulation as described in section 3, while the impact of the aTGCs is neglected for the $g g Z Z$ process. Since the sensitivity to possible aTGCs lies in the high- $p_{\mathrm{T}}^{\ell \ell}$ region, only the bins with $p_{\mathrm{T}}^{\ell \ell}>150 \mathrm{GeV}$ are considered in the search. Figure 8 compares the measured $p_{\mathrm{T}}^{\ell \ell}$ spectrum in this region of phase space to the SM prediction alone and to the SM prediction augmented with aTGCs corresponding to different values of the coupling parameters described above. The highest- $p_{\mathrm{T}}^{\ell \ell}$ bins are required to contain a minimum of ten observed events at the detector level, which arises from the fact that the limits on aTGCs derived from the unfolded distribution rely on a Gaussian 

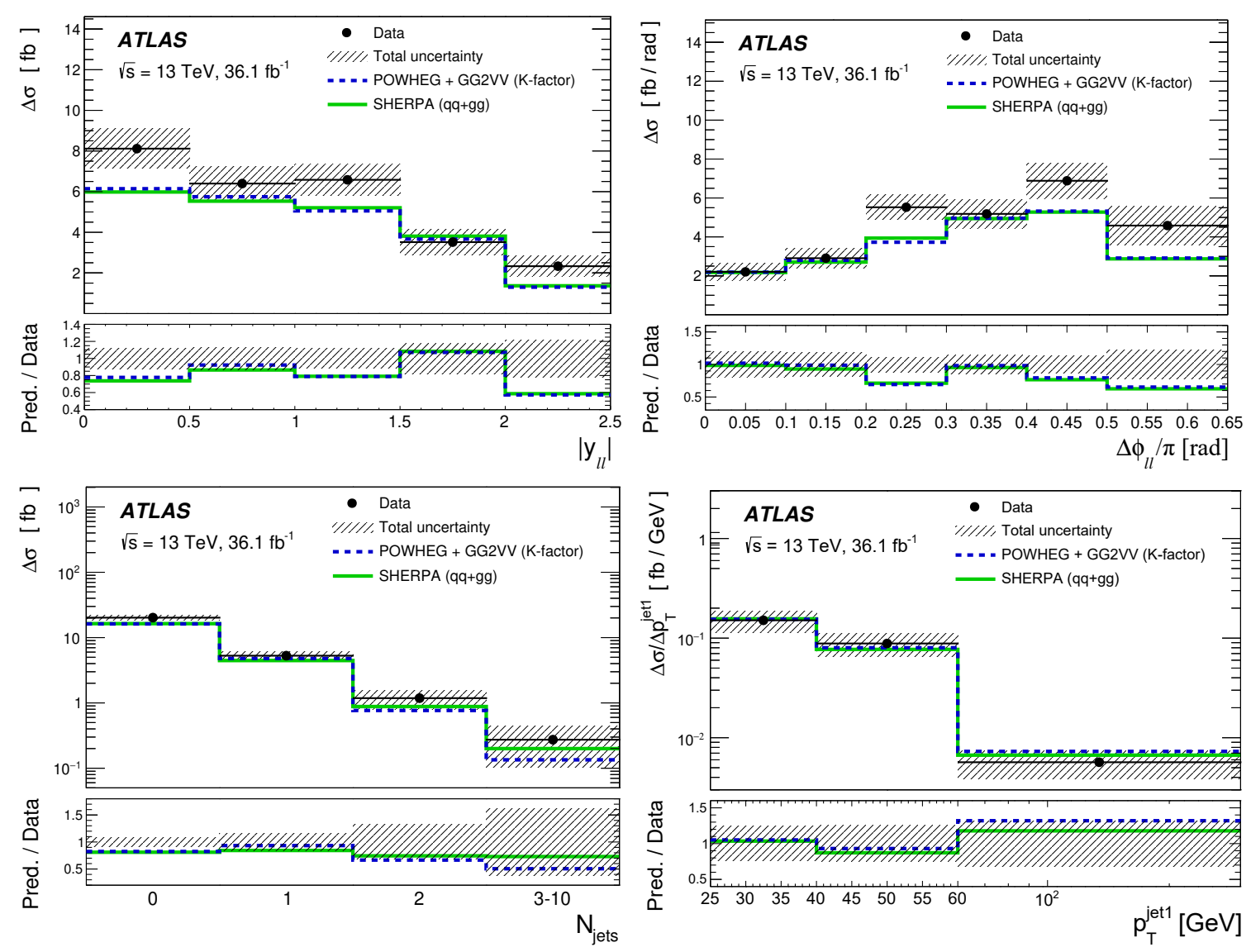

Figure 7. The measured differential cross-sections for $\left|y_{\ell \ell}\right|$ (top left), $\Delta \phi_{\ell \ell}$ (top right), $N_{\text {jets }}$ (bottom left), and $p_{\mathrm{T}}^{\text {jet1 }}$ (bottom right) in the fiducial region. The hashed bands in light grey give the total uncertainty. Also shown are the predictions as obtained from SHERPA (solid line) and PowheG +GG2VV (dashed line) with higher-order $K$-factors, as described in section 3 . The last bin in the distributions of $N_{\text {jets }}$ and $p_{\mathrm{T}}^{\text {jet1 }}$ is shown using a different $x$-axis scale for better visualisation.

approximation for the statistical fluctuations from the data. The total uncertainty in the measured cross-section in the last bin is about $40 \%$, while the corresponding SM prediction has an uncertainty of $15 \%$. The sensitivity to aTGCs is thus still limited by the statistical uncertainty of the data.

Since the observation is consistent with the SM prediction, 95\% confidence intervals (CIs) for the coupling parameters are derived. The test statistic is based on a profile loglikelihood ratio [71], and the likelihood function is described by a multivariate Gaussian distribution with the following form:

$$
L=\frac{1}{\sqrt{(2 \pi)^{5}|\mathcal{C}|}} \times \exp \left(-\frac{1}{2}(\vec{x}-\vec{\mu})^{T} \mathcal{C}^{-1}(\vec{x}-\vec{\mu})\right) \times \prod_{s} \operatorname{Gaus}\left(\theta_{s}\right),
$$

where $\vec{x}$ represents the measured $p_{\mathrm{T}}^{\ell \ell}$ spectrum, $\vec{\mu}$ the expected one, and $\mathcal{C}$ is the expected covariance matrix in the differential measurement, with the term $|\mathcal{C}|$ standing for the determinant of the covariance matrix. Both $\vec{x}$ and $\vec{\mu}$ have five elements, corresponding to the five 

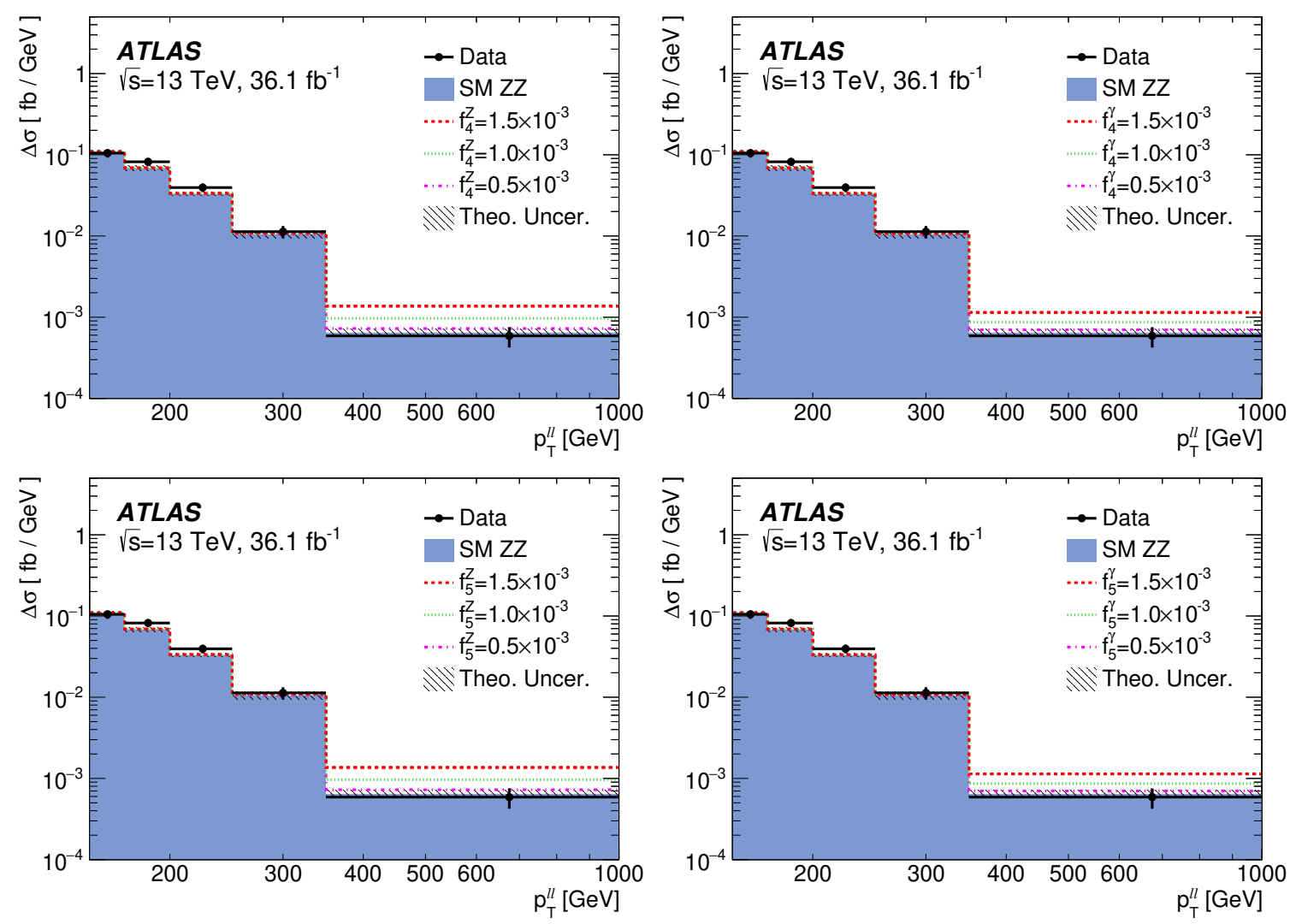

Figure 8. Unfolded measured distribution of $p_{\mathrm{T}}^{\ell \ell}$ for the bins with $p_{\mathrm{T}}^{\ell \ell}>150 \mathrm{GeV}$. The data are compared with the SM prediction from PowHEG+GG2Vv with higher-order $K$-factors to which are added possible aTGC contributions for different values of the strength of the coupling parameters defined in the text. The results are shown separately for $f_{4}^{Z}$ (top left), $f_{4}^{\gamma}$ (top right), $f_{5}^{Z}$ (bottom left), and $f_{5}^{\gamma}$ (bottom right). The uncertainty band represents the theoretical uncertainties in the SM predictions.

$p_{\mathrm{T}}^{\ell \ell}$ bins used in this search. The expectation $\vec{\mu}$ depends on the coupling parameters and a set of Gaussian nuisance parameters, $\theta_{s}$, which encapsulate the theoretical uncertainties in the prediction. The expected covariance matrix $\mathcal{C}$ is first derived by unfolding the predicted SM spectrum at detector level and then modified to account for the fact that the prediction $\vec{\mu}$, varied in the statistical test, differs from the SM prediction $\overrightarrow{\mu_{0}}$ in the fiducial region in the case of non-zero coupling parameters. The relative difference between $\vec{\mu}$ and $\overrightarrow{\mu_{0}}$ can be expressed using a vector $\vec{k}$, with $k^{i}=\mu^{i} / \mu_{0}^{i}$ for bin- $i$. Each matrix element $\mathcal{C}^{i j}$ is the sum of a statistical uncertainty component $\left(\mathcal{C}_{\text {stat }}^{i j}\right)$ and systematic uncertainty components arising from SM $Z Z$ process $\left(\mathcal{C}_{Z Z \text {-syst }}^{i j}\right)$ and background contribution $\left(\mathcal{C}_{\text {bkg-syst }}^{i j}\right)$; the dependence of $\mathcal{C}$ on $\vec{\mu}$ is implemented such that $\mathcal{C}_{\text {stat }}^{i j}$ and $\mathcal{C}_{Z Z \text {-syst }}^{i j}$ scale with $\sqrt{k^{i} \times k^{j}}$ and $k^{i} \times k^{j}$, respectively.

The $95 \%$ CIs are derived by scanning the parameter space and using a frequentist method with the $\mathrm{CL}_{\mathrm{s}}$ formalism [72]. In the first step, the one-dimensional CI for each aTGC parameter is derived with all other parameters set to zero, and the results are shown in table 10. These intervals are found to be more stringent than those obtained 


\begin{tabular}{|c|c|c|c|c|}
\hline & $f_{4}^{\gamma}$ & $f_{4}^{Z}$ & $f_{5}^{\gamma}$ & $f_{5}^{Z}$ \\
\hline Expected $\left[\times 10^{-3}\right]$ & {$[-1.3,1.3]$} & {$[-1.1,1.1]$} & {$[-1.3,1.3]$} & {$[-1.1,1.1]$} \\
Observed $\left[\times 10^{-3}\right]$ & {$[-1.2,1.2]$} & {$[-1.0,1.0]$} & {$[-1.2,1.2]$} & {$[-1.0,1.0]$} \\
\hline
\end{tabular}

Table 10. One-dimensional $95 \%$ confidence intervals for the aTGC parameters described in the text. The limits on each parameter are derived with the other parameters set to zero.

from the $Z Z \rightarrow 4 \ell$ channel based on the same ATLAS data sample [8]. In the second step, two-dimensional CIs for each pair of coupling parameters are derived with the other two parameters set to zero. Figure 9 presents the two-dimensional CI contours for the six possible pairs of aTGC coupling parameters. Finally, in the context of effective field theories, a one-dimensional 95\% CI can be placed on the CP-even dimension-eight operator $C_{\bar{B} W} / \Lambda^{4}[70]$. This is obtained by extrapolating the results of table 10 following the conversion formula in ref. [70]. A $95 \%$ CI of $[-4.0,4.0]$ in units of $\mathrm{TeV}^{-4}$ is set for $C_{\bar{B} W} / \Lambda^{4}$, which is improved by $30 \%$ with respect to the ATLAS results in the $Z Z \rightarrow 4 \ell$ channel [8].

\section{Conclusion}

This paper presents a measurement of $Z Z$ production using the $\ell \ell \nu \nu$ final state, based on $36.1 \mathrm{fb}^{-1}$ of data collected by the ATLAS detector at the LHC in $p p$ collisions at $\sqrt{s}=$ $13 \mathrm{TeV}$. The fiducial cross-section of the combined ee and $\mu \mu$ channels is measured to be $\sigma_{Z Z \rightarrow \ell \ell \nu \nu}^{\text {fid }}=25.4 \pm 1.4$ (stat) \pm 0.9 (syst) \pm 0.5 (lumi) fb, in agreement with the $\mathrm{SM}$ prediction of $22.4 \pm 1.3 \mathrm{fb}$. The integrated cross-sections in the total and fiducial phase spaces are measured with a total uncertainty of $7 \%$, which is significantly better than the previous measurement using the $8 \mathrm{TeV}$ data. The measured cross-sections are slightly larger than the SM predictions, but the difference is not significant considering the measurement and prediction uncertainties. Differential cross-sections are reported for eight different kinematic variables in the fiducial phase space, and no significant deviation from the expectations is found. The measured $p_{\mathrm{T}}^{\ell \ell}$ spectrum in the fiducial phase space is used to set limits on aTGCs, and the obtained $95 \%$ confidence intervals for the aTGC parameters are more stringent than those derived from the $4 \ell$ channel.

\section{Acknowledgments}

We thank CERN for the very successful operation of the LHC, as well as the support staff from our institutions without whom ATLAS could not be operated efficiently.

We acknowledge the support of ANPCyT, Argentina; YerPhI, Armenia; ARC, Australia; BMWFW and FWF, Austria; ANAS, Azerbaijan; SSTC, Belarus; CNPq and FAPESP, Brazil; NSERC, NRC and CFI, Canada; CERN; CONICYT, Chile; CAS, MOST and NSFC, China; COLCIENCIAS, Colombia; MSMT CR, MPO CR and VSC CR, Czech Republic; DNRF and DNSRC, Denmark; IN2P3-CNRS, CEA-DRF/IRFU, France; SRNSFG, Georgia; BMBF, HGF, and MPG, Germany; GSRT, Greece; RGC, Hong Kong 

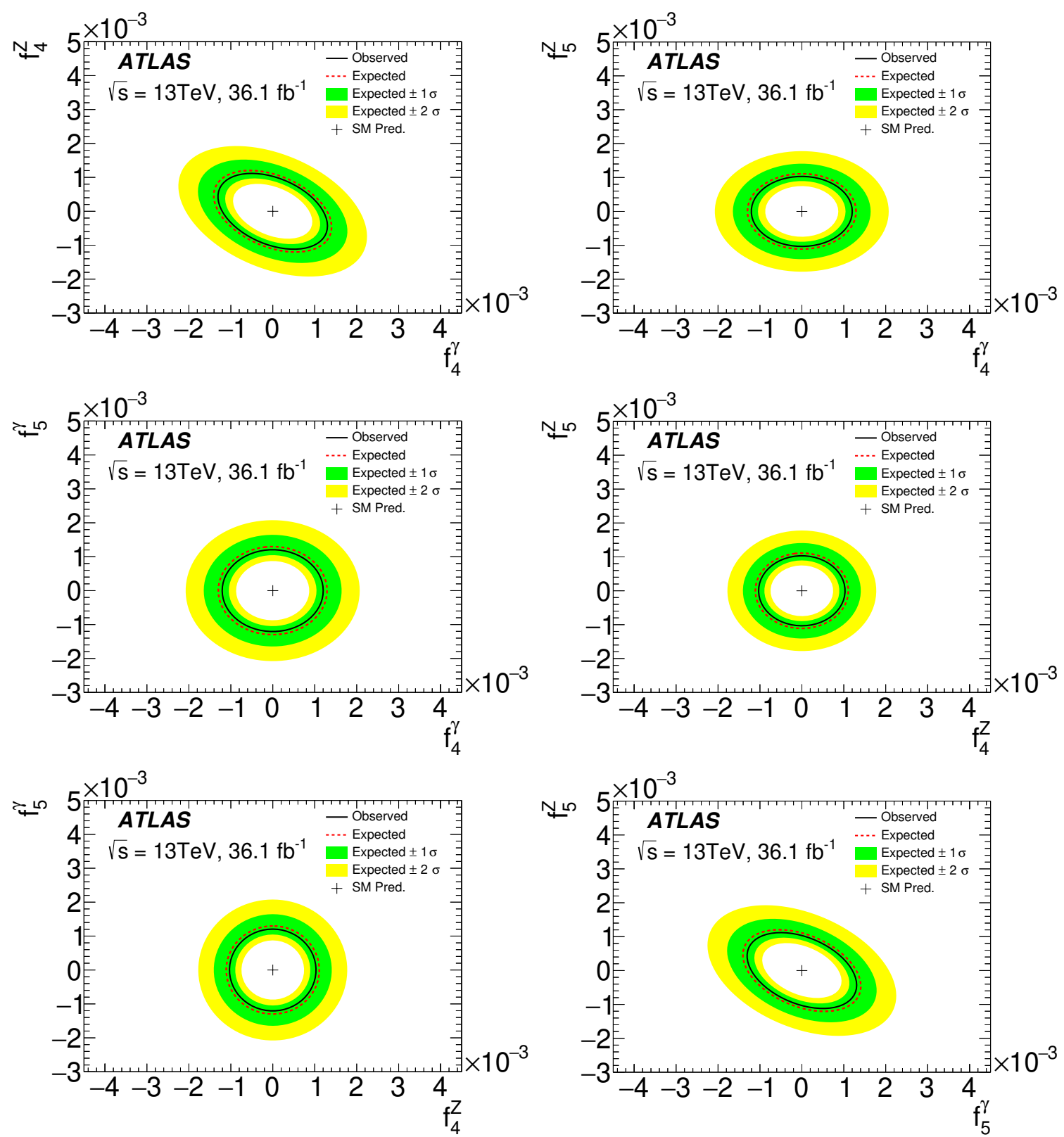

Figure 9. Expected and observed regions excluded at 95\% confidence level as a function of two aTGC parameters. Confidence intervals for each pair of coupling parameters are derived with the other two parameters set to zero.

SAR, China; ISF and Benoziyo Center, Israel; INFN, Italy; MEXT and JSPS, Japan; CNRST, Morocco; NWO, Netherlands; RCN, Norway; MNiSW and NCN, Poland; FCT, Portugal; MNE/IFA, Romania; MES of Russia and NRC KI, Russian Federation; JINR; MESTD, Serbia; MSSR, Slovakia; ARRS and MIZŠ, Slovenia; DST/NRF, South Africa; MINECO, Spain; SRC and Wallenberg Foundation, Sweden; SERI, SNSF and Cantons of Bern and Geneva, Switzerland; MOST, Taiwan; TAEK, Turkey; STFC, United Kingdom; DOE and NSF, United States of America. In addition, individual groups and members 
have received support from BCKDF, CANARIE, CRC and Compute Canada, Canada; COST, ERC, ERDF, Horizon 2020, and Marie Skłodowska-Curie Actions, European Union; Investissements d' Avenir Labex and Idex, ANR, France; DFG and AvH Foundation, Germany; Herakleitos, Thales and Aristeia programmes co-financed by EU-ESF and the Greek NSRF, Greece; BSF-NSF and GIF, Israel; CERCA Programme Generalitat de Catalunya, Spain; The Royal Society and Leverhulme Trust, United Kingdom.

The crucial computing support from all WLCG partners is acknowledged gratefully, in particular from CERN, the ATLAS Tier-1 facilities at TRIUMF (Canada), NDGF (Denmark, Norway, Sweden), CC-IN2P3 (France), KIT/GridKA (Germany), INFN-CNAF (Italy), NL-T1 (Netherlands), PIC (Spain), ASGC (Taiwan), RAL (U.K.) and BNL (U.S.A.), the Tier-2 facilities worldwide and large non-WLCG resource providers. Major contributors of computing resources are listed in ref. [73].

Open Access. This article is distributed under the terms of the Creative Commons Attribution License (CC-BY 4.0), which permits any use, distribution and reproduction in any medium, provided the original author(s) and source are credited.

\section{References}

[1] U. Baur and D.L. Rainwater, Probing neutral gauge boson selfinteractions in $Z Z$ production at hadron colliders, Phys. Rev. D 62 (2000) 113011 [hep-ph/0008063] [INSPIRE].

[2] D. Goncalves, T. Han and S. Mukhopadhyay, Off-Shell Higgs Probe of Naturalness, Phys. Rev. Lett. 120 (2018) 111801 [Erratum ibid. 121 (2018) 079902] [arXiv:1710.02149] [INSPIRE].

[3] ATLAS collaboration, Constraints on off-shell Higgs boson production and the Higgs boson total width in $Z Z \rightarrow 4 \ell$ and $Z Z \rightarrow 2 \ell 2 \nu$ final states with the ATLAS detector, Phys. Lett. B 786 (2018) 223 [arXiv:1808.01191] [INSPIRE].

[4] F. Cascioli et al., ZZ production at hadron colliders in NNLO QCD, Phys. Lett. B 735 (2014) 311 [arXiv:1405.2219] [INSPIRE].

[5] G.J. Gounaris, J. Layssac and F.M. Renard, New and standard physics contributions to anomalous $Z$ and gamma selfcouplings, Phys. Rev. D 62 (2000) 073013 [hep-ph/0003143] [INSPIRE].

[6] ATLAS collaboration, Measurement of $Z Z$ production in pp collisions at $\sqrt{s}=7 \mathrm{TeV}$ and limits on anomalous $Z Z Z$ and $Z Z \gamma$ couplings with the ATLAS detector, JHEP 03 (2013) 128 [arXiv:1211.6096] [INSPIRE].

[7] ATLAS collaboration, Measurement of the $Z Z$ production cross section in proton-proton collisions at $\sqrt{s}=8 \mathrm{TeV}$ using the $Z Z \rightarrow \ell^{-} \ell^{+} \ell^{\prime-} \ell^{\prime+}$ and $Z Z \rightarrow \ell^{-} \ell^{+} \nu \bar{\nu}$ channels with the ATLAS detector, JHEP 01 (2017) 099 [arXiv: 1610.07585] [INSPIRE].

[8] ATLAS collaboration, $Z Z \rightarrow \ell^{+} \ell^{-} \ell^{\prime+} \ell^{\prime-}$ cross-section measurements and search for anomalous triple gauge couplings in 13 TeV pp collisions with the ATLAS detector, Phys. Rev. D 97 (2018) 032005 [arXiv: 1709.07703] [INSPIRE].

[9] CMS collaboration, Measurement of the ZZ Production Cross Section and Search for

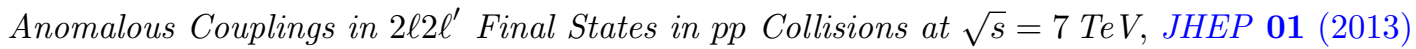
063 [arXiv:1211.4890] [INSPIRE]. 
[10] CMS collaboration, Measurements of the $Z Z$ production cross sections in the $2 \ell 2 \nu$ channel in proton-proton collisions at $\sqrt{s}=7$ and 8 TeV and combined constraints on triple gauge couplings, Eur. Phys. J. C 75 (2015) 511 [arXiv:1503.05467] [INSPIRE].

[11] CMS collaboration, Measurement of the $p p \rightarrow Z Z$ production cross section and constraints on anomalous triple gauge couplings in four-lepton final states at $\sqrt{s}=8$ TeV, Phys. Lett. B 740 (2015) 250 [arXiv:1406.0113] [INSPIRE].

[12] CMS collaboration, Measurements of the $p p \rightarrow Z Z$ production cross section and the $Z \rightarrow 4 \ell$ branching fraction and constraints on anomalous triple gauge couplings at $\sqrt{s}=13$ TeV, Eur. Phys. J. C 78 (2018) 165 [Erratum ibid. C 78 (2018) 515] [arXiv:1709.08601] [INSPIRE].

[13] M. Grazzini, S. Kallweit and D. Rathlev, ZZ production at the LHC: fiducial cross sections and distributions in NNLO QCD, Phys. Lett. B 750 (2015) 407 [arXiv:1507.06257] [INSPIRE].

[14] G. Heinrich, S. Jahn, S.P. Jones, M. Kerner and J. Pires, NNLO predictions for Z-boson pair production at the LHC, JHEP 03 (2018) 142 [arXiv: 1710.06294] [INSPIRE].

[15] B. Biedermann, A. Denner, S. Dittmaier, L. Hofer and B. Jäger, Electroweak corrections to $p p \rightarrow \mu^{+} \mu^{-} e^{+} e^{-}+X$ at the LHC: a Higgs background study, Phys. Rev. Lett. 116 (2016) 161803 [arXiv:1601.07787] [InSPIRE].

[16] S. Kallweit, J.M. Lindert, S. Pozzorini and M. Schönherr, NLO QCD + EW predictions for $2 \ell 2 \nu$ diboson signatures at the LHC, JHEP 11 (2017) 120 [arXiv:1705.00598] [INSPIRE].

[17] ATLAS collaboration, The ATLAS Experiment at the CERN Large Hadron Collider, 2008 JINST 3 S08003 [INSPIRE].

[18] ATLAS collaboration, Atlas Insertable B-Layer Technical Design Report, CERN-LHCC-2010-013 (2010) [ATLAS-TDR-19] [INSPIRE].

[19] ATLAS IBL collaboration, Production and Integration of the ATLAS Insertable B-Layer, 2018 JINST 13 T05008 [arXiv:1803.00844] [INSPIRE].

[20] ATLAS collaboration, Performance of the ATLAS Trigger System in 2015, Eur. Phys. J. C 77 (2017) 317 [arXiv:1611.09661] [INSPIRE].

[21] P. Nason, A New method for combining NLO QCD with shower Monte Carlo algorithms, JHEP 11 (2004) 040 [hep-ph/0409146] [INSPIRE].

[22] S. Frixione, P. Nason and C. Oleari, Matching NLO QCD computations with Parton Shower simulations: the POWHEG method, JHEP 11 (2007) 070 [arXiv:0709.2092] [INSPIRE].

[23] S. Alioli, P. Nason, C. Oleari and E. Re, A general framework for implementing NLO calculations in shower Monte Carlo programs: the POWHEG BOX, JHEP 06 (2010) 043 [arXiv: 1002.2581] [INSPIRE].

[24] P. Nason and G. Zanderighi, $W^{+} W^{-}, W Z$ and $Z Z$ production in the POWHEG-BOX-V2, Eur. Phys. J. C 74 (2014) 2702 [arXiv:1311.1365] [INSPIRE].

[25] T. Sjöstrand, S. Mrenna and P.Z. Skands, A Brief Introduction to PYTHIA 8.1, Comput. Phys. Commun. 178 (2008) 852 [arXiv:0710.3820] [INSPIRE].

[26] H.-L. Lai et al., New parton distributions for collider physics, Phys. Rev. D 82 (2010) 074024 [arXiv: 1007.2241] [INSPIRE].

[27] ATLAS collaboration, Measurement of the $Z / \gamma^{*}$ boson transverse momentum distribution in pp collisions at $\sqrt{s}=7$ TeV with the ATLAS detector, JHEP 09 (2014) 145 [arXiv: 1406.3660] [INSPIRE]. 
[28] J. Gao et al., CT10 next-to-next-to-leading order global analysis of QCD, Phys. Rev. D 89 (2014) 033009 [arXiv: 1302.6246] [INSPIRE].

[29] T. Gleisberg et al., Event generation with SHERPA 1.1, JHEP 02 (2009) 007 [arXiv:0811.4622] [INSPIRE].

[30] NNPDF collaboration, Parton distributions for the LHC Run II, JHEP 04 (2015) 040 [arXiv: 1410.8849] [INSPIRE].

[31] N. Kauer and G. Passarino, Inadequacy of zero-width approximation for a light Higgs boson signal, JHEP 08 (2012) 116 [arXiv:1206.4803] [INSPIRE].

[32] N. Kauer, Interference effects for $H \rightarrow W W / Z Z \rightarrow \ell \bar{\nu}_{\ell} \bar{\ell} \nu_{\ell}$ searches in gluon fusion at the LHC, JHEP 12 (2013) 082 [arXiv:1310.7011] [InSPIRE].

[33] ATLAS collaboration, ATLAS Run 1 PYTHIA8 tunes, ATL-PHYS-PUB-2014-021 (2014).

[34] F. Caola, K. Melnikov, R. Röntsch and L. Tancredi, QCD corrections to ZZ production in gluon fusion at the LHC, Phys. Rev. D 92 (2015) 094028 [arXiv: 1509.06734] [INSPIRE].

[35] F. Cascioli, S. Höche, F. Krauss, P. Maierhöfer, S. Pozzorini and F. Siegert, Precise Higgs-background predictions: merging NLO QCD and squared quark-loop corrections to four-lepton + 0,1 jet production, JHEP 01 (2014) 046 [arXiv:1309.0500] [INSPIRE].

[36] G. Bella, Weighting Di-Boson Monte Carlo Events in Hadron Colliders, arXiv:0803.3307 [INSPIRE].

[37] M. Bahr et al., HERWIG++ Physics and Manual, Eur. Phys. J. C 58 (2008) 639 [arXiv: 0803.0883] [INSPIRE].

[38] S. Frixione, P. Nason and G. Ridolfi, A Positive-weight next-to-leading-order Monte Carlo for heavy flavour hadroproduction, JHEP 09 (2007) 126 [arXiv:0707.3088] [INSPIRE].

[39] E. Re, Single-top Wt-channel production matched with parton showers using the POWHEG method, Eur. Phys. J. C 71 (2011) 1547 [arXiv: 1009.2450] [InSPIRE].

[40] T. Sjöstrand, S. Mrenna and P.Z. Skands, PYTHIA 6.4 Physics and Manual, JHEP 05 (2006) 026 [hep-ph/0603175] [INSPIRE].

[41] M. Czakon and A. Mitov, Top++: A Program for the Calculation of the Top-Pair Cross-Section at Hadron Colliders, Comput. Phys. Commun. 185 (2014) 2930 [arXiv: 1112.5675] [INSPIRE].

[42] N. Kidonakis, Two-loop soft anomalous dimensions for single top quark associated production with $a W^{-}$or $H^{-}$, Phys. Rev. D 82 (2010) 054018 [arXiv: 1005.4451] [InSPIRE].

[43] J. Alwall et al., The automated computation of tree-level and next-to-leading order differential cross sections and their matching to parton shower simulations, JHEP 07 (2014) 079 [arXiv: 1405.0301] [INSPIRE].

[44] ATLAS collaboration, The ATLAS Simulation Infrastructure, Eur. Phys. J. C 70 (2010) 823 [arXiv: 1005.4568] [INSPIRE].

[45] GEANT4 collaboration, GEANT4: A Simulation toolkit, Nucl. Instrum. Meth. A 506 (2003) 250 [INSPIRE].

[46] ATLAS collaboration, Further ATLAS tunes of PYTHIA6 and PYTHIA 8, ATL-PHYS-PUB-2011-014 (2011) [INSPIRE].

[47] ATLAS collaboration, Electron reconstruction and identification in the ATLAS experiment using the 2015 and 2016 LHC proton-proton collision data at $\sqrt{s}=13 \mathrm{TeV}$, Eur. Phys. J. C 79 (2019) 639 [arXiv:1902.04655] [INSPIRE]. 
[48] ATLAS collaboration, Muon reconstruction performance of the ATLAS detector in proton-proton collision data at $\sqrt{s}=13$ TeV, Eur. Phys. J. C 76 (2016) 292 [arXiv: 1603.05598] [INSPIRE].

[49] M. Cacciari, G.P. Salam and G. Soyez, The anti- $k_{t}$ jet clustering algorithm, JHEP 04 (2008) 063 [arXiv: 0802.1189] [INSPIRE].

[50] ATLAS collaboration, Topological cell clustering in the ATLAS calorimeters and its performance in LHC Run 1, Eur. Phys. J. C 77 (2017) 490 [arXiv: 1603. 02934] [inSPIRE].

[51] ATLAS collaboration, Jet energy scale measurements and their systematic uncertainties in proton-proton collisions at $\sqrt{s}=13 \mathrm{TeV}$ with the ATLAS detector, Phys. Rev. D 96 (2017) 072002 [arXiv: 1703.09665 ] [INSPIRE].

[52] M. Cacciari, G.P. Salam and G. Soyez, FastJet User Manual, Eur. Phys. J. C 72 (2012) 1896 [arXiv: 1111.6097] [INSPIRE].

[53] ATLAS collaboration, Performance of pile-up mitigation techniques for jets in pp collisions at $\sqrt{s}=8 \mathrm{TeV}$ using the ATLAS detector, Eur. Phys. J. C 76 (2016) 581 [arXiv: 1510.03823] [INSPIRE].

[54] ATLAS collaboration, Measurements of b-jet tagging efficiency with the ATLAS detector using $t \bar{t}$ events at $\sqrt{s}=13 \mathrm{TeV}$, JHEP 08 (2018) 089 [arXiv:1805.01845] [INSPIRE].

[55] ATLAS collaboration, Search for pair production of gluinos decaying via stop and sbottom in events with b-jets and large missing transverse momentum in pp collisions at $\sqrt{s}=13 \mathrm{TeV}$ with the ATLAS detector, Phys. Rev. D 94 (2016) 032003 [arXiv: 1605.09318] [InSPIRE].

[56] ATLAS collaboration, Performance of missing transverse momentum reconstruction with the ATLAS detector using proton-proton collisions at $\sqrt{s}=13$ TeV, Eur. Phys. J. C 78 (2018) 903 [arXiv: 1802.08168] [INSPIRE].

[57] Particle Data Group, The review of particle physics, Phys. Rev. D 98 (2018) 010001.

[58] ALEPH, DELPHI, L3, OPAL, SLD collaborations, LEP EleCtroweAK Working Group, SLD Electroweak Group and SLD Heavy Flavour Group, Precision electroweak measurements on the $Z$ resonance, Phys. Rept. 427 (2006) 257 [hep-ex/0509008] [INSPIRE].

[59] ATLAS collaboration, Measurement of $W^{ \pm} Z$ production cross sections and gauge boson polarisation in pp collisions at $\sqrt{s}=13 \mathrm{TeV}$ with the ATLAS detector, Eur. Phys. J. C 79 (2019) 535 [arXiv: 1902.05759] [INSPIRE].

[60] M. Grazzini, S. Kallweit, D. Rathlev and M. Wiesemann, $W^{ \pm} Z$ production at the LHC: fiducial cross sections and distributions in NNLO QCD, JHEP 05 (2017) 139 [arXiv: 1703.09065] [INSPIRE].

[61] ATLAS collaboration, Measurement of lepton differential distributions and the top quark mass in $t \bar{t}$ production in pp collisions at $\sqrt{s}=8 \mathrm{TeV}$ with the ATLAS detector, Eur. Phys. J. C 77 (2017) 804 [arXiv: 1709.09407] [INSPIRE].

[62] F. Maltoni, D. Pagani and I. Tsinikos, Associated production of a top-quark pair with vector bosons at NLO in QCD: impact on $t \bar{t} H$ searches at the LHC, JHEP 02 (2016) 113 [arXiv: 1507.05640] [INSPIRE].

[63] T. Binoth, G. Ossola, C.G. Papadopoulos and R. Pittau, NLO QCD corrections to tri-boson production, JHEP 06 (2008) 082 [arXiv:0804.0350] [INSPIRE].

[64] C.S. Li, H.T. Li, D.Y. Shao and J. Wang, Soft gluon resummation in the signal-background interference process of $g g\left(\rightarrow h^{*}\right) \rightarrow Z Z$, JHEP 08 (2015) 065 [arXiv: 1504.02388] [INSPIRE]. 
[65] ATLAS collaboration, Electron and photon energy calibration with the ATLAS detector using 2015-2016 LHC proton-proton collision data, 2019 JINST 14 P03017 [arXiv: 1812.03848] [INSPIRE].

[66] ATLAS collaboration, Measurement of the Inelastic Proton-Proton Cross Section at $\sqrt{s}=13$ TeV with the ATLAS Detector at the LHC, Phys. Rev. Lett. 117 (2016) 182002 [arXiv: 1606.02625] [INSPIRE].

[67] ATLAS collaboration, Luminosity determination in pp collisions at $\sqrt{s}=8 \mathrm{TeV}$ using the ATLAS detector at the LHC, Eur. Phys. J. C 76 (2016) 653 [arXiv:1608. 03953] [inSPIRE].

[68] G. Avoni et al., The new LUCID-2 detector for luminosity measurement and monitoring in ATLAS, 2018 JINST 13 P07017 [INSPIRE].

[69] G. D'Agostini, A Multidimensional unfolding method based on Bayes' theorem, Nucl. Instrum. Meth. A 362 (1995) 487 [INSPIRE].

[70] C. Degrande, A basis of dimension-eight operators for anomalous neutral triple gauge boson interactions, JHEP 02 (2014) 101 [arXiv:1308.6323] [INSPIRE].

[71] G. Cowan, K. Cranmer, E. Gross and O. Vitells, Asymptotic formulae for likelihood-based tests of new physics, Eur. Phys. J. C 71 (2011) 1554 [Erratum ibid. C 73 (2013) 2501] [arXiv: 1007.1727] [INSPIRE].

[72] A.L. Read, Presentation of search results: The CL technique, J. Phys. G 28 (2002) 2693 [INSPIRE].

[73] ATLAS collaboration, ATLAS Computing Acknowledgements, ATL-GEN-PUB-2016-002 (2016).

[74] M. Grazzini, S. Kallweit and M. Wiesemann, Fully differential NNLO computations with MATRIX, Eur. Phys. J. C 78 (2018) 537 [arXiv:1711.06631] [INSPIRE].

[75] S. Kallweit and M. Wiesemann, ZZ production at the $L H C$ : NNLO predictions for $2 \ell 2 \nu$ and $4 \ell$ signatures, Phys. Lett. B 786 (2018) 382 [arXiv:1806.05941] [INSPIRE].

[76] M. Grazzini, S. Kallweit, D. Rathlev and M. Wiesemann, $W^{ \pm} Z$ production at hadron colliders in NNLO QCD, Phys. Lett. B 761 (2016) 179 [arXiv:1604.08576] [INSPIRE]. 


\section{The ATLAS collaboration}

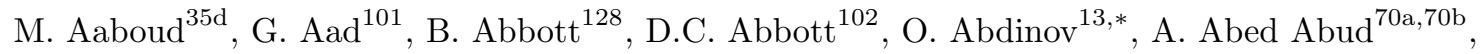
D.K. Abhayasinghe ${ }^{93}$, S.H. Abidi ${ }^{167}$, O.S. AbouZeid ${ }^{40}$, N.L. Abraham ${ }^{156}$, H. Abramowicz ${ }^{161}$, H. Abreu ${ }^{160}$, Y. Abulaiti ${ }^{6}$, B.S. Acharya ${ }^{66 a, 66 b, n}$, S. Adachi ${ }^{163}$, L. Adam ${ }^{99}$,

C. Adam Bourdarios ${ }^{132}$, L. Adamczyk ${ }^{83 a}$, L. Adamek ${ }^{167}$, J. Adelman ${ }^{121}$, M. Adersberger ${ }^{114}$, A. Adiguzel ${ }^{12 c, a h}$, S. Adorni ${ }^{54}$, T. Adye ${ }^{144}$, A.A. Affolder ${ }^{146}$, Y. Afik ${ }^{160}$, C. Agapopoulou ${ }^{132}$, M.N. Agaras ${ }^{38}$, A. Aggarwal ${ }^{119}$, C. Agheorghiesei ${ }^{27 c}$, J.A. Aguilar-Saavedra 140f,140a,ag, F. Ahmadov ${ }^{79}$, X. Ai ${ }^{15 a}$, G. Aielli ${ }^{73 a}, 73 b$, S. Akatsuka ${ }^{85}$, T.P.A. Åkesson ${ }^{96}$, E. Akilli ${ }^{54}$, A.V. Akimov ${ }^{110}$, K. Al Khoury ${ }^{132}$, G.L. Alberghi ${ }^{23 b, 23 a}{ }^{\text {, J. Albert }}{ }^{176}$, M.J. Alconada Verzini ${ }^{88}$, S. Alderweireldt ${ }^{119}$, M. Aleksa ${ }^{36}$, I.N. Aleksandrov ${ }^{79}$, C. Alexa ${ }^{27 b}$, D. Alexandre ${ }^{19}$, T. Alexopoulos ${ }^{10}$, A. Alfonsi ${ }^{120}$, M. Alhroob ${ }^{128}$, B. Ali ${ }^{142}$, G. Alimonti ${ }^{68 a}$, J. Alison ${ }^{37}$, S.P. Alkire ${ }^{148}$, C. Allaire ${ }^{132}$, B.M.M. Allbrooke ${ }^{156}$, B.W. Allen ${ }^{131}$, P.P. Allport ${ }^{21}$, A. Aloisio ${ }^{69 a, 69 b}$, A. Alonso ${ }^{40}$, F. Alonso ${ }^{88}$, C. Alpigiani ${ }^{148}$, A.A. Alshehri ${ }^{57}$, M.I. Alstaty ${ }^{101}$, M. Alvarez Estevez ${ }^{98}$, B. Alvarez Gonzalez ${ }^{36}$, D. Álvarez Piqueras ${ }^{174}$, M.G. Alviggi ${ }^{69 a, 69 b}$, Y. Amaral Coutinho ${ }^{80 b}$, A. Ambler ${ }^{103}$, L. Ambroz ${ }^{135}$, C. Amelung ${ }^{26}$, D. Amidei ${ }^{105}$, S.P. Amor Dos Santos ${ }^{140 a, 140 c}$, S. Amoroso ${ }^{46}$, C.S. Amrouche ${ }^{54}$, F. An ${ }^{78}$, C. Anastopoulos ${ }^{149}$, N. Andari ${ }^{145}$, T. Andeen ${ }^{11}$, C.F. Anders ${ }^{61 b}$, J.K. Anders ${ }^{20}$, A. Andreazza ${ }^{68 a, 68 b}$, V. Andrei ${ }^{61 a}$, C.R. Anelli ${ }^{176}$, S. Angelidakis ${ }^{38}$, I. Angelozzi ${ }^{120}$, A. Angerami ${ }^{39}$, A.V. Anisenkov ${ }^{122 b, 122 a}$, A. Annovi ${ }^{71 a}$, C. Antel ${ }^{61 a}$, M.T. Anthony ${ }^{149}$, M. Antonelli ${ }^{51}$, D.J.A. Antrim ${ }^{171}$, F. Anulli ${ }^{72 a}$, M. Aoki ${ }^{81}$, J.A. Aparisi Pozo ${ }^{174}$, L. Aperio Bella ${ }^{36}$, G. Arabidze ${ }^{106}$, J.P. Araque ${ }^{140 a}$, V. Araujo Ferraz ${ }^{80 b}$, R. Araujo Pereira ${ }^{80 b}$, A.T.H. Arce ${ }^{49}$, F.A. Arduh ${ }^{88}$, J-F. Arguin ${ }^{109}$, S. Argyropoulos ${ }^{77}$, J.-H. Arling ${ }^{46}$, A.J. Armbruster ${ }^{36}$, L.J. Armitage ${ }^{92}$, A. Armstrong ${ }^{171}$, O. Arnaez ${ }^{167}$, H. Arnold ${ }^{120}$, A. Artamonov ${ }^{111, *}$, G. Artoni ${ }^{135}$, S. Artz ${ }^{99}$, S. Asai ${ }^{163}$, N. Asbah ${ }^{59}$, E.M. Asimakopoulou ${ }^{172}$, L. Asquith ${ }^{156}$, K. Assamagan ${ }^{29}$, R. Astalos ${ }^{28 a}$, R.J. Atkin ${ }^{33 a}$, M. Atkinson ${ }^{173}$, N.B. Atlay ${ }^{151}$, H. Atmani ${ }^{132}$, K. Augsten ${ }^{142}$, G. Avolio ${ }^{36}$, R. Avramidou ${ }^{60 a}$, M.K. Ayoub ${ }^{15 a}$, A.M. Azoulay ${ }^{168 b}$, G. Azuelos ${ }^{109, a v}$, A.E. Baas ${ }^{61 a}$, M.J. Baca ${ }^{21}$, H. Bachacou ${ }^{145}$, K. Bachas ${ }^{67 a, 67 b}$, M. Backes ${ }^{135}$, F. Backman ${ }^{45 a, 45 b}$, P. Bagnaia ${ }^{72 a, 72 b}$, M. Bahmani ${ }^{84}$, H. Bahrasemani ${ }^{152}$, A.J. Bailey ${ }^{174}$, V.R. Bailey ${ }^{173}$, J.T. Baines ${ }^{144}$, M. Bajic ${ }^{40}$, C. Bakalis ${ }^{10}$, O.K. Baker ${ }^{183}$, P.J. Bakker ${ }^{120}$, D. Bakshi Gupta ${ }^{8}$, S. Balaji ${ }^{157}$, E.M. Baldin ${ }^{122 b, 122 a}$, P. Balek ${ }^{180}$, F. Balli ${ }^{145}$, W.K. Balunas ${ }^{135}$, J. Balz ${ }^{99}$, E. Banas ${ }^{84}$, A. Bandyopadhyay ${ }^{24}$, Sw. Banerjee ${ }^{181, i}$, A.A.E. Bannoura ${ }^{182}$, L. Barak ${ }^{161}$, W.M. Barbe ${ }^{38}$, E.L. Barberio ${ }^{104}$, D. Barberis ${ }^{55 b, 55 a}$, M. Barbero ${ }^{101}$, T. Barillari ${ }^{115}$, M-S. Barisits ${ }^{36}$, J. Barkeloo ${ }^{131}$, T. Barklow ${ }^{153}$, R. Barnea ${ }^{160}$, S.L. Barnes $^{60 c}$, B.M. Barnett ${ }^{144}$, R.M. Barnett ${ }^{18}$, Z. Barnovska-Blenessy ${ }^{60 a}$, A. Baroncelli ${ }^{60 a}$, G. Barone ${ }^{29}$, A.J. Barr ${ }^{135}$, L. Barranco Navarro ${ }^{174}$, F. Barreiro ${ }^{98}$,

J. Barreiro Guimarães da Costa ${ }^{15 a}$, R. Bartoldus ${ }^{153}$, G. Bartolini ${ }^{101}$, A.E. Barton ${ }^{89}$, P. Bartos ${ }^{28 a}$, A. Basalaev ${ }^{46}$, A. Bassalat ${ }^{132, \text { ap }}$, R.L. Bates ${ }^{57}$, S.J. Batista ${ }^{167}$, S. Batlamous ${ }^{35 e}$, J.R. Batley ${ }^{32}$, B. Batool ${ }^{151}$, M. Battaglia ${ }^{146}$, M. Bauce ${ }^{72 a, 72 b}$, F. Bauer ${ }^{145}$, K.T. Bauer ${ }^{171}$, H.S. Bawa ${ }^{31,1}$, J.B. Beacham ${ }^{49}$, T. Beau ${ }^{136}$, P.H. Beauchemin ${ }^{170}$, P. Bechtle ${ }^{24}$, H.C. Beck ${ }^{53}$, H.P. Beck ${ }^{20, q}$, K. Becker ${ }^{52}$, M. Becker ${ }^{99}$, C. Becot ${ }^{46}$, A. Beddall ${ }^{12 \mathrm{~d}}$, A.J. Beddall ${ }^{12 \mathrm{a}}$, V.A. Bednyakov ${ }^{79}$,

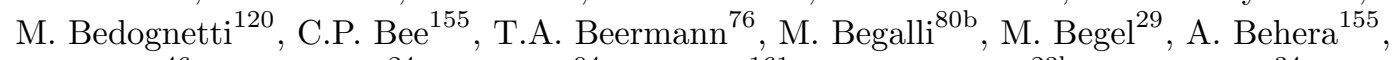
J.K. Behr ${ }^{46}$, F. Beisiegel ${ }^{24}$, A.S. Bell ${ }^{94}$, G. Bella ${ }^{161}$, L. Bellagamba ${ }^{23 b}$, A. Bellerive ${ }^{34}$, P. Bellos ${ }^{9}$, K. Beloborodov ${ }^{122 b, 122 a}$, K. Belotskiy ${ }^{112}$, N.L. Belyaev ${ }^{112}$, O. Benary ${ }^{161, *}$, D. Benchekroun ${ }^{35 a}$, N. Benekos ${ }^{10}$, Y. Benhammou ${ }^{161}$, D.P. Benjamin ${ }^{6}$, M. Benoit ${ }^{54}$, J.R. Bensinger ${ }^{26}$,

S. Bentvelsen ${ }^{120}$, L. Beresford ${ }^{135}$, M. Beretta ${ }^{51}$, D. Berge ${ }^{46}$, E. Bergeaas Kuutmann ${ }^{172}$, N. Berger ${ }^{5}$, B. Bergmann ${ }^{142}$, L.J. Bergsten ${ }^{26}$, J. Beringer ${ }^{18}$, S. Berlendis ${ }^{7}$, N.R. Bernard ${ }^{102}$, G. Bernardi ${ }^{136}$, C. Bernius ${ }^{153}$, F.U. Bernlochner ${ }^{24}$, T. Berry ${ }^{93}$, P. Berta ${ }^{99}$, C. Bertella ${ }^{15 a}$, G. Bertoli $^{45 a, 45 b}$, I.A. Bertram ${ }^{89}$, G.J. Besjes ${ }^{40}$, O. Bessidskaia Bylund ${ }^{182}$, N. Besson ${ }^{145}$, 
A. Bethani ${ }^{100}$, S. Bethke ${ }^{115}$, A. Betti ${ }^{24}$, A.J. Bevan ${ }^{92}$, J. Beyer ${ }^{115}$, R. Bi ${ }^{139}$, R.M. Bianchi ${ }^{139}$, O. Biebel ${ }^{114}$, D. Biedermann ${ }^{19}$, R. Bielski ${ }^{36}$, K. Bierwagen ${ }^{99}$, N.V. Biesuz ${ }^{71 a, 71 b}$, M. Biglietti $^{74 a}$, T.R.V. Billoud ${ }^{109}$, M. Bindi ${ }^{53}$, A. Bingul ${ }^{12 \mathrm{~d}}$, C. Bini ${ }^{72 a, 72 b}$, S. Biondi ${ }^{23 b, 23 a}$, M. Birman ${ }^{180}$, T. Bisanz ${ }^{53}$, J.P. Biswal ${ }^{161}$, A. Bitadze ${ }^{100}$, C. Bittrich ${ }^{48}$, D.M. Bjergaard ${ }^{49}$, J.E. Black ${ }^{153}$, K.M. Black ${ }^{25}$, T. Blazek ${ }^{28 a}$, I. Bloch ${ }^{46}$, C. Blocker ${ }^{26}$, A. Blue ${ }^{57}$, U. Blumenschein ${ }^{92}$, G.J. Bobbink ${ }^{120}$, V.S. Bobrovnikov ${ }^{122 b, 122 a}$, S.S. Bocchetta ${ }^{96}$, A. Bocci ${ }^{49}$, D. Boerner ${ }^{46}$, D. Bogavac ${ }^{114}$, A.G. Bogdanchikov ${ }^{122 b, 122 a}$, C. Bohm ${ }^{45 a}$, V. Boisvert ${ }^{93}$, P. Bokan ${ }^{53,172}$, T. Bold ${ }^{83 a}$, A.S. Boldyrev ${ }^{113}$, A.E. Bolz ${ }^{61 b}$, M. Bomben ${ }^{136}$, M. Bona ${ }^{92}$, J.S. Bonilla ${ }^{131}$, M. Boonekamp ${ }^{145}$, H.M. Borecka-Bielska ${ }^{90}$, A. Borisov ${ }^{123}$, G. Borissov ${ }^{89}$, J. Bortfeldt ${ }^{36}$, D. Bortoletto ${ }^{135}$, V. Bortolotto ${ }^{73 a, 73 b}$, D. Boscherini ${ }^{23 b}$, M. Bosman ${ }^{14}$, J.D. Bossio Sola ${ }^{30}$, K. Bouaouda ${ }^{35 a}$, J. Boudreau ${ }^{139}$, E.V. Bouhova-Thacker ${ }^{89}$, D. Boumediene ${ }^{38}$, S.K. Boutle ${ }^{57}$, A. Boveia ${ }^{126}$, J. Boyd ${ }^{36}$, D. Boye ${ }^{33 b}$, I.R. Boyko ${ }^{79}$, A.J. Bozson ${ }^{93}$, J. Bracinik ${ }^{21}$, N. Brahimi ${ }^{101}$, G. Brandt ${ }^{182}$, O. Brandt ${ }^{61 a}$, F. Braren ${ }^{46}$, U. Bratzler ${ }^{164}$, B. Brau ${ }^{102}$, J.E. Brau ${ }^{131}$, W.D. Breaden Madden ${ }^{57}$, K. Brendlinger ${ }^{46}$, L. Brenner ${ }^{46}$, R. Brenner ${ }^{172}$, S. Bressler ${ }^{180}$, B. Brickwedde ${ }^{99}$, D.L. Briglin ${ }^{21}$, D. Britton ${ }^{57}$, D. Britzger ${ }^{115}$, I. Brock ${ }^{24}$, R. Brock ${ }^{106}$, G. Brooijmans ${ }^{39}$, T. Brooks ${ }^{93}$, W.K. Brooks ${ }^{147 \mathrm{~b}}$, E. Brost ${ }^{121}$, J.H Broughton ${ }^{21}$, P.A. Bruckman de Renstrom ${ }^{84}$, D. Bruncko ${ }^{28 b}$, A. Bruni ${ }^{23 b}$, G. Bruni ${ }^{23 b}$, L.S. Bruni ${ }^{120}$, S. Bruno ${ }^{73 a, 73 b}$, B.H. Brunt ${ }^{32}$, M. Bruschi ${ }^{23 b}$, N. Bruscino ${ }^{139}$, P. Bryant ${ }^{37}$, L. Bryngemark ${ }^{96}$, T. Buanes ${ }^{17}$, Q. Buat ${ }^{36}$, P. Buchholz ${ }^{151}$, A.G. Buckley ${ }^{57}$, I.A. Budagov ${ }^{79}$, M.K. Bugge ${ }^{134}$, F. Bührer ${ }^{52}$, O. Bulekov ${ }^{112}$, T.J. Burch ${ }^{121}$, S. Burdin ${ }^{90}$, C.D. Burgard ${ }^{120}$, A.M. Burger ${ }^{129}$, B. Burghgrave ${ }^{8}$, K. Burka ${ }^{84}$, J.T.P. Burr ${ }^{46}$, V. Büscher ${ }^{99}$, E. Buschmann ${ }^{53}$, P.J. Bussey ${ }^{57}$, J.M. Butler ${ }^{25}$, C.M. Buttar ${ }^{57}$, J.M. Butterworth ${ }^{94}$, P. Butti ${ }^{36}$, W. Buttinger ${ }^{36}$, A. Buzatu ${ }^{158}$, A.R. Buzykaev ${ }^{122 b, 122 a}$, G. Cabras ${ }^{23 b, 23 a}$, S. Cabrera Urbán ${ }^{174}$, D. Caforio ${ }^{142}, \mathrm{H}$ Cai $^{173}$, V.M.M. Cairo ${ }^{153}$, O. Cakir ${ }^{4 a}$, N. Calace ${ }^{36}$, P. Calafiura ${ }^{18}$, A. Calandri ${ }^{101}$, G. Calderini ${ }^{136}$, P. Calfayan ${ }^{65}$, G. Callea ${ }^{57}$, L.P. Caloba ${ }^{80 b}$, S. Calvente Lopez ${ }^{98}$, D. Calvet ${ }^{38}$, S. Calvet ${ }^{38}$, T.P. Calvet ${ }^{155}$, M. Calvetti ${ }^{71 a, 71 b}$, R. Camacho Toro ${ }^{136}$, S. Camarda $^{36}$, D. Camarero Munoz ${ }^{98}$, P. Camarri ${ }^{73 a, 73 b}$, D. Cameron ${ }^{134}$, R. Caminal Armadans ${ }^{102}$, C. Camincher ${ }^{36}$, S. Campana ${ }^{36}$, M. Campanelli ${ }^{94}$, A. Camplani ${ }^{40}$, A. Campoverde ${ }^{151}$, V. Canale ${ }^{69 a, 69 b}$, A. Canesse ${ }^{103}$, M. Cano Bret ${ }^{60 c}$, J. Cantero ${ }^{129}$, T. Cao ${ }^{161}$, Y. Cao ${ }^{173}$, M.D.M. Capeans Garrido ${ }^{36}$, M. Capua ${ }^{41 \mathrm{~b}, 41 \mathrm{a}}$, R. Cardarelli ${ }^{73 a}$, F.C. Cardillo ${ }^{149}$, I. Carli ${ }^{143}$, T. Carli ${ }^{36}$, G. Carlino ${ }^{69 a}$, B.T. Carlson ${ }^{139}$, L. Carminati ${ }^{68 a, 68 b}$, R.M.D. Carney ${ }^{45 a, 45 b}$, S. Caron ${ }^{119}$, E. Carquin ${ }^{147 b}$, S. Carrá ${ }^{68 a, 68 b}$, J.W.S. Carter ${ }^{167}$, M.P. Casado ${ }^{14, e}$, A.F. Casha ${ }^{167}$, D.W. Casper ${ }^{171}$, R. Castelijn ${ }^{120}$, F.L. Castillo ${ }^{174}$, V. Castillo Gimenez ${ }^{174}$, N.F. Castro ${ }^{140 a, 140 e}$, A. Catinaccio ${ }^{36}$, J.R. Catmore ${ }^{134}$, A. Cattai $^{36}$, J. Caudron ${ }^{24}$, V. Cavaliere ${ }^{29}$, E. Cavallaro ${ }^{14}$, D. Cavalli ${ }^{68 a}$, M. Cavalli-Sforza ${ }^{14}$, V. Cavasinni ${ }^{71 a, 71 b}$, E. Celebi ${ }^{12 b}$, F. Ceradini ${ }^{74 a, 74 b}$, L. Cerda Alberich $^{174}$, A.S. Cerqueira ${ }^{80 a}$, A. Cerri ${ }^{156}$, L. Cerrito ${ }^{73 a, 73 b}$, F. Cerutti $^{18}$, A. Cervelli ${ }^{23 b, 23 a}$, S.A. Cetin ${ }^{12 b}$, A. Chafaq ${ }^{35 a}$, D. Chakraborty ${ }^{121}$, S.K. Chan ${ }^{59}$, W.S. Chan ${ }^{120}$, W.Y. Chan ${ }^{90}$, J.D. Chapman ${ }^{32}$, B. Chargeishvili ${ }^{159 b}$, D.G. Charlton ${ }^{21}$, C.C. $\mathrm{Chau}^{34}$, C.A. Chavez Barajas ${ }^{156}$, S. Che ${ }^{126}$, A. Chegwidden ${ }^{106}$, S. Chekanov ${ }^{6}$, S.V. Chekulaev ${ }^{168 a}$, G.A. Chelkov ${ }^{79, a u}$, M.A. Chelstowska ${ }^{36}$, B. Chen ${ }^{78}$, C. Chen ${ }^{60 a}$, C.H. Chen ${ }^{78}$, H. Chen ${ }^{29}$, J. Chen ${ }^{60 a}$, J. Chen ${ }^{39}$, S. Chen ${ }^{137}$, S.J. Chen ${ }^{15 c}$, X. Chen ${ }^{15 b \text {,at }}$, Y. Chen ${ }^{82}$, Y-H. Chen ${ }^{46}$, H.C. Cheng ${ }^{63 a}$, H.J. Cheng ${ }^{15 a, 15 d}$, A. Cheplakov ${ }^{79}$, E. Cheremushkina ${ }^{123}$, R. Cherkaoui El Moursli ${ }^{35 e}$, E. Cheu ${ }^{7}$, K. Cheung ${ }^{64}$, T.J.A. Chevalérias ${ }^{145}$, L. Chevalier ${ }^{145}$, V. Chiarella ${ }^{51}$, G. Chiarelli ${ }^{71 a}$, G. Chiodini ${ }^{67 a}$, A.S. Chisholm ${ }^{36,21}$, A. Chitan ${ }^{27 b}$, I. Chiu ${ }^{163}$, Y.H. Chiu ${ }^{176}$, M.V. Chizhov ${ }^{79}$, K. Choi ${ }^{65}$, A.R. Chomont ${ }^{132}$, S. Chouridou ${ }^{162}$, Y.S. Chow ${ }^{120}$, M.C. Chu ${ }^{63 a}$, J. Chudoba ${ }^{141}$, A.J. Chuinard ${ }^{103}$, J.J. Chwastowski ${ }^{84}$, L. Chytka ${ }^{130}$,

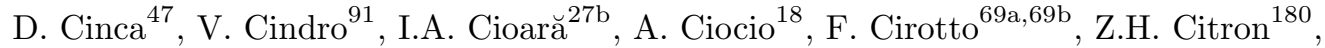
M. Citterio ${ }^{68 a}$, B.M. Ciungu ${ }^{167}$, A. Clark ${ }^{54}$, M.R. Clark $^{39}$, P.J. Clark ${ }^{50}$, C. Clement ${ }^{45 a, 45 b}$,

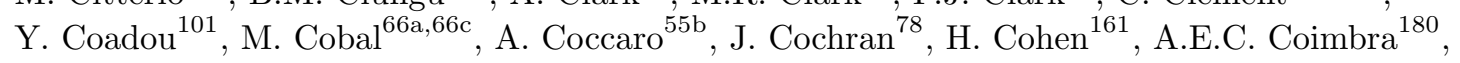


L. Colasurdo ${ }^{119}$, B. Cole $^{39}$, A.P. Colijn ${ }^{120}$, J. Collot ${ }^{58}$, P. Conde Muiño ${ }^{140 a, f}$, E. Coniavitis ${ }^{52}$, S.H. Connell ${ }^{33 \mathrm{~b}}$, I.A. Connelly ${ }^{57}$, S. Constantinescu ${ }^{27 b}$, F. Conventi ${ }^{69 a, a w}$, A.M. Cooper-Sarkar ${ }^{135}$, F. Cormier ${ }^{175}$, K.J.R. Cormier ${ }^{167}$, L.D. Corpe ${ }^{94}$, M. Corradi ${ }^{72 a, 72 b}$, E.E. Corrigan ${ }^{96}$,

F. Corriveau ${ }^{103, a c}$, A. Cortes-Gonzalez ${ }^{36}$, M.J. Costa ${ }^{174}$, F. Costanza ${ }^{5}$, D. Costanzo ${ }^{149}$,

G. Cowan $^{93}$, J.W. Cowley ${ }^{32}$, J. Crane ${ }^{100}$, K. Cranmer ${ }^{124}$, S.J. Crawley ${ }^{57}$, R.A. Creager ${ }^{137}$,

S. Crépé-Renaudin ${ }^{58}$, F. Crescioli ${ }^{136}$, M. Cristinziani ${ }^{24}$, V. Croft $^{120}$, G. Crosetti ${ }^{41 b, 41 a}$, A. Cueto $^{5}$,

T. Cuhadar Donszelmann ${ }^{149}$, A.R. Cukierman ${ }^{153}$, S. Czekierda ${ }^{84}$, P. Czodrowski ${ }^{36}$,

M.J. Da Cunha Sargedas De Sousa ${ }^{60 b}$, J.V. Da Fonseca Pinto ${ }^{80 b}$, C. Da Via ${ }^{100}$, W. Dabrowski ${ }^{83 a}$,

T. Dado ${ }^{28 a}$, S. Dahbi ${ }^{35 e}$, T. Dai ${ }^{105}$, C. Dallapiccola ${ }^{102}$, M. Dam ${ }^{40}$, G. D'amen ${ }^{23 b, 23 a}$, J. Damp ${ }^{99}$, J.R. Dandoy ${ }^{137}$, M.F. Daneri ${ }^{30}$, N.P. Dang ${ }^{181}$, N.D Dann ${ }^{100}$, M. Danninger ${ }^{175}$, V. Dao ${ }^{36}$,

G. Darbo ${ }^{55 b}$, O. Dartsi ${ }^{5}$, A. Dattagupta ${ }^{131}$, T. Daubney ${ }^{46}$, S. D'Auria $^{68 a, 68 b}$, W. Davey ${ }^{24}$,

C. David ${ }^{46}$, T. Davidek ${ }^{143}$, D.R. Davis ${ }^{49}$, E. Dawe ${ }^{104}$, I. Dawson ${ }^{149}$, K. De $^{8}$, R. De Asmundis ${ }^{69 a}$,

A. De Benedetti ${ }^{128}$, M. De Beurs ${ }^{120}$, S. De Castro ${ }^{23 b, 23 a}$, S. De Cecco ${ }^{72 a, 72 b}$, N. De Groot ${ }^{119}$,

P. de Jong ${ }^{120}$, H. De la Torre ${ }^{106}$, A. De Maria ${ }^{15 c}$, D. De Pedis ${ }^{72 a}$, A. De Salvo ${ }^{72 a}$,

U. De Sanctis ${ }^{73 a, 73 b}$, M. De Santis ${ }^{73 a, 73 b}$, A. De Santo ${ }^{156}$, K. De Vasconcelos Corga ${ }^{101}$,

J.B. De Vivie De Regie ${ }^{132}$, C. Debenedetti ${ }^{146}$, D.V. Dedovich ${ }^{79}$, A.M. Deiana ${ }^{42}$,

M. Del Gaudio ${ }^{41 \mathrm{~b}, 41 \mathrm{a}}$, J. Del Peso ${ }^{98}$, Y. Delabat Diaz ${ }^{46}$, D. Delgove ${ }^{132}$, F. Deliot ${ }^{145}$,

C.M. Delitzsch ${ }^{7}$, M. Della Pietra ${ }^{69 a, 69 b}$, D. Della Volpe ${ }^{54}$, A. Dell'Acqua ${ }^{36}$, L. Dell'Asta ${ }^{25}$,

M. Delmastro ${ }^{5}$, C. Delporte ${ }^{132}$, P.A. Delsart ${ }^{58}$, D.A. DeMarco ${ }^{167}$, S. Demers ${ }^{183}$, M. Demichev ${ }^{79}$,

G. Demontigny ${ }^{109}$, S.P. Denisov ${ }^{123}$, D. Denysiuk ${ }^{120}$, L. D'Eramo ${ }^{136}$, D. Derendarz ${ }^{84}$,

J.E. Derkaoui ${ }^{35 d}$, F. Derue ${ }^{136}$, P. Dervan ${ }^{90}$, K. Desch ${ }^{24}$, C. Deterre ${ }^{46}$, K. Dette ${ }^{167}$,

M.R. Devesa ${ }^{30}$, P.O. Deviveiros ${ }^{36}$, A. Dewhurst ${ }^{144}$, S. Dhaliwal ${ }^{26}$, F.A. Di Bello ${ }^{54}$,

A. Di Ciaccio ${ }^{73 a, 73 b}$, L. Di Ciaccio ${ }^{5}$, W.K. Di Clemente ${ }^{137}$, C. Di Donato ${ }^{69 a, 69 b}$, A. Di Girolamo ${ }^{36}$,

G. Di Gregorio ${ }^{71 a, 71 b}$, B. Di Micco ${ }^{74 a, 74 b}$, R. Di Nardo ${ }^{102}$, K.F. Di Petrillo ${ }^{59}$, R. Di Sipio ${ }^{167}$,

D. Di Valentino ${ }^{34}$, C. Diaconu ${ }^{101}$, F.A. Dias ${ }^{40}$, T. Dias Do Vale ${ }^{140 a, 140 e}$, M.A. Diaz ${ }^{147 a}$,

J. Dickinson ${ }^{18}$, E.B. Diehl ${ }^{105}$, J. Dietrich ${ }^{19}$, S. Díez Cornell ${ }^{46}$, A. Dimitrievska ${ }^{18}$, W. Ding ${ }^{15 b}$,

J. Dingfelder ${ }^{24}$, F. Dittus ${ }^{36}$, F. Djama ${ }^{101}$, T. Djobava ${ }^{159 b}$, J.I. Djuvsland ${ }^{17}$, M.A.B. Do Vale $^{80 c}$,

M. Dobre ${ }^{27 b}$, D. Dodsworth ${ }^{26}$, C. Doglioni ${ }^{96}$, J. Dolejsi $^{143}$, Z. Dolezal ${ }^{143}$, M. Donadelli $^{80 d}$,

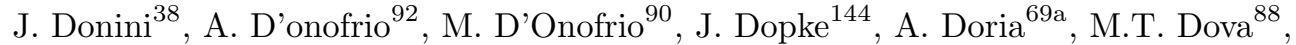

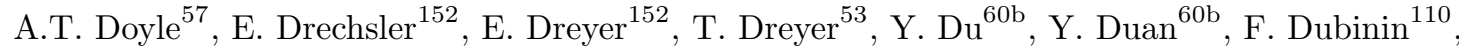

M. Dubovsky ${ }^{28 a}$, A. Dubreuil ${ }^{54}$, E. Duchovni ${ }^{180}$, G. Duckeck $^{114}$, A. Ducourthial ${ }^{136}$,

O.A. Ducu ${ }^{109, w}$, D. Duda ${ }^{115}$, A. Dudarev ${ }^{36}$, A.C. Dudder ${ }^{99}$, E.M. Duffield ${ }^{18}$, L. Duflot ${ }^{132}$,

M. Dührssen ${ }^{36}$, C. Dülsen ${ }^{182}$, M. Dumancic ${ }^{180}$, A.E. Dumitriu ${ }^{27 b}$, A.K. Duncan ${ }^{57}$, M. Dunford ${ }^{61 a}$,

A. Duperrin ${ }^{101}$, H. Duran Yildiz ${ }^{4 a}$, M. Düren ${ }^{56}$, A. Durglishvili ${ }^{159 b}$, D. Duschinger ${ }^{48}$, B. Dutta ${ }^{46}$,

D. Duvnjak ${ }^{1}$, G.I. Dyckes ${ }^{137}$, M. Dyndal ${ }^{46}$, S. Dysch ${ }^{100}$, B.S. Dziedzic ${ }^{84}$, K.M. Ecker ${ }^{115}$,

R.C. Edgar $^{105}$, T. Eifert ${ }^{36}$, G. Eigen $^{17}$, K. Einsweiler ${ }^{18}$, T. Ekelof $^{172}$, M. El Kacimi ${ }^{35 c}$,

R. El Kosseifi ${ }^{101}$, V. Ellajosyula ${ }^{172}$, M. Ellert ${ }^{172}$, F. Ellinghaus ${ }^{182}$, A.A. Elliot $^{92}$, N. Ellis ${ }^{36}$,

J. Elmsheuser ${ }^{29}$, M. Elsing ${ }^{36}$, D. Emeliyanov ${ }^{144}$, A. Emerman $^{39}$, Y. Enari ${ }^{163}$, J.S. Ennis ${ }^{178}$,

M.B. Epland ${ }^{49}$, J. Erdmann ${ }^{47}$, A. Ereditato ${ }^{20}$, M. Escalier ${ }^{132}$, C. Escobar ${ }^{174}$,

O. Estrada Pastor ${ }^{174}$, A.I. Etienvre ${ }^{145}$, E. Etzion ${ }^{161}$, H. Evans ${ }^{65}$, A. Ezhilov ${ }^{138}$, F. Fabbri ${ }^{57}$,

L. Fabbri ${ }^{23 b, 23 a}$, V. Fabiani ${ }^{119}$, G. Facini ${ }^{94}$, R.M. Faisca Rodrigues Pereira ${ }^{140 a}$,

R.M. Fakhrutdinov ${ }^{123}$, S. Falciano ${ }^{72 a}$, P.J. Falke ${ }^{5}$, S. Falke ${ }^{5}$, J. Faltova ${ }^{143}$, Y. Fang ${ }^{15 a}$,

Y. Fang ${ }^{15 a}$, G. Fanourakis ${ }^{44}$, M. Fanti ${ }^{68 a, 68 b}$, A. Farbin ${ }^{8}$, A. Farilla ${ }^{74 a}$, E.M. Farina ${ }^{70 a, 70 b}$,

T. Farooque ${ }^{106}$, S. Farrell ${ }^{18}$, S.M. Farrington ${ }^{178}$, P. Farthouat ${ }^{36}$, F. Fassi ${ }^{35 e}$, P. Fassnacht ${ }^{36}$,

D. Fassouliotis ${ }^{9}$, M. Faucci Giannelli ${ }^{50}$, W.J. Fawcett ${ }^{32}$, L. Fayard ${ }^{132}$, O.L. Fedin ${ }^{138, o}$,

W. Fedorko ${ }^{175}$, M. Feickert ${ }^{42}$, S. Feigl ${ }^{134}$, L. Feligioni ${ }^{101}$, A. Fell ${ }^{149}$, C. Feng ${ }^{60 b}$, E.J. Feng ${ }^{36}$,

M. Feng ${ }^{49}$, M.J. Fenton ${ }^{57}$, A.B. Fenyuk ${ }^{123}$, J. Ferrando ${ }^{46}$, A. Ferrari ${ }^{172}$, P. Ferrari ${ }^{120}$,

R. Ferrari ${ }^{70 a}$, D.E. Ferreira de Lima $^{61 \mathrm{~b}}$, A. Ferrer ${ }^{174}$, D. Ferrere ${ }^{54}$, C. Ferretti ${ }^{105}$, F. Fiedler ${ }^{99}$, 
A. Filipčic ${ }^{91}$, F. Filthaut ${ }^{119}$, K.D. Finelli ${ }^{25}$, M.C.N. Fiolhais ${ }^{140 a}$, L. Fiorini ${ }^{174}$, C. Fischer ${ }^{14}$, F. Fischer ${ }^{114}$, W.C. Fisher ${ }^{106}$, I. Fleck ${ }^{151}$, P. Fleischmann ${ }^{105}$, R.R.M. Fletcher ${ }^{137}$, T. Flick ${ }^{182}$, B.M. Flierl ${ }^{114}$, L.F. Flores ${ }^{137}$, L.R. Flores Castillo ${ }^{63 a}$, F.M. Follega ${ }^{75 a, 75 b}$, N. Fomin ${ }^{17}$, G.T. Forcolin ${ }^{75 a, 75 b}$, A. Formica ${ }^{145}$, F.A. Förster ${ }^{14}$, A.C. Forti ${ }^{100}$, A.G. Foster $^{21}$, D. Fournier ${ }^{132}$, H. Fox ${ }^{89}$, S. Fracchia ${ }^{149}$, P. Francavilla ${ }^{71 a, 71 b}$, M. Franchini ${ }^{23 b, 23 a}$, S. Franchino ${ }^{61 a}$, D. Francis ${ }^{36}$, L. Franconi ${ }^{20}$, M. Franklin ${ }^{59}$, M. Frate ${ }^{171}$, A.N. Fray $^{92}$, B. Freund ${ }^{109}$, W.S. Freund ${ }^{80 b}$, E.M. Freundlich ${ }^{47}$, D.C. Frizzell ${ }^{128}$, D. Froidevaux ${ }^{36}$, J.A. Frost ${ }^{135}$, C. Fukunaga ${ }^{164}$, E. Fullana Torregrosa ${ }^{174}$, E. Fumagalli ${ }^{55 b, 55 a}$, T. Fusayasu ${ }^{116}$, J. Fuster ${ }^{174}$, A. Gabrielli ${ }^{23 b, 23 a}$, A. Gabrielli ${ }^{18}$, G.P. Gach ${ }^{83 a}$, S. Gadatsch ${ }^{54}$, P. Gadow ${ }^{115}$, G. Gagliardi ${ }^{55 b}$,55a , L.G. Gagnon ${ }^{109}$, C. Galea ${ }^{27 b}$, B. Galhardo ${ }^{140 a, 140 c}$, E.J. Gallas ${ }^{135}$, B.J. Gallop ${ }^{144}$, P. Gallus ${ }^{142}$, G. Galster ${ }^{40}$, R. Gamboa Goni ${ }^{92}$, K.K. Gan ${ }^{126}$, S. Ganguly ${ }^{180}$, J. Gao ${ }^{60 a}$, Y. Gao ${ }^{90}$, Y.S. Gao ${ }^{31,1}$, C. García ${ }^{174}$, J.E. García Navarro ${ }^{174}$, J.A. García Pascual ${ }^{15 a}$, C. Garcia-Argos ${ }^{52}$, M. Garcia-Sciveres ${ }^{18}$, R.W. Gardner ${ }^{37}$, N. Garelli ${ }^{153}$, S. Gargiulo ${ }^{52}$, V. Garonne ${ }^{134}$, A. Gaudiello ${ }^{55 b, 55 a}$, G. Gaudio ${ }^{70 a}$, I.L. Gavrilenko ${ }^{110}$, A. Gavrilyuk ${ }^{111}$, C. Gay ${ }^{175}$, G. Gaycken ${ }^{24}$, E.N. Gazis ${ }^{10}$, A.A. Geanta ${ }^{27 b}$, C.N.P. Gee ${ }^{144}$, J. Geisen ${ }^{53}$, M. Geisen ${ }^{99}$, M.P. Geisler ${ }^{61 \mathrm{a}}$, C. Gemme ${ }^{55 \mathrm{~b}}$, M.H. Genest ${ }^{58}$, C. Geng ${ }^{105}$, S. Gentile ${ }^{72 a, 72 b}$, S. George ${ }^{93}$, T. Geralis ${ }^{44}$, D. Gerbaudo ${ }^{14}$, L.O. Gerlach ${ }^{53}$, G. Gessner ${ }^{47}$, S. Ghasemi ${ }^{151}$, M. Ghasemi Bostanabad ${ }^{176}$, M. Ghneimat ${ }^{24}$, A. Ghosh ${ }^{77}$, B. Giacobbe ${ }^{23 b}$, S. Giagu ${ }^{72 a, 72 b}$, N. Giangiacomi ${ }^{23 b, 23 a}$, P. Giannetti ${ }^{71 a}$, A. Giannini ${ }^{69 a, 69 b}$, S.M. Gibson ${ }^{93}$, M. Gignac ${ }^{146}$, D. Gillberg ${ }^{34}$, G. Gilles ${ }^{182}$, D.M. Gingrich ${ }^{3, a v}$, M.P. Giordani ${ }^{66 a, 66 c}$, F.M. Giorgi ${ }^{23 b}$, P.F. Giraud ${ }^{145}$, G. Giugliarelli ${ }^{66 a, 66 c}$, D. Giugni ${ }^{68 a}$, F. Giuli ${ }^{73 a, 73 b}$, M. Giulini ${ }^{61 b}$, S. Gkaitatzis ${ }^{162}$, I. Gkialas ${ }^{9}$ h, E.L. Gkougkousis ${ }^{14}$, P. Gkountoumis ${ }^{10}$, L.K. Gladilin ${ }^{113}$, C. Glasman ${ }^{98}$, J. Glatzer ${ }^{14}$, P.C.F. Glaysher ${ }^{46}$, A. Glazov ${ }^{46}$, M. Goblirsch-Kolb ${ }^{26}$, S. Goldfarb ${ }^{104}$, T. Golling ${ }^{54}$, D. Golubkov ${ }^{123}$, A. Gomes ${ }^{140 a, 140 b}$, R. Goncalves Gama ${ }^{53}$, R. Gonçalo ${ }^{140 a, 140 b}$, G. Gonella ${ }^{52}$, L. Gonella ${ }^{21}$, A. Gongadze ${ }^{79}$, F. Gonnella ${ }^{21}$, J.L. Gonski ${ }^{59}$,

S. González de la $\mathrm{Hoz}^{174}$, S. Gonzalez-Sevilla ${ }^{54}$, G.R. Gonzalvo Rodriguez ${ }^{174}$, L. Goossens ${ }^{36}$, P.A. Gorbounov ${ }^{111}$, H.A. Gordon ${ }^{29}$, B. Gorini ${ }^{36}$, E. Gorini ${ }^{67 a, 67 b}$, A. Gorišek ${ }^{91}$, A.T. Goshaw ${ }^{49}$, C. Gössling ${ }^{47}$, M.I. Gostkin ${ }^{79}$, C.A. Gottardo ${ }^{24}$, C.R. Goudet ${ }^{132}$, M. Gouighri ${ }^{35 b}$,

D. Goujdami ${ }^{35 c}$, A.G. Goussiou ${ }^{148}$, N. Govender ${ }^{33 b, a}$, C. Goy $^{5}$, E. Gozani ${ }^{160}$,

I. Grabowska-Bold ${ }^{83 a}$, P.O.J. Gradin ${ }^{172}$, E.C. Graham ${ }^{90}$, J. Gramling ${ }^{171}$, E. Gramstad ${ }^{134}$, S. Grancagnolo ${ }^{19}$, M. Grandi ${ }^{156}$, V. Gratchev ${ }^{138}$, P.M. Gravila ${ }^{27 f}$, F.G. Gravili ${ }^{67 a, 67 b}$, C. Gray $^{57}$, H.M. Gray ${ }^{18}$, C. Grefe ${ }^{24}$, K. Gregersen ${ }^{96}$, I.M. Gregor ${ }^{46}$, P. Grenier ${ }^{153}$, K. Grevtsov ${ }^{46}$, N.A. Grieser ${ }^{128}$, J. Griffiths ${ }^{8}$, A.A. Grillo ${ }^{146}$, K. Grimm ${ }^{31, k}$, S. Grinstein ${ }^{14, x}$, J.-F. Grivaz ${ }^{132}$, S. Groh ${ }^{99}$, E. Gross ${ }^{180}$, J. Grosse-Knetter ${ }^{53}$, Z.J. Grout ${ }^{94}$, C. Grud ${ }^{105}$, A. Grummer ${ }^{118}$, L. Guan ${ }^{105}$, W. Guan ${ }^{181}$, J. Guenther ${ }^{36}$, A. Guerguichon ${ }^{132}$, F. Guescini ${ }^{168 a}$, D. Guest ${ }^{171}$,

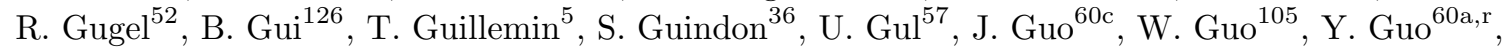
Z. Guo ${ }^{101}$, R. Gupta ${ }^{46}$, S. Gurbuz ${ }^{12 c}$, G. Gustavino ${ }^{128}$, P. Gutierrez ${ }^{128}$, C. Gutschow ${ }^{94}$, C. Guyot ${ }^{145}$, M.P. Guzik ${ }^{83 a}$, C. Gwenlan ${ }^{135}$, C.B. Gwilliam ${ }^{90}$, A. Haas ${ }^{124}$, C. Haber ${ }^{18}$,

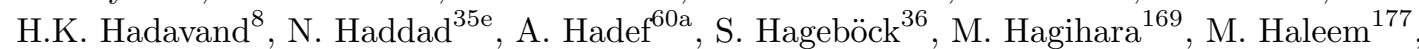
J. Haley ${ }^{129}$, G. Halladjian ${ }^{106}$, G.D. Hallewell ${ }^{101}$, K. Hamacher ${ }^{182}$, P. Hamal ${ }^{130}$, K. Hamano ${ }^{176}$, H. Hamdaoui ${ }^{35 e}$, G.N. Hamity ${ }^{149}$, K. Han ${ }^{60 a, a j}$, L. $\operatorname{Han}^{60 a}$, S. Han ${ }^{15 a, 15 d}$, K. Hanagaki ${ }^{81, u}$, M. Hance ${ }^{146}$, D.M. Handl ${ }^{114}$, B. Haney ${ }^{137}$, R. Hankache ${ }^{136}$, P. Hanke ${ }^{61 a}$, E. Hansen ${ }^{96}$, J.B. Hansen ${ }^{40}$, J.D. Hansen ${ }^{40}$, M.C. Hansen ${ }^{24}$, P.H. Hansen ${ }^{40}$, E.C. Hanson ${ }^{100}$, K. Hara ${ }^{169}$, A.S. Hard ${ }^{181}$, T. Harenberg ${ }^{182}$, S. Harkusha ${ }^{107}$, P.F. Harrison ${ }^{178}$, N.M. Hartmann ${ }^{114}$, Y. Hasegawa ${ }^{150}$, A. Hasib ${ }^{50}$, S. Hassani ${ }^{145}$, S. $\operatorname{Haug}^{20}$, R. Hauser ${ }^{106}$, L. Hauswald ${ }^{48}$, L.B. Havener ${ }^{39}$, M. Havranek ${ }^{142}$, C.M. Hawkes ${ }^{21}$, R.J. Hawkings ${ }^{36}$, D. Hayden ${ }^{106}$, C. Hayes ${ }^{155}$, R.L. Hayes ${ }^{175}$, C.P. Hays ${ }^{135}$, J.M. Hays ${ }^{92}$, H.S. Hayward ${ }^{90}$, S.J. Haywood ${ }^{144}$, F. He ${ }^{60 a}$,

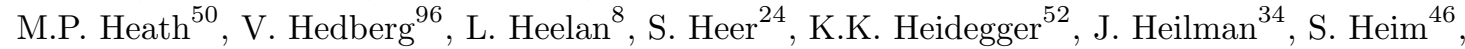
T. Heim ${ }^{18}$, B. Heinemann ${ }^{46, a q}$, J.J. Heinrich ${ }^{131}$, L. Heinrich ${ }^{36}$, C. Heinz ${ }^{56}$, J. Hejbal ${ }^{141}$, 
L. Helary ${ }^{61 \mathrm{~b}}$, A. Held ${ }^{175}$, S. Hellesund ${ }^{134}$, C.M. Helling ${ }^{146}$, S. Hellman ${ }^{45 a, 45 b}$, C. Helsens ${ }^{36}$, R.C.W. Henderson ${ }^{89}$, Y. Heng ${ }^{181}$, S. Henkelmann ${ }^{175}$, A.M. Henriques Correia ${ }^{36}$, G.H. Herbert ${ }^{19}$, H. $\operatorname{Herde}^{26}$, V. Herget ${ }^{177}$, Y. Hernández Jiménez ${ }^{33 c}$, H. Herr ${ }^{99}$, M.G. Herrmann ${ }^{114}$,

T. Herrmann ${ }^{48}$, G. Herten ${ }^{52}$, R. Hertenberger ${ }^{114}$, L. Hervas ${ }^{36}$, T.C. Herwig ${ }^{137}$, G.G. Hesketh ${ }^{94}$, N.P. Hessey ${ }^{168 a}$, A. Higashida ${ }^{163}$, S. Higashino ${ }^{81}$, E. Higón-Rodriguez ${ }^{174}$, K. Hildebrand ${ }^{37}$, E. Hill ${ }^{176}$, J.C. Hill ${ }^{32}$, K.K. Hill ${ }^{29}$, K.H. Hiller ${ }^{46}$, S.J. Hillier ${ }^{21}$, M. Hils ${ }^{48}$, I. Hinchliffe ${ }^{18}$, F. Hinterkeuser ${ }^{24}$, M. Hirose ${ }^{133}$, S. Hirose ${ }^{52}$, D. Hirschbuehl ${ }^{182}$, B. Hiti ${ }^{91}$, O. Hladik ${ }^{141}$, D.R. Hlaluku ${ }^{33 c}$, X. Hoad ${ }^{50}$, J. Hobbs ${ }^{155}$, N. Hod ${ }^{180}$, M.C. Hodgkinson ${ }^{149}$, A. Hoecker ${ }^{36}$, F. Hoenig ${ }^{114}$, D. Hohn ${ }^{52}$, D. Hohov ${ }^{132}$, T.R. Holmes ${ }^{37}$, M. Holzbock ${ }^{114}$, L.B.A.H Hommels ${ }^{32}$, S. Honda ${ }^{169}$, T. Honda ${ }^{81}$, T.M. Hong ${ }^{139}$, A. Hönle ${ }^{115}$, B.H. Hooberman ${ }^{173}$, W.H. Hopkins ${ }^{6}$,

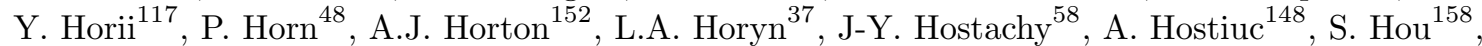

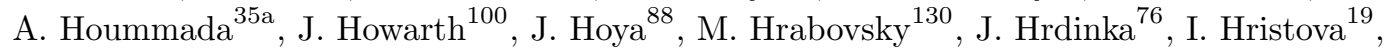
J. Hrivnac ${ }^{132}$, A. Hrynevich ${ }^{108}$, T. Hryn'ova ${ }^{5}$, P.J. $\mathrm{Hsu}^{64}$, S.-C. $\mathrm{Hsu}^{148}$, Q. $\mathrm{Hu}^{29}$, S. Hu ${ }^{60 c}$, Y. Huang ${ }^{15 a}$, Z. Hubacek ${ }^{142}$, F. Hubaut ${ }^{101}$, M. Huebner ${ }^{24}$, F. Huegging ${ }^{24}$, T.B. Huffman ${ }^{135}$, M. Huhtinen ${ }^{36}$, R.F.H. Hunter ${ }^{34}$, P. Huo ${ }^{155}$, A.M. Hupe ${ }^{34}$, N. Huseynov ${ }^{79}$ ae, J. Huston ${ }^{106}$,

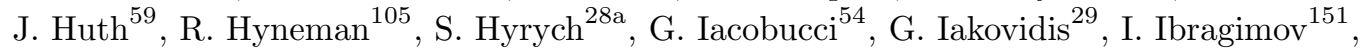
L. Iconomidou-Fayard ${ }^{132}$, Z. Idrissi ${ }^{35 e}$, P.I. Iengo ${ }^{36}$, R. Ignazzi ${ }^{40}$, O. Igonkina ${ }^{120, z}$, R. Iguchi ${ }^{163}$,

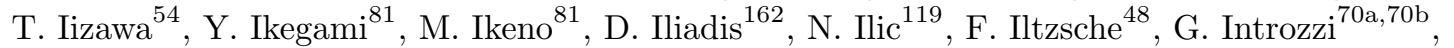

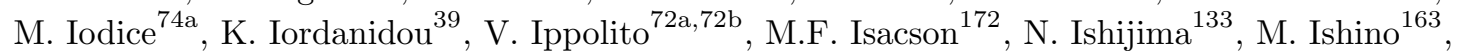

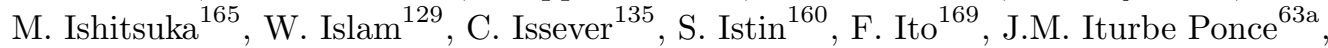
R. Iuppa ${ }^{75 a, 75 b}$, A. Ivina ${ }^{180}$, H. Iwasaki ${ }^{81},{\text { J.M. } \text { Izen }^{43}, \text { V. Izzo }}^{69 a}$, P. Jacka ${ }^{141}$, P. Jackson ${ }^{1}$, R.M. Jacobs ${ }^{24}$, V. Jain ${ }^{2}$, G. Jäkel ${ }^{182}$, K.B. Jakobi ${ }^{99}$, K. Jakobs ${ }^{52}$, S. Jakobsen ${ }^{76}$, T. Jakoubek ${ }^{141}$, J. Jamieson ${ }^{57}$, D.O. Jamin ${ }^{129}$, R. Jansky ${ }^{54}$, J. Janssen ${ }^{24}$, M. Janus ${ }^{53}$, P.A. Janus ${ }^{83 a}$, G. Jarlskog ${ }^{96}$, N. Javadov ${ }^{79, a e}$, T. Javưrek ${ }^{36}$, M. Javurkova ${ }^{52}$, F. Jeanneau ${ }^{145}$, L. Jeanty ${ }^{131}$ J. Jejelava ${ }^{159 a, a f}$, A. Jelinskas ${ }^{178}$, P. Jenni ${ }^{52, b}$, J. Jeong ${ }^{46}$, N. Jeong ${ }^{46}$, S. Jézéquel ${ }^{5}$, H. Ji ${ }^{181}$, J. Jia ${ }^{155}$, H. Jiang ${ }^{78}$, Y. Jiang ${ }^{60 a}$, Z. Jiang ${ }^{153, \mathrm{p}}$, S. Jiggins ${ }^{52}$, F.A. Jimenez Morales ${ }^{38}$, J. Jimenez Pena ${ }^{174}$, S. Jin ${ }^{15 c}$, A. Jinaru ${ }^{27 b}$, O. Jinnouchi ${ }^{165}$, H. Jivan ${ }^{33 c}$, P. Johansson ${ }^{149}$, K.A. Johns ${ }^{7}$, C.A. Johnson ${ }^{65}$, K. Jon-And ${ }^{45 a, 45 b}$, R.W.L. Jones ${ }^{89}$, S.D. Jones ${ }^{156}$, S. Jones ${ }^{7}$, T.J. Jones ${ }^{90}$, J. Jongmanns ${ }^{61 a}$, P.M. Jorge ${ }^{140 a, 140 b}$, J. Jovicevic ${ }^{168 a}$, X. Ju ${ }^{18}$, J.J. Junggeburth ${ }^{115}$, A. Juste Rozas ${ }^{14, \mathrm{x}}$, A. Kaczmarska ${ }^{84}$, M. Kado ${ }^{132}$, H. $\operatorname{Kagan}^{126}$, M. Kagan ${ }^{153}$, T. Kaji ${ }^{179}$, E. Kajomovitz ${ }^{160}$, C.W. Kalderon ${ }^{96}$, A. Kaluza ${ }^{99}$, A. Kamenshchikov ${ }^{123}$, L. Kanjir ${ }^{91}$, Y. Kano ${ }^{163}$, V.A. Kantserov ${ }^{112}$, J. Kanzaki ${ }^{81}$, L.S. Kaplan ${ }^{181}$, D. $\operatorname{Kar}^{33 c}$, M.J. Kareem ${ }^{168 b}$, E. Karentzos ${ }^{10}$, S.N. Karpov ${ }^{79}$, Z.M. Karpova ${ }^{79}$, V. Kartvelishvili ${ }^{89}$, A.N. Karyukhin ${ }^{123}$, L. Kashif ${ }^{181}$, R.D. Kass ${ }^{126}$, A. Kastanas ${ }^{45 a, 45 b}$, Y. Kataoka ${ }^{163}$, C. Kato ${ }^{60 d, 60 c}$, J. Katzy ${ }^{46}$, K. Kawade ${ }^{82}$, K. Kawagoe ${ }^{87}$, T. Kawaguchi ${ }^{117}$, T. Kawamoto ${ }^{163}$, G. Kawamura ${ }^{53}$, E.F. Kay ${ }^{176}$, V.F. Kazanin ${ }^{122 b, 122 a}$, R. Keeler ${ }^{176}$, R. Kehoe ${ }^{42}$, J.S. Keller ${ }^{34}$, E. Kellermann ${ }^{96}$, J.J. Kempster ${ }^{21}$, J. Kendrick ${ }^{21}$, O. Kepka ${ }^{141}$, S. Kersten ${ }^{182}$, B.P. Kerševan ${ }^{91}$, S. Ketabchi Haghighat ${ }^{167}$, R.A. Keyes ${ }^{103}$, M. Khader ${ }^{173}$, F. Khalil-Zada ${ }^{13}$, A. Khanov ${ }^{129}$, A.G. Kharlamov ${ }^{122 b, 122 a}$, T. Kharlamova ${ }^{122 b, 122 a}$, E.E. Khoda ${ }^{175}$, A. Khodinov ${ }^{166}$, T.J. Khoo ${ }^{54}$, E. $\operatorname{Khramov}^{79}$, J. Khubua ${ }^{159 b}$, S. Kido ${ }^{82}$, M. Kiehn ${ }^{54}$, C.R. Kilby ${ }^{93}$, Y.K. Kim ${ }^{37}$, N. Kimura ${ }^{66 a, 66 c}$, O.M. Kind ${ }^{19}$, B.T. King ${ }^{90, *}$, D. Kirchmeier ${ }^{48}$, J. Kirk ${ }^{144}$, A.E. Kiryunin ${ }^{115}$, T. Kishimoto ${ }^{163}$, V. Kitali ${ }^{46}$, O. Kivernyk $^{5}$, E. Kladiva ${ }^{28 b, *}$, T. Klapdor-Kleingrothaus ${ }^{52}$, M.H. Klein ${ }^{105}$, M. Klein ${ }^{90}$, U. Klein ${ }^{90}$, K. Kleinknecht ${ }^{99}$, P. Klimek ${ }^{121}$, A. Klimentov ${ }^{29}$, T. Klingl ${ }^{24}$, T. Klioutchnikova ${ }^{36}$, F.F. Klitzner ${ }^{114}$, P. Kluit ${ }^{120}$, S. Kluth ${ }^{115}$, E. Kneringer ${ }^{76}$, E.B.F.G. Knoops ${ }^{101}$, A. Knue ${ }^{52}$, D. Kobayashi ${ }^{87}$, T. Kobayashi ${ }^{163}$, M. Kobel ${ }^{48}$, M. Kocian ${ }^{153}$, P. Kodys ${ }^{143}$, P.T. Koenig ${ }^{24}$, T. Koffas ${ }^{34}$, N.M. Köhler ${ }^{115}$, T. Koi ${ }^{153}$, M. Kolb ${ }^{61 b}$, I. Koletsou ${ }^{5}$, T. Kondo ${ }^{81}$, N. Kondrashova ${ }^{60 c}$, K. Köneke ${ }^{52}$, A.C. König ${ }^{119}$, T. Kono ${ }^{125}$, R. Konoplich ${ }^{124, a m}$, V. Konstantinides ${ }^{94}$, N. Konstantinidis ${ }^{94}$, B. Konya ${ }^{96}$, R. Kopeliansky ${ }^{65}$, S. Koperny ${ }^{83 a}$, 


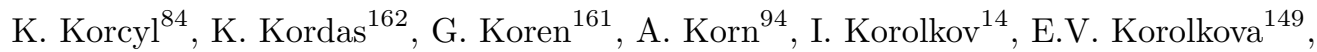
N. Korotkova ${ }^{113}$, O. Kortner ${ }^{115}$, S. Kortner ${ }^{115}$, T. Kosek ${ }^{143}$, V.V. Kostyukhin ${ }^{24}$, A. Kotwal ${ }^{49}$, A. Koulouris ${ }^{10}$, A. Kourkoumeli-Charalampidi ${ }^{70 a, 70 b}$, C. Kourkoumelis ${ }^{9}$, E. Kourlitis ${ }^{149}$, V. Kouskoura ${ }^{29}$, A.B. Kowalewska ${ }^{84}$, R. Kowalewski ${ }^{176}$, C. Kozakai ${ }^{163}$, W. Kozanecki ${ }^{145}$, A.S. Kozhin ${ }^{123}$, V.A. Kramarenko ${ }^{113}$, G. Kramberger ${ }^{91}$, D. Krasnopevtsev ${ }^{60 a}$, M.W. Krasny ${ }^{136}$, A. Krasznahorkay ${ }^{36}$, D. Krauss ${ }^{115}$, J.A. Kremer ${ }^{83 a}$, J. Kretzschmar ${ }^{90}$, P. Krieger ${ }^{167}$,

A. Krishnan ${ }^{61 b}$, K. Krizka ${ }^{18}$, K. Kroeninger ${ }^{47}$, H. Kroha ${ }^{115}$, J. Kroll ${ }^{141}$, J. Kroll ${ }^{137}$, J. Krstic ${ }^{16}$, U. Kruchonak $^{79}$, H. Krüger ${ }^{24}$, N. Krumnack ${ }^{78}$, M.C. Kruse ${ }^{49}$, T. Kubota ${ }^{104}$, S. Kuday ${ }^{4 b}$, J.T. Kuechler ${ }^{46}$, S. Kuehn ${ }^{36}$, A. Kugel ${ }^{61 \text { a }}$, T. Kuhl ${ }^{46}$, V. Kukhtin ${ }^{79}$, R. Kukla ${ }^{101}$, Y. Kulchitsky ${ }^{107, \text { ai }}$, S. Kuleshov ${ }^{147 b}$, Y.P. Kulinich ${ }^{173}$, M. Kuna ${ }^{58}$, T. Kunigo ${ }^{85}$, A. Kupco ${ }^{141}$ T. Kupfer ${ }^{47}$, O. Kuprash ${ }^{52}$, H. Kurashige ${ }^{82}$, L.L. Kurchaninov ${ }^{168 a}$, Y.A. Kurochkin ${ }^{107}$ A. Kurova ${ }^{112}$, M.G. Kurth ${ }^{15 a, 15 d}$, E.S. Kuwertz ${ }^{36}$, M. Kuze ${ }^{165}$, A.K. Kvam ${ }^{148}$, J. Kvita ${ }^{130}$, T. Kwan ${ }^{103}$, A. La Rosa ${ }^{115}$, J.L. La Rosa Navarro ${ }^{80 d}$, L. La Rotonda ${ }^{41 b, 41 a}$, F. La Ruffa ${ }^{41 b, 41 a}$, C. Lacasta $^{174}$, F. Lacava ${ }^{72 a, 72 b}$, D.P.J. Lack ${ }^{100}$, H. Lacker ${ }^{19}$, D. Lacour ${ }^{136}$, E. Ladygin ${ }^{79}$,

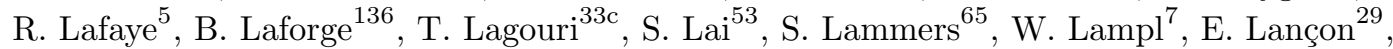
U. Landgraf $^{52}$, M.P.J. Landon ${ }^{92}$, M.C. Lanfermann ${ }^{54}$, V.S. Lang ${ }^{46}$, J.C. Lange ${ }^{53}$,

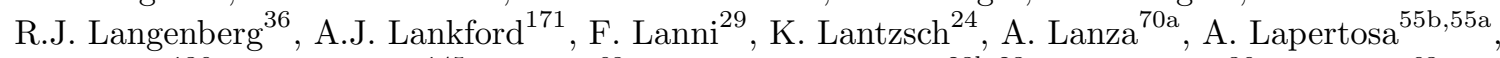
S. Laplace ${ }^{136}$, J.F. Laporte ${ }^{145}$, T. Lari ${ }^{68 a}$, F. Lasagni Manghi ${ }^{23 b, 23 a}$, M. Lassnig ${ }^{36}$, T.S. Lau ${ }^{63 a}$,

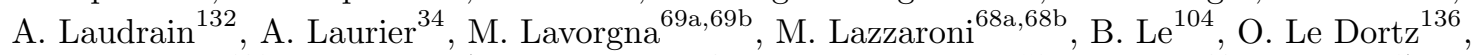
E. Le Guirriec ${ }^{101}$, M. LeBlanc ${ }^{7}$, T. LeCompte ${ }^{6}$, F. Ledroit-Guillon ${ }^{58}$, C.A. Lee ${ }^{29}$, G.R. Lee ${ }^{17}$, L. Lee $^{59}$, S.C. Lee ${ }^{158}$, S.J. Lee ${ }^{34}$, B. Lefebvre ${ }^{168 a}$, M. Lefebvre ${ }^{176}$, F. Legger ${ }^{14}$, C. Leggett ${ }^{18}$, K. Lehmann ${ }^{152}$, N. Lehmann ${ }^{182}$, G. Lehmann Miotto ${ }^{36}$, W.A. Leight ${ }^{46}$, A. Leisos ${ }^{162, v}$, M.A.L. Leite ${ }^{80 d}$, R. Leitner ${ }^{143}$, D. Lellouch ${ }^{180, *}$, K.J.C. Leney ${ }^{42}$, T. Lenz ${ }^{24}$, B. Lenzi ${ }^{36}$, R. Leone $^{7}$, S. Leone ${ }^{71 a}$, C. Leonidopoulos ${ }^{50}$, A. Leopold ${ }^{136}$, G. Lerner ${ }^{156}$, C. Leroy ${ }^{109}$, R. Les ${ }^{167}$, C.G. Lester ${ }^{32}$, M. Levchenko ${ }^{138}$, J. Levêque ${ }^{5}$, D. Levin ${ }^{105}$, L.J. Levinson ${ }^{180}$, D.J. Lewis ${ }^{21}$,

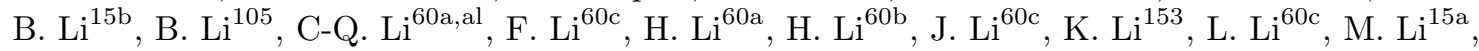

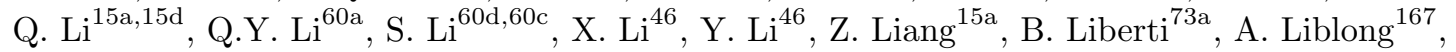
K. Lie ${ }^{63 c}$, S. Liem ${ }^{120}$, C.Y. $\operatorname{Lin}^{32}$, K. $\operatorname{Lin}^{106}$, T.H. Lin ${ }^{99}$, R.A. Linck ${ }^{65}$, J.H. Lindon ${ }^{21}$, A.L. Lionti ${ }^{54}$, E. Lipeles ${ }^{137}$, A. Lipniacka ${ }^{17}$, M. Lisovyi ${ }^{61 b}$, T.M. Liss ${ }^{173 \text {,as }}$, A. Lister ${ }^{175}$, A.M. Litke ${ }^{146}$, J.D. Little ${ }^{8}$, B. Liu ${ }^{78}$, B.L Liu ${ }^{6}$, H.B. Liu ${ }^{29}$, H. Liu ${ }^{105}$, J.B. Liu ${ }^{60 a}$, J.K.K. Liu ${ }^{135}$,

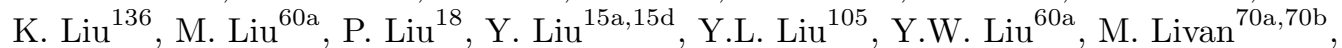
A. Lleres $^{58}$, J. Llorente Merino ${ }^{15 a}$, S.L. Lloyd ${ }^{92}$, C.Y. Lo ${ }^{63 b}$, F. Lo Sterzo ${ }^{42}$, E.M. Lobodzinska ${ }^{46}$,

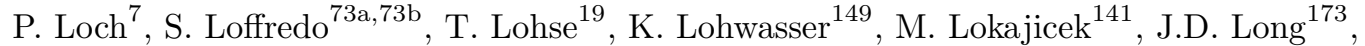
R.E. Long $^{89}$, L. Longo ${ }^{36}$, K.A. Looper ${ }^{126}$, J.A. Lopez ${ }^{147 b}$, I. Lopez Paz ${ }^{100}$, A. Lopez Solis ${ }^{149}$ J. Lorenz ${ }^{114}$, N. Lorenzo Martinez ${ }^{5}$, M. Losada ${ }^{22}$, P.J. Lösel ${ }^{114}$, A. Lösle ${ }^{52}$, X. Lou ${ }^{46}$, X. Lou ${ }^{15 a}$,

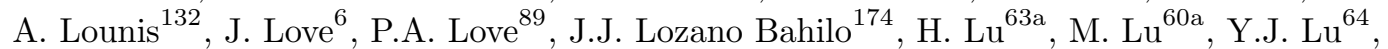
H.J. Lubatti ${ }^{148}$, C. Luci ${ }^{72 a, 72 b}$, A. Lucotte ${ }^{58}$, C. Luedtke ${ }^{52}$, F. Luehring ${ }^{65}$, I. Luise ${ }^{136}$, L. Luminari ${ }^{72 a}$, B. Lund-Jensen ${ }^{154}$, M.S. Lutz ${ }^{102}$, D. Lynn ${ }^{29}$, R. Lysak ${ }^{141}$, E. Lytken ${ }^{96}$, F. Lyu ${ }^{15 a}$, V. Lyubushkin ${ }^{79}$, T. Lyubushkina ${ }^{79}$, H. $\mathrm{Ma}^{29}$, L.L. Ma ${ }^{60 \mathrm{~b}}$, Y. Ma ${ }^{60 \mathrm{~b}}$, G. Maccarrone ${ }^{51}$, A. Macchiolo ${ }^{115}$, C.M. Macdonald ${ }^{149}$, J. Machado Miguens ${ }^{137,140 b}$, D. Madaffari ${ }^{174}$, R. Madar ${ }^{38}$, W.F. Mader ${ }^{48}$, N. Madysa ${ }^{48}$, J. Maeda ${ }^{82}$, K. Maekawa ${ }^{163}$, S. Maeland ${ }^{17}$, T. Maeno ${ }^{29}$, M. Maerker ${ }^{48}$, A.S. Maevskiy ${ }^{113}$, V. Magerl ${ }^{52}$, N. Magini ${ }^{78}$, D.J. Mahon ${ }^{39}$, C. Maidantchik ${ }^{80 b}$ T. Maier $^{114}$, A. Maio 140a,140b,140d , O. Majersky ${ }^{28 a}$, S. Majewski ${ }^{131}$, Y. Makida ${ }^{81}$, N. Makovec ${ }^{132}$, B. Malaescu ${ }^{136}$, Pa. Malecki ${ }^{84}$, V.P. Maleev ${ }^{138}$, F. Malek ${ }^{58}$, U. Mallik ${ }^{77}$, D. Malon ${ }^{6}$, C. Malone ${ }^{32}$, S. Maltezos ${ }^{10}$, S. Malyukov ${ }^{36}$, J. Mamuzic ${ }^{174}$, G. Mancini ${ }^{51}$, I. Mandić ${ }^{91}$,

L. Manhaes de Andrade Filho ${ }^{80 a}$, I.M. Maniatis ${ }^{162}$, J. Manjarres Ramos ${ }^{48}$, K.H. Mankinen ${ }^{96}$, A. Mann $^{114}$, A. Manousos ${ }^{76}$, B. Mansoulie ${ }^{145}$, I. Manthos ${ }^{162}$, S. Manzoni ${ }^{120}$, A. Marantis ${ }^{162}$, G. Marceca ${ }^{30}$, L. Marchese ${ }^{135}$, G. Marchiori ${ }^{136}$, M. Marcisovsky ${ }^{141}$, C. Marcon ${ }^{96}$, 
C.A. Marin Tobon ${ }^{36}$, M. Marjanovic ${ }^{38}$, F. Marroquim ${ }^{80 b}$, Z. Marshall ${ }^{18}$, M.U.F Martensson ${ }^{172}$, S. Marti-Garcia ${ }^{174}$, C.B. Martin ${ }^{126}$, T.A. Martin ${ }^{178}$, V.J. Martin ${ }^{50}$, B. Martin dit Latour ${ }^{17}$, M. Martinez ${ }^{14, \mathrm{x}}$, V.I. Martinez Outschoorn ${ }^{102}$, S. Martin-Haugh ${ }^{144}$, V.S. Martoiu ${ }^{27 \mathrm{~b}}$, A.C. Martyniuk ${ }^{94}$, A. Marzin ${ }^{36}$, L. Masetti ${ }^{99}$, T. Mashimo ${ }^{163}$, R. Mashinistov ${ }^{110}$, J. Masik ${ }^{100}$, A.L. Maslennikov ${ }^{122 b, 122 a}$, L.H. Mason ${ }^{104}$, L. Massa ${ }^{73 a, 73 b}$, P. Massarotti ${ }^{69 a, 69 b}$, P. Mastrandrea ${ }^{71 a, 71 b}$, A. Mastroberardino ${ }^{41 b, 41 a}$, T. Masubuchi ${ }^{163}$, A. Matic ${ }^{114}$, P. Mättig ${ }^{24}$, J. Maurer ${ }^{27 b}$, B. Maček ${ }^{91}$, S.J. Maxfield ${ }^{90}$, D.A. Maximov ${ }^{122 b, 122 a}$, R. Mazini ${ }^{158}$, I. Maznas ${ }^{162}$, S.M. Mazza ${ }^{146}$, S.P. Mc Kee ${ }^{105}$, T.G. McCarthy ${ }^{115}$, L.I. McClymont ${ }^{94}$, W.P. McCormack ${ }^{18}$, E.F. McDonald ${ }^{104}$, J.A. Mcfayden ${ }^{36}$, M.A. McKay ${ }^{42}$, K.D. McLean ${ }^{176}$, S.J. McMahon ${ }^{144}$, P.C. $\mathrm{McNamara}^{104}$, C.J. $\mathrm{McNicol}^{178}$, R.A. $\mathrm{McPherson}^{176, \text { ac }}$, J.E. Mdhluli ${ }^{33 \mathrm{c}}$, Z.A. Meadows ${ }^{102}$,

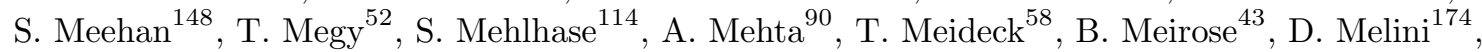
B.R. Mellado Garcia ${ }^{33 c}$, J.D. Mellenthin ${ }^{53}$, M. Melo ${ }^{28 a}$, F. Meloni ${ }^{46}$, A. Melzer ${ }^{24}$, S.B. Menary ${ }^{100}$, E.D. Mendes Gouveia ${ }^{140 a, 140 e}$, L. Meng ${ }^{36}$, X.T. Meng ${ }^{105}$, S. Menke ${ }^{115}$, E. Meoni ${ }^{41 b, 41 a}$, S. Mergelmeyer ${ }^{19}$, S.A.M. Merkt ${ }^{139}$, C. Merlassino ${ }^{20}$, P. Mermod ${ }^{54}$, L. Merola $^{69 a, 69 b}$, C. Meroni ${ }^{6 \mathrm{a}}$, O. Meshkov ${ }^{113}$, J.K.R. Meshreki ${ }^{151}$, A. Messina ${ }^{72 a, 72 b}$, J. Metcalfe $^{6}$, A.S. Mete ${ }^{171}$, C. Meyer ${ }^{65}$, J. Meyer ${ }^{160}$, J-P. Meyer ${ }^{145}$, H. Meyer Zu Theenhausen ${ }^{61 \mathrm{a}}$, F. Miano ${ }^{156}$, R.P. Middleton ${ }^{144}$, L. Mijović ${ }^{50}$, G. Mikenberg ${ }^{180}$, M. Mikestikova ${ }^{141}$, M. Mikuž ${ }^{91}$, H. Mildner $^{149}$, M. Milesi ${ }^{104}$, A. Milic ${ }^{167}$, D.A. Millar ${ }^{92}$, D.W. Miller ${ }^{37}$, A. Milov ${ }^{180}$, D.A. Milstead ${ }^{45 a, 45 b}$, R.A. Mina ${ }^{153, \mathrm{p}}$, A.A. Minaenko ${ }^{123}$, M. Miñano Moya ${ }^{174}$, I.A. Minashvili ${ }^{159 \mathrm{~b}}$, A.I. Mincer ${ }^{124}$, B. Mindur ${ }^{83 a}$, M. Mineev ${ }^{79}$, Y. Minegishi ${ }^{163}$, Y. Ming ${ }^{181}$, L.M. Mir ${ }^{14}$, A. Mirto ${ }^{67 a, 67 b}$, K.P. Mistry ${ }^{137}$, T. Mitani ${ }^{179}$, J. Mitrevski ${ }^{114}$, V.A. Mitsou ${ }^{174}$, M. Mittal ${ }^{60 c}$, A. Miucci ${ }^{20}$, P.S. Miyagawa ${ }^{149}$, A. Mizukami ${ }^{81}$, J.U. Mjörnmark ${ }^{96}$, T. Mkrtchyan $^{184}$, M. Mlynarikova ${ }^{143}$, T. Moa ${ }^{45 a, 45 b}$, K. Mochizuki ${ }^{109}$, P. Mogg ${ }^{52}$, S. Mohapatra ${ }^{39}$, R. Moles-Valls ${ }^{24}$, M.C. Mondragon ${ }^{106}$, K. Mönig ${ }^{46}$, J. Monk ${ }^{40}$, E. Monnier ${ }^{101}$, A. Montalbano ${ }^{152}$, J. Montejo Berlingen $^{36}$, M. Montella ${ }^{94}$, F. Monticelli ${ }^{88}$, S. Monzani $^{68 a}$, N. Morange ${ }^{132}$, D. Moreno ${ }^{22}$, M. Moreno Llácer ${ }^{36}$, P. Morettini ${ }^{55 b}$, M. Morgenstern ${ }^{120}$, S. Morgenstern ${ }^{48}$, D. Mori ${ }^{152}$, M. Morii ${ }^{59}$, M. Morinaga ${ }^{179}$, V. Morisbak ${ }^{134}$, A.K. Morley ${ }^{36}$, G. Mornacchi ${ }^{36}$, A.P. Morris ${ }^{94}$, L. Morvaj ${ }^{155}$, P. Moschovakos ${ }^{10}$, B. Moser ${ }^{120}$, M. Mosidze ${ }^{159 b}$, H.J. Moss ${ }^{149}$, J. Moss ${ }^{31, \mathrm{~m}}$, K. Motohashi ${ }^{165}$, E. Mountricha ${ }^{36}$, E.J.W. Moyse ${ }^{102}$, S. Muanza ${ }^{101}$, F. Mueller ${ }^{115}$, J. Mueller ${ }^{139}$, R.S.P. Mueller ${ }^{114}$, D. Muenstermann ${ }^{89}$, G.A. Mullier ${ }^{96}$, J.L. Munoz Martinez ${ }^{14}$, F.J. Munoz Sanchez ${ }^{100}$, P. Murin ${ }^{28 b}$, W.J. Murray ${ }^{178,144}$, A. Murrone ${ }^{68 \mathrm{a}, 68 \mathrm{~b}}$, M. Muškinja ${ }^{18}$, C. Mwewa ${ }^{33 a}$, A.G. Myagkov ${ }^{123, \text { an }}$, J. Myers ${ }^{131}$, M. Myska ${ }^{142}$, B.P. Nachman ${ }^{18}$, O. Nackenhorst ${ }^{47}$, A.Nag Nag ${ }^{48}$, K. Nagai ${ }^{135}$, K. Nagano ${ }^{81}$, Y. Nagasaka ${ }^{62}$, M. Nagel $^{52}$, E. Nagy ${ }^{101}$, A.M. Nairz ${ }^{36}$, Y. Nakahama ${ }^{117}$, K. Nakamura ${ }^{81}$, T. Nakamura ${ }^{163}$, I. Nakano ${ }^{127}$, H. Nanjo ${ }^{133}$, F. Napolitano ${ }^{61 a}$, R.F. Naranjo Garcia ${ }^{46}$, R. Narayan ${ }^{11}$, D.I. Narrias Villar ${ }^{61 a}$, I. Naryshkin ${ }^{138}$, T. Naumann ${ }^{46}$, G. Navarro ${ }^{22}$, H.A. Neal ${ }^{105, *}$, P.Y. Nechaeva ${ }^{110}$, F. Nechansky ${ }^{46}$, T.J. Neep ${ }^{21}$, A. Negri ${ }^{70 a, 70 b}$, M. Negrini ${ }^{23 b}$, S. Nektarijevic ${ }^{119}$, C. Nellist ${ }^{53}$, M.E. Nelson ${ }^{135}$, S. Nemecek ${ }^{141}$, P. Nemethy ${ }^{124}$, M. Nessi ${ }^{36, d}$, M.S. Neubauer ${ }^{173}$, M. Neumann ${ }^{182}$, P.R. Newman ${ }^{21}$, T.Y. $\mathrm{Ng}^{63 c}$, Y.S. $\mathrm{Ng}^{19}$, Y.W.Y. $\mathrm{Ng}^{171}$, H.D.N. Nguyen ${ }^{101}$, T. Nguyen Manh ${ }^{109}$, E. Nibigira ${ }^{38}$, R.B. Nickerson ${ }^{135}$, R. Nicolaidou ${ }^{145}$, D.S. Nielsen ${ }^{40}$, J. Nielsen ${ }^{146}$, N. Nikiforou ${ }^{11}$, V. Nikolaenko ${ }^{123, \text { an }}$, I. Nikolic-Audit ${ }^{136}$, K. Nikolopoulos ${ }^{21}$, P. Nilsson ${ }^{29}$, H.R. Nindhito ${ }^{54}$,

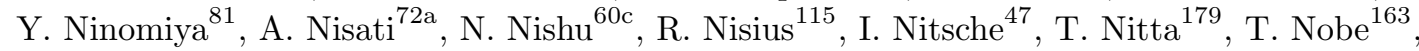
Y. Noguchi ${ }^{85}$, M. Nomachi ${ }^{133}$, I. Nomidis ${ }^{136}$, M.A. Nomura ${ }^{29}$, M. Nordberg ${ }^{36}$,

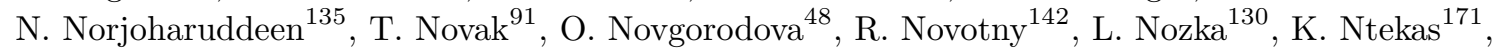
E. Nurse ${ }^{94}$, F. Nuti ${ }^{104}$, F.G. Oakham ${ }^{34, \text { av }}$, H. Oberlack ${ }^{115}$, J. Ocariz ${ }^{136}$, A. Ochi ${ }^{82}$, I. Ochoa ${ }^{39}$, J.P. Ochoa-Ricoux ${ }^{147 a}$, K. O'Connor ${ }^{26}$, S. Oda ${ }^{87}$, S. Odaka ${ }^{81}$, S. Oerdek ${ }^{53}$, A. Ogrodnik ${ }^{83 a}$, A. $\mathrm{Oh}^{100}$, S.H. $\mathrm{Oh}^{49}$, C.C. $\mathrm{Ohm}^{154}$, H. Oide ${ }^{55 b, 55 a}$, M.L. Ojeda ${ }^{167}$, H. Okawa ${ }^{169}$, Y. Okazaki ${ }^{85}$, Y. Okumura ${ }^{163}$, T. Okuyama ${ }^{81}$, A. Olariu ${ }^{27 b}$, L.F. Oleiro Seabra ${ }^{140 a}$, S.A. Olivares Pino ${ }^{147 a}$, 
D. Oliveira Damazio ${ }^{29}$, J.L. Oliver ${ }^{1}$, M.J.R. Olsson ${ }^{171}$, A. Olszewski ${ }^{84}$, J. Olszowska ${ }^{84}$, D.C. O'Neil ${ }^{152}$, A. Onofre ${ }^{140 a, 140 e}$, K. Onogi ${ }^{117}$, P.U.E. Onyisi ${ }^{11}$, H. Oppen ${ }^{134}$, M.J. Oreglia ${ }^{37}$, G.E. Orellana ${ }^{88}$, Y. Oren ${ }^{161}$, D. Orestano ${ }^{74 a, 74 b}$, N. Orlando ${ }^{14}$, R.S. Orr ${ }^{167}$, B. Osculati ${ }^{55 b, 55 a, *}$, V. O’Shea ${ }^{57}$, R. Ospanov ${ }^{60 a}$, G. Otero y Garzon ${ }^{30}$, H. Otono $^{87}$, M. Ouchrif ${ }^{35 d}$, F. Ould-Saada ${ }^{134}$, A. Ouraou ${ }^{145}$, Q. Ouyang ${ }^{15 a}$, M. Owen ${ }^{57}$, R.E. Owen ${ }^{21}$, V.E. Ozcan ${ }^{12 c}$, N. Ozturk ${ }^{8}$, J. Pacalt ${ }^{130}$, H.A. Pacey ${ }^{32}$, K. Pachal ${ }^{49}$, A. Pacheco Pages ${ }^{14}$, C. Padilla Aranda ${ }^{14}$, S. Pagan Griso ${ }^{18}$, M. Paganini ${ }^{183}$, G. Palacino ${ }^{65}$, S. Palazzo ${ }^{50}$, S. Palestini ${ }^{36}$, M. Palka ${ }^{83 b}$, D. Pallin ${ }^{38}$, I. Panagoulias ${ }^{10}$, C.E. Pandini ${ }^{36}$, J.G. Panduro Vazquez ${ }^{93}$, P. Pani ${ }^{46}$, G. Panizzo ${ }^{66 a, 66 c}$, L. Paolozzi ${ }^{54}$, C. Papadatos ${ }^{109}$, K. Papageorgiou ${ }^{9, h}$, A. Paramonov ${ }^{6}$, D. Paredes Hernandez ${ }^{63 b}$, S.R. Paredes Saenz ${ }^{135}$, B. Parida ${ }^{166}$, T.H. Park ${ }^{167}$, A.J. Parker ${ }^{89}$, M.A. Parker ${ }^{32}$, F. Parodi ${ }^{55 b, 55 a}$, E.W.P. Parrish ${ }^{121}$, J.A. Parsons ${ }^{39}$, U. Parzefall ${ }^{52}$, L. Pascual Dominguez ${ }^{136}$, V.R. Pascuzzi ${ }^{167}$, J.M.P. Pasner ${ }^{146}$, E. Pasqualucci ${ }^{72 a}$, S. Passaggio ${ }^{55 b}$, F. Pastore ${ }^{93}$, P. Pasuwan ${ }^{45 a, 45 b}$, S. Pataraia ${ }^{99}$, J.R. Pater ${ }^{100}$, A. Pathak ${ }^{181}$, T. Pauly ${ }^{36}$, B. Pearson ${ }^{115}$, M. Pedersen ${ }^{134}$, L. Pedraza Diaz ${ }^{119}$, R. Pedro ${ }^{140 a, 140 b}$, S.V. Peleganchuk ${ }^{122 b, 122 a}$, O. Penc ${ }^{141}$, C. Peng ${ }^{15 a}$, H. Peng ${ }^{60 a}$, B.S. Peralva ${ }^{80 a}$, M.M. Perego ${ }^{132}$, A.P. Pereira Peixoto ${ }^{140 a, 140 e}$, D.V. Perepelitsa ${ }^{29}$, F. Peri ${ }^{19}$, L. Perini ${ }^{68 a, 68 b}$, H. Pernegger ${ }^{36}$, S. Perrella ${ }^{69 a, 69 b}$, V.D. Peshekhonov ${ }^{79, *}$, K. Peters ${ }^{46}$, R.F.Y. Peters ${ }^{100}$, B.A. Petersen ${ }^{36}$, T.C. Petersen ${ }^{40}$, E. Petit ${ }^{58}$, A. Petridis ${ }^{1}$, C. Petridou ${ }^{162}$, P. Petroff ${ }^{132}$, M. Petrov ${ }^{135}$, F. Petrucci ${ }^{74 a, 74 b}$, M. Pettee ${ }^{183}$, N.E. Pettersson ${ }^{102}$, K. Petukhova $^{143}$, A. Peyaud ${ }^{145}$, R. Pezoa ${ }^{147 \mathrm{~b}}$, T. Pham ${ }^{104}$, F.H. Phillips ${ }^{106}$, P.W. Phillips ${ }^{144}$, M.W. Phipps ${ }^{173}$, G. Piacquadio ${ }^{155}$, E. Pianori ${ }^{18}$, A. Picazio ${ }^{102}$, R.H. Pickles ${ }^{100}$, R. Piegaia ${ }^{30}$, D. Pietreanu ${ }^{27 b}$, J.E. Pilcher ${ }^{37}$, A.D. Pilkington ${ }^{100}$, M. Pinamonti ${ }^{73 a, 73 b}$, J.L. Pinfold ${ }^{3}$, M. Pitt ${ }^{180}$, L. Pizzimento ${ }^{73 a, 73 b}$, M.-A. Pleier ${ }^{29}$, V. Pleskot ${ }^{143}$, E. Plotnikova ${ }^{79}$, D. Pluth ${ }^{78}$, P. Podberezko ${ }^{122 \mathrm{~b}, 122 \mathrm{a}}$, R. Poettgen ${ }^{96}$, R. Poggi ${ }^{54}$, L. Poggioli $^{132}$, I. Pogrebnyak ${ }^{106}$, D. Pohl $^{24}$, I. Pokharel ${ }^{53}$, G. Polesello ${ }^{70 a}$, A. Poley ${ }^{18}$, A. Policicchio ${ }^{72 a, 72 b}$, R. Polifka ${ }^{36}$, A. Polini ${ }^{23 b}$, C.S. Pollard ${ }^{46}$, V. Polychronakos ${ }^{29}$, D. Ponomarenko ${ }^{112}$, L. Pontecorvo ${ }^{36}$, S. Popa ${ }^{27 a}$, G.A. Popeneciu ${ }^{27 d}$, D.M. Portillo Quintero ${ }^{136}$, S. Pospisil ${ }^{142}$, K. Potamianos ${ }^{46}$, I.N. Potrap ${ }^{79}$, C.J. Potter ${ }^{32}$, H. Potti ${ }^{11}$, T. Poulsen ${ }^{96}$, J. Poveda ${ }^{36}$, T.D. Powell ${ }^{149}$, G. Pownall ${ }^{46}$, M.E. Pozo Astigarraga ${ }^{36}$, P. Pralavorio ${ }^{101}$, S. Prell ${ }^{78}$, D. Price ${ }^{100}$, M. Primavera ${ }^{67 a}$, S. Prince ${ }^{103}$, M.L. Proffitt ${ }^{148}$, N. Proklova ${ }^{112}$, K. Prokofiev ${ }^{63 c}$, F. Prokoshin ${ }^{147 b}$, S. Protopopescu ${ }^{29}$, J. Proudfoot ${ }^{6}$, M. Przybycien ${ }^{83 a}$, A. Puri ${ }^{173}$, P. Puzo ${ }^{132}$, J. $\operatorname{Qian}^{105}$, Y. Qin ${ }^{100}$, A. Quadt ${ }^{53}$, M. Queitsch-Maitland ${ }^{46}$, A. Qureshi ${ }^{1}$, P. Rados ${ }^{104}$, F. Ragusa ${ }^{68 a, 68 b}$, G. Rahal ${ }^{97}$, J.A. Raine ${ }^{54}$, S. Rajagopalan ${ }^{29}$, A. Ramirez Morales ${ }^{92}$, K. Ran ${ }^{15 a, 15 d}$, T. Rashid ${ }^{132}$, S. Raspopov ${ }^{5}$, M.G. Ratti ${ }^{68 a, 68 b}$, D.M. Rauch ${ }^{46}$, F. Rauscher ${ }^{114}$, S. Rave ${ }^{99}$, B. Ravina ${ }^{149}$, I. Ravinovich ${ }^{180}$, J.H. Rawling ${ }^{100}$, M. Raymond ${ }^{36}$, A.L. Read ${ }^{134}$, N.P. Readioff ${ }^{58}$, M. Reale ${ }^{67 a, 67 b}$,

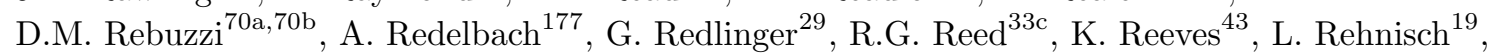
J. Reichert ${ }^{137}$, D. Reikher ${ }^{161}$, A. Reiss ${ }^{99}$, A. Rej ${ }^{151}$, C. Rembser ${ }^{36}$, H. Ren ${ }^{15 a}$, M. Rescigno ${ }^{72 a}$, S. Resconi ${ }^{68 \mathrm{a}}$, E.D. Resseguie ${ }^{137}$, S. Rettie ${ }^{175}$, E. Reynolds ${ }^{21}$, O.L. Rezanova ${ }^{122 \mathrm{~b}, 122 \mathrm{a}}$, P. Reznicek ${ }^{143}$, E. Ricci ${ }^{75 a, 75 b}$, R. Richter ${ }^{115}$, S. Richter ${ }^{46}$, E. Richter-Was ${ }^{83 b}$, O. Ricken ${ }^{24}$, M. Ridel ${ }^{136}$, P. Rieck $^{115}$, C.J. Riegel ${ }^{182}$, O. Rifki ${ }^{46}$, M. Rijssenbeek ${ }^{155}$, A. Rimoldi ${ }^{70 a, 70 b}$, M. Rimoldi ${ }^{20}$, L. Rinaldi ${ }^{23 b}$, G. Ripellino ${ }^{154}$, B. Ristic ${ }^{89}$, E. Ritsch ${ }^{36}$, I. Riu ${ }^{14}$, J.C. Rivera Vergara ${ }^{147 a}$, F. Rizatdinova ${ }^{129}$, E. Rizvi ${ }^{92}$, C. Rizzi ${ }^{36}$, R.T. Roberts ${ }^{100}$, S.H. Robertson ${ }^{103, \mathrm{ac}}$, M. Robin ${ }^{46}$, D. Robinson ${ }^{32}$, J.E.M. Robinson ${ }^{46}$, A. Robson ${ }^{57}$, E. Rocco ${ }^{99}$, C. Roda ${ }^{71 a, 71 b}$, Y. Rodina ${ }^{101}$, S. Rodriguez Bosca ${ }^{174}$, A. Rodriguez Perez ${ }^{14}$,

D. Rodriguez Rodriguez ${ }^{174}$, A.M. Rodríguez Vera ${ }^{168 b}, \mathrm{~S} . \mathrm{Roe}^{36}$, O. Røhne ${ }^{134}$, R. Röhrig ${ }^{115}$, C.P.A. Roland ${ }^{65}$, J. Roloff ${ }^{59}$, A. Romaniouk ${ }^{112}$, M. Romano ${ }^{23 b, 23 a}$, N. Rompotis ${ }^{90}$, M. Ronzani $^{124}$, L. Roos ${ }^{136}$, S. Rosati ${ }^{72 a}$, K. Rosbach ${ }^{52}$, N-A. $\operatorname{Rosien}^{53}$, G. Rosin ${ }^{102}$, B.J. Rosser ${ }^{137}$, E. Rossi ${ }^{46}$,

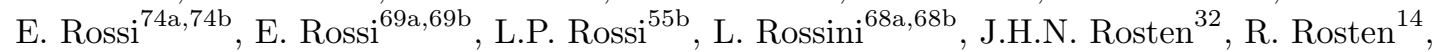
M. Rotaru ${ }^{27 b}$, J. Rothberg ${ }^{148}$, D. Rousseau ${ }^{132}$, D. Roy ${ }^{33 c}$, A. Rozanov ${ }^{101}$, Y. Rozen ${ }^{160}$, 
X. Ruan ${ }^{33 c}$, F. Rubbo ${ }^{153}$, F. Rühr ${ }^{52}$, A. Ruiz-Martinez ${ }^{174}$, A. Rummler ${ }^{36}$, Z. Rurikova ${ }^{52}$, N.A. Rusakovich ${ }^{79}$, H.L. Russell ${ }^{103}$, L. Rustige ${ }^{38,47}$, J.P. Rutherfoord ${ }^{7}$, E.M. Rüttinger ${ }^{46, j}$, Y.F. Ryabov ${ }^{138}$, M. Rybar ${ }^{39}$, G. Rybkin ${ }^{132}$, A. Ryzhov ${ }^{123}$, G.F. Rzehorz ${ }^{53}$, P. Sabatini ${ }^{53}$, G. Sabato ${ }^{120}$, S. Sacerdoti ${ }^{132}$, H.F-W. Sadrozinski ${ }^{146}$, R. Sadykov ${ }^{79}$, F. Safai Tehrani ${ }^{72 a}$, B. Safarzadeh Samani ${ }^{156}$, P. Saha ${ }^{121}$, S. Saha ${ }^{103}$, M. Sahinsoy ${ }^{61 a}$, A. Sahu ${ }^{182}$, M. Saimpert ${ }^{46}$, M. Saito ${ }^{163}$, T. Saito ${ }^{163}$, H. Sakamoto ${ }^{163}$, A. Sakharov ${ }^{124, a m}$, D. Salamani ${ }^{54}$, G. Salamanna ${ }^{74 a, 74 b}$, J.E. Salazar Loyola ${ }^{147 b}$, P.H. Sales De Bruin ${ }^{172}$, D. Salihagic ${ }^{15, *}$, A. Salnikov ${ }^{153}$, J. Salt ${ }^{174}$, D. Salvatore ${ }^{41 \mathrm{~b}, 41 \mathrm{a}}$, F. Salvatore ${ }^{156}$, A. Salvucci ${ }^{63 \mathrm{a}, 63 \mathrm{~b}, 63 \mathrm{c}}$, A. Salzburger ${ }^{36}$, J. Samarati ${ }^{36}$,

D. Sammel ${ }^{52}$, D. Sampsonidis ${ }^{162}$, D. Sampsonidou ${ }^{162}$, J. Sánchez ${ }^{174}$, A. Sanchez Pineda ${ }^{66 a, 66 c}$, H. Sandaker ${ }^{134}$, C.O. Sander ${ }^{46}$, M. Sandhoff ${ }^{182}$, C. Sandoval ${ }^{22}$, D.P.C. Sankey ${ }^{144}$, M. Sannino ${ }^{55 b, 55 a}$, Y. Sano ${ }^{117}$, A. Sansoni ${ }^{51}$, C. Santoni ${ }^{38}$, H. Santos ${ }^{140 a, 140 b}$, S.N. Santpur ${ }^{18}$, A. Santra ${ }^{174}$, A. Sapronov ${ }^{79}$, J.G. Saraiva ${ }^{140 a, 140 d}$, O. Sasaki ${ }^{81}$, K. Sato ${ }^{169}$, E. Sauvan ${ }^{5}$, P. Savard ${ }^{167, a v}$, N. Savic ${ }^{115}$, R. Sawada ${ }^{163}$, C. Sawyer ${ }^{144}$, L. Sawyer ${ }^{95, a k}$, C. Sbarra ${ }^{23 b}$, A. Sbrizzi ${ }^{23 a}$, T. Scanlon ${ }^{94}$, J. Schaarschmidt ${ }^{148}$, P. Schacht ${ }^{115}$, B.M. Schachtner ${ }^{114}$, D. Schaefer ${ }^{37}$, L. Schaefer ${ }^{137}$, J. Schaeffer ${ }^{99}$, S. Schaepe ${ }^{36}$, U. Schäfer ${ }^{99}$, A.C. Schaffer ${ }^{132}$, D. Schaile ${ }^{114}$, R.D. Schamberger ${ }^{155}$, N. Scharmberg ${ }^{100}$, V.A. Schegelsky ${ }^{138}$, D. Scheirich ${ }^{143}$ F. Schenck ${ }^{19}$, M. Schernau ${ }^{171}$, C. Schiavi ${ }^{55 b, 55 a}$, S. Schier ${ }^{146}$, L.K. Schildgen ${ }^{24}$, Z.M. Schillaci ${ }^{26}$, E.J. Schioppa ${ }^{36}$, M. Schioppa ${ }^{41 \mathrm{~b}, 41 \mathrm{a}}$, K.E. Schleicher ${ }^{52}$, S. Schlenker ${ }^{36}$,

K.R. Schmidt-Sommerfeld ${ }^{115}$, K. Schmieden ${ }^{36}$, C. Schmitt ${ }^{99}$, S. Schmitt ${ }^{46}$, S. Schmitz ${ }^{99}$, J.C. Schmoeckel ${ }^{46}$, U. Schnoor ${ }^{52}$, L. Schoeffel ${ }^{145}$, A. Schoening ${ }^{61 b}$, E. Schopf ${ }^{135}$, M. Schott ${ }^{99}$, J.F.P. Schouwenberg ${ }^{119}$, J. Schovancova ${ }^{36}$, S. Schramm ${ }^{54}$, F. Schroeder ${ }^{182}$, A. Schulte ${ }^{99}$, H-C. Schultz-Coulon ${ }^{61 a}$, M. Schumacher ${ }^{52}$, B.A. Schumm ${ }^{146}$, Ph. Schune ${ }^{145}$, A. Schwartzman ${ }^{153}$, T.A. Schwarz ${ }^{105}$, Ph. Schwemling ${ }^{145}$, R. Schwienhorst ${ }^{106}$, A. Sciandra ${ }^{24}$, G. Sciolla ${ }^{26}$, M. Scornajenghi ${ }^{41 b, 41 a}$, F. Scuri ${ }^{71 a}$, F. Scutti ${ }^{104}$, L.M. Scyboz ${ }^{115}$, C.D. Sebastiani ${ }^{72 a, 72 b}$ P. Seema ${ }^{19}$, S.C. Seidel ${ }^{118}$, A. Seiden ${ }^{146}$, T. Seiss ${ }^{37}$, J.M. Seixas ${ }^{80 b}$, G. Sekhniaidze ${ }^{69 a}$, K. Sekhon ${ }^{105}$, S.J. Sekula ${ }^{42}$, N. Semprini-Cesari ${ }^{23 \mathrm{~b}, 23 \mathrm{a}}$, S. $\mathrm{Sen}^{49}$, S. Senkin ${ }^{38}$, C. Serfon ${ }^{76}$, L. Serin ${ }^{132}$, L. Serkin ${ }^{66 a, 66 b}$, M. Sessa ${ }^{60 a}$, H. Severini ${ }^{128}$, F. Sforza ${ }^{170}$, A. Sfyrla ${ }^{54}$, E. Shabalina ${ }^{53}$, J.D. Shahinian ${ }^{146}$, N.W. Shaikh ${ }^{45 a, 45 b}$, D. Shaked Renous ${ }^{180}$, L.Y. Shan ${ }^{15 a}$, R. Shang ${ }^{173}$, J.T. Shank ${ }^{25}$, M. Shapiro ${ }^{18}$, A. Sharma ${ }^{135}$, A.S. Sharma ${ }^{1}$, P.B. Shatalov ${ }^{111}$, K. Shaw ${ }^{156}$, S.M. Shaw ${ }^{100}$, A. Shcherbakova ${ }^{138}$, Y. Shen ${ }^{128}$, N. Sherafati ${ }^{34}$, A.D. Sherman ${ }^{25}$, P. Sherwood ${ }^{94}$, L. Shi ${ }^{158, \text { ar }}$, S. Shimizu ${ }^{81}$, C.O. Shimmin ${ }^{183}$, Y. Shimogama ${ }^{179}$, M. Shimojima ${ }^{116}$, I.P.J. Shipsey ${ }^{135}$, S. Shirabe ${ }^{87}$, M. Shiyakova ${ }^{79, \text { aa }}$, J. Shlomi ${ }^{180}$, A. Shmeleva ${ }^{110}$, M.J. Shochet ${ }^{37}$, S. Shojaii ${ }^{104}$, D.R. Shope ${ }^{128}$, S. Shrestha ${ }^{126}$, E. Shulga ${ }^{180}$, P. Sicho ${ }^{141}$, A.M. Sickles ${ }^{173}$, P.E. Sidebo ${ }^{154}$, E. Sideras Haddad ${ }^{33 c}$, O. Sidiropoulou ${ }^{36}$, A. Sidotid $^{23 b, 23 a}$, F. Siegert ${ }^{48}$, Dj. Sijacki ${ }^{16}$, M. Silva Jr. ${ }^{181}$, M.V. Silva Oliveira ${ }^{80 a}$, S.B. Silverstein ${ }^{45 a}$, S. Simion ${ }^{132}$, E. Simioni ${ }^{99}$, M. Simon ${ }^{99}$, R. Simoniello ${ }^{99}$, P. Sinervo ${ }^{167}$, N.B. Sinev ${ }^{131}$, M. Sioli $^{23 b, 23 a}$, I. Siral ${ }^{105}$, S.Yu. Sivoklokov ${ }^{113}$, J. Sjölin ${ }^{45 a, 45 b}$, E. Skorda ${ }^{96}$, P. Skubic ${ }^{128}$, M. Slawinska ${ }^{84}$, K. Sliwa ${ }^{170}$, R. Slovak ${ }^{143}$, V. Smakhtin ${ }^{180}$, B.H. Smart ${ }^{144}$, J. Smiesko ${ }^{28 a}$, N. Smirnov ${ }^{112}$, S.Yu. Smirnov ${ }^{112}$, Y. Smirnov ${ }^{112}$, L.N. Smirnova ${ }^{113, s}$, O. Smirnova ${ }^{96}$, J.W. Smith ${ }^{53}$, M. Smizanska ${ }^{89}$, K. Smolek ${ }^{142}$, A. Smykiewicz ${ }^{84}$, A.A. Snesarev ${ }^{110}$, H.L. Snoek ${ }^{120}$, I.M. Snyder ${ }^{131}$, S. Snyder ${ }^{29}$, R. Sobie ${ }^{176, \text { ac }}$, A.M. Soffa ${ }^{171}$, A. Soffer ${ }^{161}$, A. Søgaard ${ }^{50}$, F. Sohns ${ }^{53}$, G. Sokhrannyi ${ }^{91}$, C.A. Solans Sanchez ${ }^{36}$, E.Yu. Soldatov ${ }^{112}$, U. Soldevila ${ }^{174}$, A.A. Solodkov ${ }^{123}$, A. Soloshenko ${ }^{79}$, O.V. Solovyanov ${ }^{123}$, V. Solovyev ${ }^{138}$, P. Sommer ${ }^{149}$, H. Son ${ }^{170}$, W. Song ${ }^{144}$, W.Y. Song ${ }^{168 b}$, A. Sopczak ${ }^{142}$, F. Sopkova ${ }^{28 b}$, C.L. Sotiropoulou ${ }^{71 a, 71 b}$, S. Sottocornola ${ }^{70 a, 70 b}$, R. Soualah ${ }^{66 a, 66 c, g}$, A.M. Soukharev ${ }^{122 b, 122 a}$, D. South ${ }^{46}$, S. Spagnolo ${ }^{67 a, 67 b}$, M. Spalla ${ }^{115}$, M. Spangenberg ${ }^{178}$, F. Spanò ${ }^{93}$, D. Sperlich ${ }^{19}$, T.M. Spieker ${ }^{61 a}$, R. Spighi ${ }^{23 b}$, G. Spigo $^{36}$, L.A. Spiller ${ }^{104}$, M. Spina ${ }^{156}$, D.P. Spiteri ${ }^{57}$, M. Spousta ${ }^{143}$, A. Stabile ${ }^{68 a, 68 b}$, B.L. Stamas ${ }^{121}$, R. Stamen $^{61 a}$, M. Stamenkovic ${ }^{120}$, S. Stamm ${ }^{19}$, E. Stanecka ${ }^{84}$, R.W. Stanek ${ }^{6}$, B. Stanislaus ${ }^{135}$, 
M.M. Stanitzki ${ }^{46}$, M. Stankaityte ${ }^{135}$, B. Stapf ${ }^{120}$, E.A. Starchenko ${ }^{123}$, G.H. Stark ${ }^{146}$, J. Stark ${ }^{58}$, S.H Stark ${ }^{40}$, P. Staroba ${ }^{141}$, P. Starovoitov ${ }^{61 a}$, S. Stärz ${ }^{103}$, R. Staszewski ${ }^{84}$, G. Stavropoulos ${ }^{44}$, M. Stegler ${ }^{46}$, P. Steinberg ${ }^{29}$, B. Stelzer ${ }^{152}$, H.J. Stelzer ${ }^{36}$, O. Stelzer-Chilton ${ }^{168 a}$, H. Stenzel ${ }^{56}$, T.J. Stevenson ${ }^{156}$, G.A. Stewart ${ }^{36}$, M.C. Stockton ${ }^{36}$, G. Stoicea ${ }^{27 b}$, M. Stolarski ${ }^{140 a}$, P. Stolte ${ }^{53}$, S. Stonjek ${ }^{115}$, A. Straessner ${ }^{48}$, J. Strandberg ${ }^{154}$, S. Strandberg ${ }^{45 a, 45 b}$, M. Strauss ${ }^{128}$, P. Strizenec ${ }^{28 b}$, R. Ströhmer ${ }^{177}$, D.M. Strom ${ }^{131}$, R. Stroynowski ${ }^{42}$, A. Strubig $^{50}$, S.A. Stucci ${ }^{29}$, B. Stugu ${ }^{17}$, J. Stupak ${ }^{128}$, N.A. Styles ${ }^{46}$, D. Su ${ }^{153}$, S. Suchek ${ }^{61 a}$, Y. Sugaya ${ }^{133}$, V.V. Sulin ${ }^{110}$, M.J. Sullivan ${ }^{90}$, D.M.S. Sultan ${ }^{54}$, S. Sultansoy ${ }^{4 c}$, T. Sumida ${ }^{85}$, S. Sun ${ }^{105}$, X. Sun ${ }^{3}$, K. Suruliz ${ }^{156}$, C.J.E. Suster ${ }^{157}$, M.R. Sutton ${ }^{156}$, S. Suzuki ${ }^{81}$, M. Svatos ${ }^{141}$, M. Swiatlowski ${ }^{37}$, S.P. Swift ${ }^{2}$, A. Sydorenko ${ }^{99}$, I. Sykora ${ }^{28 a}$, M. Sykora ${ }^{143}$, T. Sykora ${ }^{143}$, D. Ta ${ }^{99}$, K. Tackmann ${ }^{46, y}$,

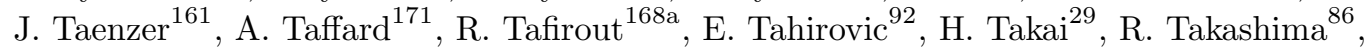
K. Takeda ${ }^{82}$, T. Takeshita ${ }^{150}$, E.P. Takeva ${ }^{50}$, Y. Takubo ${ }^{81}$, M. Talby ${ }^{101}$, A.A. Talyshev ${ }^{122 b, 122 a}$, N.M. Tamir ${ }^{161}$, J. Tanaka ${ }^{163}$, M. Tanaka ${ }^{165}$, R. Tanaka ${ }^{132}$, B.B. Tannenwald ${ }^{126}$,

S. Tapia Araya ${ }^{173}$, S. Tapprogge ${ }^{99}$, A. Tarek Abouelfadl Mohamed ${ }^{136}$, S. Tarem $^{160}$, G. Tarna $^{27 b, c}$, G.F. Tartarelli ${ }^{68 \mathrm{a}}$, P. Tas ${ }^{143}$, M. Tasevsky ${ }^{141}$, T. Tashiro ${ }^{85}$, E. Tassi ${ }^{41 \mathrm{~b}, 41 \mathrm{a}}$,

A. Tavares Delgado ${ }^{140 a, 140 b}$, Y. Tayalati ${ }^{35 e}$, A.J. Taylor ${ }^{50}$, G.N. Taylor ${ }^{104}$, P.T.E. Taylor ${ }^{104}$, W. Taylor ${ }^{168 b}$, A.S. Tee ${ }^{89}$, R. Teixeira De Lima ${ }^{153}$, P. Teixeira-Dias ${ }^{93}$, H. Ten Kate ${ }^{36}$, J.J. Teoh ${ }^{120}$, S. Terada ${ }^{81}$, K. Terashi ${ }^{163}$, J. Terron ${ }^{98}$, S. Terzo ${ }^{14}$, M. Testa ${ }^{51}$, R.J. Teuscher ${ }^{167, a c}$, S.J. Thais ${ }^{183}$, T. Theveneaux-Pelzer ${ }^{46}$, F. Thiele ${ }^{40}$, D.W. Thomas ${ }^{93}$, J.O. Thomas ${ }^{42}$, J.P. Thomas $^{21}$, A.S. Thompson ${ }^{57}$, P.D. Thompson ${ }^{21}$, L.A. Thomsen ${ }^{183}$, E. Thomson ${ }^{137}$, Y. $\operatorname{Tian}^{39}$, R.E. Ticse Torres ${ }^{53}$, V.O. Tikhomirov ${ }^{110, a o}$, Yu.A. Tikhonov ${ }^{122 b, 122 a}$,

S. Timoshenko ${ }^{112}$, P. Tipton ${ }^{183}$, S. Tisserant ${ }^{101}$, K. Todome ${ }^{23 \mathrm{~b}, 23 \mathrm{a}}$, S. Todorova-Nova ${ }^{5}$, S. Todt $^{48}$, J. Tojo ${ }^{87}$, S. Tokár ${ }^{28 a}$, K. Tokushuku ${ }^{81}$, E. Tolley ${ }^{126}$, K.G. Tomiwa ${ }^{33 c}$, M. Tomoto ${ }^{117}$,

L. Tompkins ${ }^{153, \mathrm{p}}, \mathrm{K}$. Toms $^{118}$, B. Tong ${ }^{59}$, P. Tornambe ${ }^{102}$, E. Torrence ${ }^{131}$, H. Torres ${ }^{48}$, E. Torró Pastor ${ }^{148}$, C. Tosciri ${ }^{135}$, J. Toth ${ }^{101, a b}$, D.R. Tovey ${ }^{149}$, C.J. Treado ${ }^{124}$, T. Trefzger ${ }^{177}$,

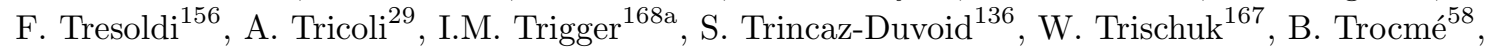

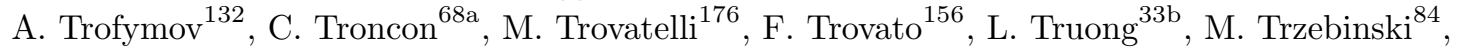

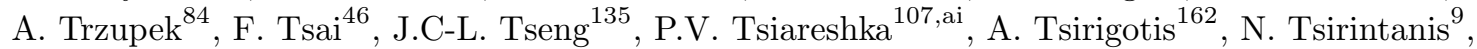
V. Tsiskaridze ${ }^{155}$, E.G. Tskhadadze ${ }^{159 a}$, M. Tsopoulou ${ }^{162}$, I.I. Tsukerman ${ }^{111}$, V. Tsulaia ${ }^{18}$,

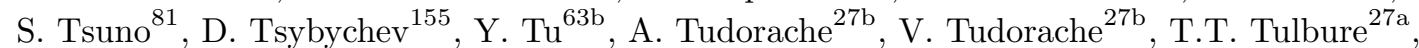
A.N. Tuna ${ }^{59}$, S. Turchikhin ${ }^{79}$, D. Turgeman ${ }^{180}$, I. Turk Cakir ${ }^{4 b, t}$, R.J. Turner ${ }^{21}$, R.T. Turra ${ }^{68 a}$,

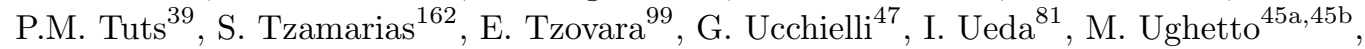

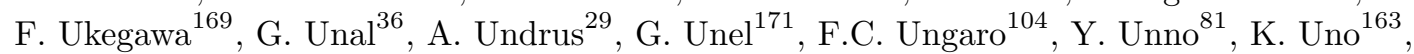

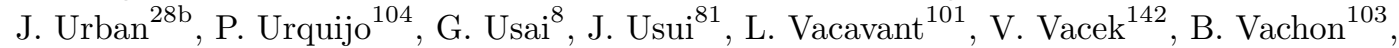
K.O.H. Vadla ${ }^{134}$, A. Vaidya ${ }^{94}$, C. Valderanis ${ }^{114}$, E. Valdes Santurio ${ }^{45 a, 45 b}$, M. Valente ${ }^{54}$, S. Valentinetti ${ }^{23 b, 23 a}$, A. Valero ${ }^{174}$, L. Valéry ${ }^{46}$, R.A. Vallance ${ }^{21}$, A. Vallier $^{36}$, J.A. Valls Ferrer ${ }^{174}$, T.R. Van Daalen ${ }^{14}$, P. Van Gemmeren ${ }^{6}$, I. Van Vulpen ${ }^{120}$, M. Vanadia ${ }^{73 a, 73 b}$, W. Vandelli ${ }^{36}$,

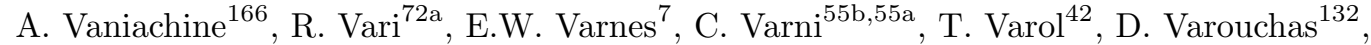
K.E. Varvell ${ }^{157}$, M.E. Vasile ${ }^{27 b}$, G.A. Vasquez ${ }^{176}$, J.G. Vasquez ${ }^{183}$, F. Vazeille ${ }^{38}$, D. Vazquez Furelos ${ }^{14}$, T. Vazquez Schroeder $^{36}$, J. Veatch ${ }^{53}$, V. Vecchio ${ }^{74 a, 74 b}$, L.M. Veloce ${ }^{167}$, F. Veloso ${ }^{140 a, 140 c}$, S. Veneziano ${ }^{72 a}$, A. Ventura ${ }^{67 a, 67 b}$, N. Venturi ${ }^{36}$, A. Verbytskyi ${ }^{115}$,

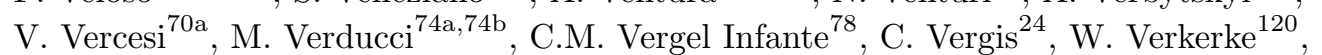
A.T. Vermeulen ${ }^{120}$, J.C. Vermeulen ${ }^{120}$, M.C. Vetterli ${ }^{152, a v}$, N. Viaux Maira ${ }^{147 b}$, M. Vicente Barreto Pinto ${ }^{54}$, I. Vichou ${ }^{173, *}$, T. Vickey ${ }^{149}$, O.E. Vickey Boeriu ${ }^{149}$, G.H.A. Viehhauser ${ }^{135}$, L. Vigani ${ }^{135}$, M. Villa ${ }^{23 b, 23 a}$, M. Villaplana Perez ${ }^{68 a, 68 b}$, E. Vilucchi ${ }^{51}$, M.G. Vincter ${ }^{34}$, V.B. Vinogradov ${ }^{79}$, A. Vishwakarma ${ }^{46}$, C. Vittori ${ }^{23 b, 23 a}$, I. Vivarelli ${ }^{156}$, M. Vogel ${ }^{182}$, P. Vokac $^{142}$, G. Volpi ${ }^{14}$, S.E. von Buddenbrock ${ }^{33 c}$, E. Von Toerne ${ }^{24}$, V. Vorobel ${ }^{143}$, K. Vorobev ${ }^{112}$, M. $\operatorname{Vos}^{174}$, J.H. Vossebeld ${ }^{90}$, N. Vranjes ${ }^{16}$, M. Vranjes Milosavljevic ${ }^{16}$, V. Vrba $^{142}$, 
M. Vreeswijk ${ }^{120}$, T. Šfiligoj ${ }^{91}$, R. Vuillermet ${ }^{36}$, I. Vukotic ${ }^{37}$, T. Ženiš ${ }^{28 a}$, L. Živković $^{16}$, P. Wagner ${ }^{24}$, W. Wagner ${ }^{182}$, J. Wagner-Kuhr ${ }^{114}, \mathrm{H}$. Wahlberg ${ }^{88}$, S. Wahrmund ${ }^{48}$, K. Wakamiya ${ }^{82}$, V.M. Walbrecht ${ }^{115}$, J. Walder ${ }^{89}$, R. Walker ${ }^{114}$, S.D. Walker ${ }^{93}$, W. Walkowiak ${ }^{151}$ V. Wallangen ${ }^{45 a, 45 b}$, A.M. Wang ${ }^{59}$, C. Wang ${ }^{60 b}$, F. Wang ${ }^{181}$, H. Wang ${ }^{18}$, H. Wang ${ }^{3}$, J. Wang ${ }^{157}$,

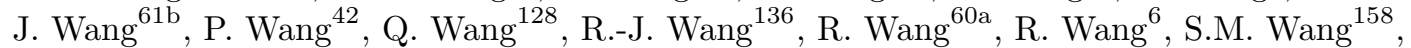

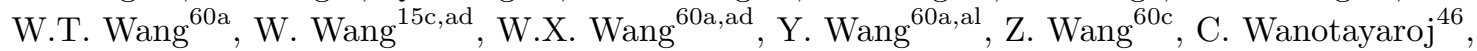
A. Warburton ${ }^{103}$, C.P. Ward ${ }^{32}$, D.R. Wardrope ${ }^{94}$, A. Washbrook ${ }^{50}$, A.T. Watson ${ }^{21}$, M.F. Watson ${ }^{21}$, G. Watts ${ }^{148}$, B.M. Waugh ${ }^{94}$, A.F. Webb ${ }^{11}$, S. Webb ${ }^{99}$, C. Weber ${ }^{183}$, M.S. Weber ${ }^{20}$, S.A. Weber ${ }^{34}$, S.M. Weber ${ }^{61 a}$, A.R. Weidberg ${ }^{135}$, J. Weingarten ${ }^{47}$, M. Weirich ${ }^{99}$, C. Weiser ${ }^{52}$, P.S. Wells ${ }^{36}$, T. Wenaus ${ }^{29}$, T. Wengler ${ }^{36}$, S. Wenig ${ }^{36}$, N. Wermes ${ }^{24}$, M.D. Werner ${ }^{78}$, P. Werner ${ }^{36}$, M. Wessels ${ }^{61 a}$, T.D. Weston ${ }^{20}$, K. Whalen ${ }^{131}$, N.L. Whallon ${ }^{148}$, A.M. Wharton ${ }^{89}$, A.S. White ${ }^{105}$, A. White ${ }^{8}$, M.J. White ${ }^{1}$, R. White ${ }^{147 b}$, D. Whiteson ${ }^{171}$, B.W. Whitmore ${ }^{89}$, F.J. Wickens ${ }^{144}$, W. Wiedenmann ${ }^{181}$, M. Wielers ${ }^{144}$, C. Wiglesworth ${ }^{40}$, L.A.M. Wiik-Fuchs ${ }^{52}$, F. Wilk ${ }^{100}$, H.G. Wilkens ${ }^{36}$, L.J. Wilkins ${ }^{93}$, H.H. Williams ${ }^{137}$, S. Williams ${ }^{32}$, C. Willis ${ }^{106}$, S. Willocq ${ }^{102}$, J.A. Wilson ${ }^{21}$, I. Wingerter-Seez ${ }^{5}$, E. Winkels ${ }^{156}$, F. Winklmeier ${ }^{131}$, O.J. Winston ${ }^{156}$, B.T. Winter ${ }^{52}$, M. Wittgen ${ }^{153}$, M. Wobisch ${ }^{95}$, A. Wolf ${ }^{99}$, T.M.H. Wolf ${ }^{120}$ R. Wolff ${ }^{101}$, R.W. Wölker ${ }^{135}$, J. Wollrath ${ }^{52}$, M.W. Wolter ${ }^{84}$, H. Wolters ${ }^{140 a, 140 c}$, V.W.S. Wong ${ }^{175}$, N.L. Woods ${ }^{146}$, S.D. Worm ${ }^{21}$, B.K. Wosiek ${ }^{84}$, K.W. Woźniak ${ }^{84}$, K. Wraight ${ }^{57}$, S.L. Wu ${ }^{181}$, X. $\mathrm{Wu}^{54}$, Y. Wu ${ }^{60 a}$, T.R. Wyatt ${ }^{100}$, B.M. Wynne ${ }^{50}$, S. Xella ${ }^{40}$, Z. Xi ${ }^{105}$, L. Xia ${ }^{178}$, D. Xu ${ }^{15 a}$, H. Xu ${ }^{60 a, c}$, L. $\mathrm{Xu}^{29}$, T. $\mathrm{Xu}^{145}$, W. Xu ${ }^{105}$, Z. Xu ${ }^{60 \mathrm{~b}}$, Z. Xu ${ }^{153}$, B. Yabsley ${ }^{157}$, S. Yacoob ${ }^{33 a}$, K. Yajima ${ }^{133}$, D.P. Yallup ${ }^{94}$, D. Yamaguchi ${ }^{165}$, Y. Yamaguchi ${ }^{165}$, A. Yamamoto ${ }^{81}$, T. Yamanaka ${ }^{163}$, F. Yamane ${ }^{82}$, M. Yamatani ${ }^{163}$, T. Yamazaki ${ }^{163}$, Y. Yamazaki ${ }^{82}$, Z. Yan $^{25}$,

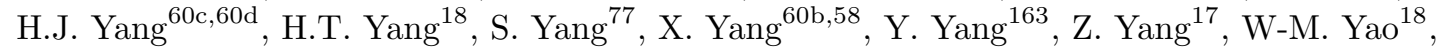
Y.C. Yap ${ }^{46}$, Y. Yasu ${ }^{81}$, E. Yatsenko ${ }^{60 c, 60 d}$, J. Ye ${ }^{42}$, S. Ye $^{29}$, I. Yeletskikh ${ }^{79}$, E. Yigitbasi ${ }^{25}$, E. Yildirim ${ }^{99}$, K. Yorita ${ }^{179}$, K. Yoshihara ${ }^{137}$, C.J.S. Young ${ }^{36}$, C. Young ${ }^{153}$, J. Yu $^{78}$, X. Yue ${ }^{61 a}$, S.P.Y. Yuen ${ }^{24}$, B. Zabinski ${ }^{84}$, G. Zacharis ${ }^{10}$, E. Zaffaroni ${ }^{54}$, J. Zahreddine ${ }^{136}$, R. Zaidan ${ }^{14}$, A.M. Zaitsev ${ }^{123, a n}$, T. Zakareishvili ${ }^{159 b}$, N. Zakharchuk ${ }^{34}$, S. Zambito ${ }^{59}$, D. Zanzi ${ }^{36}$, D.R. Zaripovas ${ }^{57}$, S.V. Zeißner ${ }^{47}$, C. Zeitnitz ${ }^{182}$, G. Zemaityte ${ }^{135}$, J.C. Zeng ${ }^{173}$, O. Zenin ${ }^{123}$, D. Zerwas ${ }^{132}$, M. Zgubic ${ }^{135}$, D.F. Zhang ${ }^{15 b}$, F. Zhang ${ }^{181}$, G. Zhang ${ }^{60 a}$, G. Zhang ${ }^{15 b}$, H. Zhang ${ }^{15 c}$, J. Zhang ${ }^{6}$, L. Zhang ${ }^{15 c}$, L. Zhang ${ }^{60 a}$, M. Zhang ${ }^{173}$, R. Zhang ${ }^{60 a}$, R. Zhang ${ }^{24}$, X. Zhang ${ }^{60 b}$, Y. Zhang ${ }^{15 a, 15 d}$, Z. Zhang ${ }^{63 a}$, Z. Zhang ${ }^{132}$, P. Zhao ${ }^{49}$, Y. Zhao ${ }^{60 b}$, Z. Zhao ${ }^{60 a}$, A. Zhemchugov ${ }^{79}$, Z. Zheng ${ }^{105}$, D. Zhong ${ }^{173}$, B. Zhou ${ }^{105}$, C. Zhou ${ }^{181}$, M.S. Zhou ${ }^{15 a, 15 d}$, M. Zhou ${ }^{155}$, N. Zhou ${ }^{60 c}$, Y. Zhou ${ }^{7}$, C.G. Zhu ${ }^{60 b}$, H.L. Zhu ${ }^{60 a}$, H. Zhu ${ }^{15 a}$, J. Zhu ${ }^{105}$, Y. Zhu ${ }^{60 a}$, X. Zhuang ${ }^{15 a}$, K. Zhukov ${ }^{110}$, V. Zhulanov ${ }^{122 b, 122 a}$, D. Zieminska ${ }^{65}$, N.I. Zimine ${ }^{79}$, S. Zimmermann ${ }^{52}$, Z. Zinonos ${ }^{115}$, M. Ziolkowski ${ }^{151}$, G. Zobernig ${ }^{181}$, A. Zoccoli ${ }^{23 b, 23 a}$, K. Zoch ${ }^{53}$, T.G. Zorbas ${ }^{149}$, R. Zou ${ }^{37}$, L. Zwalinski ${ }^{36}$

1 Department of Physics, University of Adelaide, Adelaide, Australia

2 Physics Department, SUNY Albany, Albany NY, United States of America

3 Department of Physics, University of Alberta, Edmonton AB, Canada

4 (a) Department of Physics, Ankara University, Ankara; ${ }^{(b)}$ Istanbul Aydin University, Istanbul;

${ }^{(c)}$ Division of Physics, TOBB University of Economics and Technology, Ankara, Turkey

5 LAPP, Université Grenoble Alpes, Université Savoie Mont Blanc, CNRS/IN2P3, Annecy, France

6 High Energy Physics Division, Argonne National Laboratory, Argonne IL, United States of America

7 Department of Physics, University of Arizona, Tucson AZ, United States of America

8 Department of Physics, University of Texas at Arlington, Arlington TX, United States of America

9 Physics Department, National and Kapodistrian University of Athens, Athens, Greece

10 Physics Department, National Technical University of Athens, Zografou, Greece

11 Department of Physics, University of Texas at Austin, Austin TX, United States of America 
$12{ }^{(a)}$ Bahcesehir University, Faculty of Engineering and Natural Sciences, Istanbul; ${ }^{(b)}$ Istanbul Bilgi University, Faculty of Engineering and Natural Sciences, Istanbul; ${ }^{(c)}$ Department of Physics, Bogazici University, Istanbul; ${ }^{(d)}$ Department of Physics Engineering, Gaziantep University, Gaziantep, Turkey

13 Institute of Physics, Azerbaijan Academy of Sciences, Baku, Azerbaijan

14 Institut de Física d'Altes Energies (IFAE), Barcelona Institute of Science and Technology, Barcelona, Spain

$15{ }^{(a)}$ Institute of High Energy Physics, Chinese Academy of Sciences, Beijing; ${ }^{(b)}$ Physics Department, Tsinghua University, Beijing; ${ }^{(c)}$ Department of Physics, Nanjing University, Nanjing; ${ }^{(d)}$ University of Chinese Academy of Science (UCAS), Beijing, China

16 Institute of Physics, University of Belgrade, Belgrade, Serbia

17 Department for Physics and Technology, University of Bergen, Bergen, Norway

18 Physics Division, Lawrence Berkeley National Laboratory and University of California, Berkeley CA, United States of America

19 Institut für Physik, Humboldt Universität zu Berlin, Berlin, Germany

20 Albert Einstein Center for Fundamental Physics and Laboratory for High Energy Physics, University of Bern, Bern, Switzerland

21 School of Physics and Astronomy, University of Birmingham, Birmingham, United Kingdom

22 Facultad de Ciencias y Centro de Investigaciónes, Universidad Antonio Nariño, Bogota, Colombia

$23{ }^{(a)}$ INFN Bologna and Universita' di Bologna, Dipartimento di Fisica; ${ }^{(b)}$ INFN Sezione di Bologna, Italy

24 Physikalisches Institut, Universität Bonn, Bonn, Germany

25 Department of Physics, Boston University, Boston MA, United States of America

26 Department of Physics, Brandeis University, Waltham MA, United States of America

27 (a) Transilvania University of Brasov, Brasov; ${ }^{(b)}$ Horia Hulubei National Institute of Physics and Nuclear Engineering, Bucharest; ${ }^{(c)}$ Department of Physics, Alexandru Ioan Cuza University of Iasi, Iasi; ${ }^{(d)}$ National Institute for Research and Development of Isotopic and Molecular Technologies, Physics Department, Cluj-Napoca; ${ }^{\left({ }^{e}\right)}$ University Politehnica Bucharest, Bucharest; ${ }^{(f)}$ West University in Timisoara, Timisoara, Romania

$28{ }^{(a)}$ Faculty of Mathematics, Physics and Informatics, Comenius University, Bratislava;

${ }^{(b)}$ Department of Subnuclear Physics, Institute of Experimental Physics of the Slovak Academy of Sciences, Kosice, Slovak Republic

29 Physics Department, Brookhaven National Laboratory, Upton NY, United States of America

30 Departamento de Física, Universidad de Buenos Aires, Buenos Aires, Argentina

31 California State University, CA, United States of America

32 Cavendish Laboratory, University of Cambridge, Cambridge, United Kingdom

$33{ }^{(a)}$ Department of Physics, University of Cape Town, Cape Town; ${ }^{(b)}$ Department of Mechanical Engineering Science, University of Johannesburg, Johannesburg; ${ }^{(c)}$ School of Physics, University of the Witwatersrand, Johannesburg, South Africa

34 Department of Physics, Carleton University, Ottawa ON, Canada

35 (a) Faculté des Sciences Ain Chock, Réseau Universitaire de Physique des Hautes Energies Université Hassan II, Casablanca; ${ }^{(b)}$ Faculté des Sciences, Université Ibn-Tofail, Kénitra;

${ }^{(c)}$ Faculté des Sciences Semlalia, Université Cadi Ayyad, LPHEA-Marrakech; ${ }^{(d)}$ Faculté des Sciences, Université Mohamed Premier and LPTPM, Oujda; ${ }^{(e)}$ Faculté des sciences, Université Mohammed V, Rabat, Morocco

36 CERN, Geneva, Switzerland

37 Enrico Fermi Institute, University of Chicago, Chicago IL, United States of America

38 LPC, Université Clermont Auvergne, CNRS/IN2P3, Clermont-Ferrand, France

39 Nevis Laboratory, Columbia University, Irvington NY, United States of America

40 Niels Bohr Institute, University of Copenhagen, Copenhagen, Denmark

$41{ }^{(a)}$ Dipartimento di Fisica, Università della Calabria, Rende; ${ }^{(b)}$ INFN Gruppo Collegato di Cosenza, Laboratori Nazionali di Frascati, Italy 


\section{of America}

60 (a) Department of Modern Physics and State Key Laboratory of Particle Detection and Electronics, University of Science and Technology of China, Hefei; ${ }^{(b)}$ Institute of Frontier and Interdisciplinary Science and Key Laboratory of Particle Physics and Particle Irradiation (MOE), Shandong University, Qingdao; ${ }^{(c)}$ School of Physics and Astronomy, Shanghai Jiao Tong University, KLPPAC-MoE, SKLPPC, Shanghai; ${ }^{(d)}$ Tsung-Dao Lee Institute, Shanghai, China

$61{ }^{(a)}$ Kirchhoff-Institut für Physik, Ruprecht-Karls-Universität Heidelberg, Heidelberg;

${ }^{(b)}$ Physikalisches Institut, Ruprecht-Karls-Universität Heidelberg, Heidelberg, Germany

62 Faculty of Applied Information Science, Hiroshima Institute of Technology, Hiroshima, Japan

$63{ }^{(a)}$ Department of Physics, Chinese University of Hong Kong, Shatin, N.T., Hong Kong;

${ }^{(b)}$ Department of Physics, University of Hong Kong, Hong Kong; ${ }^{(c)}$ Department of Physics and Institute for Advanced Study, Hong Kong University of Science and Technology, Clear Water Bay, Kowloon, Hong Kong, China

64 Department of Physics, National Tsing Hua University, Hsinchu, Taiwan

65 Department of Physics, Indiana University, Bloomington IN, United States of America

$66{ }^{(a)}$ INFN Gruppo Collegato di Udine, Sezione di Trieste, Udine; ${ }^{(b)}$ ICTP, Trieste; ${ }^{(c)}$ Dipartimento Politecnico di Ingegneria e Architettura, Università di Udine, Udine, Italy

$67{ }^{(a)}$ INFN Sezione di Lecce; ${ }^{(b)}$ Dipartimento di Matematica e Fisica, Università del Salento, Lecce, Italy

68 (a) INFN Sezione di Milano; ${ }^{(b)}$ Dipartimento di Fisica, Università di Milano, Milano, Italy

69 (a) INFN Sezione di Napoli; ${ }^{(b)}$ Dipartimento di Fisica, Università di Napoli, Napoli, Italy

$70 \quad{ }^{(a)}$ INFN Sezione di Pavia; ${ }^{(b)}$ Dipartimento di Fisica, Università di Pavia, Pavia, Italy

71 (a) INFN Sezione di Pisa; ${ }^{(b)}$ Dipartimento di Fisica E. Fermi, Università di Pisa, Pisa, Italy

72 (a) INFN Sezione di Roma; ${ }^{(b)}$ Dipartimento di Fisica, Sapienza Università di Roma, Roma, Italy

73 (a) INFN Sezione di Roma Tor Vergata; ${ }^{(b)}$ Dipartimento di Fisica, Università di Roma Tor Vergata, Roma, Italy

74 (a) INFN Sezione di Roma Tre; ${ }^{(b)}$ Dipartimento di Matematica e Fisica, Università Roma Tre, Roma, Italy

75 (a) INFN-TIFPA; ${ }^{(b)}$ Università degli Studi di Trento, Trento, Italy

76 Institut für Astro und Teilchenphysik, Leopold-Franzens-Universität, Innsbruck, Austria

77 University of Iowa, Iowa City IA, United States of America

78 Department of Physics and Astronomy, Iowa State University, Ames IA, United States of America

79 Joint Institute for Nuclear Research, Dubna, Russia 
${ }^{(a)}$ Departamento de Engenharia Elétrica, Universidade Federal de Juiz de Fora (UFJF), Juiz de Fora; ${ }^{(b)}$ Universidade Federal do Rio De Janeiro COPPE/EE/IF, Rio de Janeiro; ${ }^{(c)}$ Universidade Federal de São João del Rei (UFSJ), São João del Rei; ${ }^{\left({ }^{()}\right)}$Instituto de Física, Universidade de São Paulo, São Paulo, Brazil

81 KEK, High Energy Accelerator Research Organization, Tsukuba, Japan

82 Graduate School of Science, Kobe University, Kobe, Japan

83 (a) AGH University of Science and Technology, Faculty of Physics and Applied Computer Science, Krakow; ${ }^{(b)}$ Marian Smoluchowski Institute of Physics, Jagiellonian University, Krakow, Poland

84 Institute of Nuclear Physics Polish Academy of Sciences, Krakow, Poland

85 Faculty of Science, Kyoto University, Kyoto, Japan

86 Kyoto University of Education, Kyoto, Japan

87 Research Center for Advanced Particle Physics and Department of Physics, Kyushu University, Fukuoka, Japan

88 Instituto de Física La Plata, Universidad Nacional de La Plata and CONICET, La Plata, Argentina

89 Physics Department, Lancaster University, Lancaster, United Kingdom

90 Oliver Lodge Laboratory, University of Liverpool, Liverpool, United Kingdom

91 Department of Experimental Particle Physics, Jožef Stefan Institute and Department of Physics, University of Ljubljana, Ljubljana, Slovenia

92 School of Physics and Astronomy, Queen Mary University of London, London, United Kingdom

93 Department of Physics, Royal Holloway University of London, Egham, United Kingdom

94 Department of Physics and Astronomy, University College London, London, United Kingdom

95 Louisiana Tech University, Ruston LA, United States of America

96 Fysiska institutionen, Lunds universitet, Lund, Sweden

97 Centre de Calcul de l'Institut National de Physique Nucléaire et de Physique des Particules (IN2P3), Villeurbanne, France

98 Departamento de Física Teorica C-15 and CIAFF, Universidad Autónoma de Madrid, Madrid, Spain

99 Institut für Physik, Universität Mainz, Mainz, Germany

100 School of Physics and Astronomy, University of Manchester, Manchester, United Kingdom

101 CPPM, Aix-Marseille Université, CNRS/IN2P3, Marseille, France

102 Department of Physics, University of Massachusetts, Amherst MA, United States of America

103 Department of Physics, McGill University, Montreal QC, Canada

104 School of Physics, University of Melbourne, Victoria, Australia

105 Department of Physics, University of Michigan, Ann Arbor MI, United States of America

106 Department of Physics and Astronomy, Michigan State University, East Lansing MI, United States of America

107 B.I. Stepanov Institute of Physics, National Academy of Sciences of Belarus, Minsk, Belarus

108 Research Institute for Nuclear Problems of Byelorussian State University, Minsk, Belarus

109 Group of Particle Physics, University of Montreal, Montreal QC, Canada

110 P.N. Lebedev Physical Institute of the Russian Academy of Sciences, Moscow, Russia

111 Institute for Theoretical and Experimental Physics of the National Research Centre Kurchatov Institute, Moscow, Russia

112 National Research Nuclear University MEPhI, Moscow, Russia

113 D.V. Skobeltsyn Institute of Nuclear Physics, M.V. Lomonosov Moscow State University, Moscow, Russia

114 Fakultät für Physik, Ludwig-Maximilians-Universität München, München, Germany

115 Max-Planck-Institut für Physik (Werner-Heisenberg-Institut), München, Germany

116 Nagasaki Institute of Applied Science, Nagasaki, Japan

117 Graduate School of Science and Kobayashi-Maskawa Institute, Nagoya University, Nagoya, Japan

118 Department of Physics and Astronomy, University of New Mexico, Albuquerque NM, United States of America

119 Institute for Mathematics, Astrophysics and Particle Physics, Radboud University Nijmegen/Nikhef, Nijmegen, Netherlands 
Nikhef National Institute for Subatomic Physics and University of Amsterdam, Amsterdam, Netherlands

121 Department of Physics, Northern Illinois University, DeKalb IL, United States of America

$122{ }^{(a)}$ Budker Institute of Nuclear Physics and NSU, SB RAS, Novosibirsk; ${ }^{\left({ }^{b}\right)}$ Novosibirsk State University Novosibirsk, Russia

${ }^{123}$ Institute for High Energy Physics of the National Research Centre Kurchatov Institute, Protvino, Russia

124 Department of Physics, New York University, New York NY, United States of America

125 Ochanomizu University, Otsuka, Bunkyo-ku, Tokyo, Japan

126 Ohio State University, Columbus OH, United States of America

127 Faculty of Science, Okayama University, Okayama, Japan

128 Homer L. Dodge Department of Physics and Astronomy, University of Oklahoma, Norman OK, United States of America

129 Department of Physics, Oklahoma State University, Stillwater OK, United States of America

130 Palacký University, RCPTM, Joint Laboratory of Optics, Olomouc, Czech Republic

131 Center for High Energy Physics, University of Oregon, Eugene OR, United States of America

${ }^{132}$ LAL, Université Paris-Sud, CNRS/IN2P3, Université Paris-Saclay, Orsay, France

133 Graduate School of Science, Osaka University, Osaka, Japan

134 Department of Physics, University of Oslo, Oslo, Norway

135 Department of Physics, Oxford University, Oxford, United Kingdom

${ }^{136}$ LPNHE, Sorbonne Université, Paris Diderot Sorbonne Paris Cité, CNRS/IN2P3, Paris, France

137 Department of Physics, University of Pennsylvania, Philadelphia PA, United States of America

138 Konstantinov Nuclear Physics Institute of National Research Centre "Kurchatov Institute", PNPI, St. Petersburg, Russia

139 Department of Physics and Astronomy, University of Pittsburgh, Pittsburgh PA, United States of America

$140{ }^{(a)}$ Laboratório de Instrumentação e Física Experimental de Partículas - LIP; ${ }^{(b)}$ Departamento de Física, Faculdade de Ciências, Universidade de Lisboa, Lisboa; ${ }^{(c)}$ Departamento de Física, Universidade de Coimbra, Coimbra; ${ }^{(d)}$ Centro de Física Nuclear da Universidade de Lisboa, Lisboa; ${ }^{(e)}$ Departamento de Física, Universidade do Minho, Braga; ${ }^{(f)}$ Universidad de Granada, Granada (Spain); ${ }^{(g)}$ Dep Física and CEFITEC of Faculdade de Ciências e Tecnologia, Universidade Nova de Lisboa, Caparica, Portugal

141 Institute of Physics of the Czech Academy of Sciences, Prague, Czech Republic

${ }_{142}$ Czech Technical University in Prague, Prague, Czech Republic

143 Charles University, Faculty of Mathematics and Physics, Prague, Czech Republic

144 Particle Physics Department, Rutherford Appleton Laboratory, Didcot, United Kingdom

145 IRFU, CEA, Université Paris-Saclay, Gif-sur-Yvette, France

146 Santa Cruz Institute for Particle Physics, University of California Santa Cruz, Santa Cruz CA, United States of America

$147{ }^{(a)}$ Departamento de Física, Pontificia Universidad Católica de Chile, Santiago; ${ }^{(b)}$ Departamento de Física, Universidad Técnica Federico Santa María, Valparaíso, Chile

148 Department of Physics, University of Washington, Seattle WA, United States of America

149 Department of Physics and Astronomy, University of Sheffield, Sheffield, United Kingdom

150 Department of Physics, Shinshu University, Nagano, Japan

151 Department Physik, Universität Siegen, Siegen, Germany

152 Department of Physics, Simon Fraser University, Burnaby BC, Canada

153 SLAC National Accelerator Laboratory, Stanford CA, United States of America

154 Physics Department, Royal Institute of Technology, Stockholm, Sweden

155 Departments of Physics and Astronomy, Stony Brook University, Stony Brook NY, United States of America

156 Department of Physics and Astronomy, University of Sussex, Brighton, United Kingdom

157 School of Physics, University of Sydney, Sydney, Australia 
Institute of Physics, Academia Sinica, Taipei, Taiwan

$159{ }^{(a)}$ E. Andronikashvili Institute of Physics, Iv. Javakhishvili Tbilisi State University, Tbilisi; ${ }^{(b)}$ High Energy Physics Institute, Tbilisi State University, Tbilisi, Georgia

160 Department of Physics, Technion, Israel Institute of Technology, Haifa, Israel

161 Raymond and Beverly Sackler School of Physics and Astronomy, Tel Aviv University, Tel Aviv, Israel

162 Department of Physics, Aristotle University of Thessaloniki, Thessaloniki, Greece

163 International Center for Elementary Particle Physics and Department of Physics, University of Tokyo, Tokyo, Japan

164 Graduate School of Science and Technology, Tokyo Metropolitan University, Tokyo, Japan

165 Department of Physics, Tokyo Institute of Technology, Tokyo, Japan

166 Tomsk State University, Tomsk, Russia

167 Department of Physics, University of Toronto, Toronto ON, Canada

$168{ }^{(a)}$ TRIUMF, Vancouver BC; ${ }^{(b)}$ Department of Physics and Astronomy, York University, Toronto ON, Canada

169 Division of Physics and Tomonaga Center for the History of the Universe, Faculty of Pure and Applied Sciences, University of Tsukuba, Tsukuba, Japan

170 Department of Physics and Astronomy, Tufts University, Medford MA, United States of America

171 Department of Physics and Astronomy, University of California Irvine, Irvine CA, United States of America

172 Department of Physics and Astronomy, University of Uppsala, Uppsala, Sweden

173 Department of Physics, University of Illinois, Urbana IL, United States of America

174 Instituto de Física Corpuscular (IFIC), Centro Mixto Universidad de Valencia - CSIC, Valencia, Spain

175 Department of Physics, University of British Columbia, Vancouver BC, Canada

176 Department of Physics and Astronomy, University of Victoria, Victoria BC, Canada

177 Fakultät für Physik und Astronomie, Julius-Maximilians-Universität Würzburg, Würzburg, Germany

178 Department of Physics, University of Warwick, Coventry, United Kingdom

179 Waseda University, Tokyo, Japan

180 Department of Particle Physics, Weizmann Institute of Science, Rehovot, Israel

181 Department of Physics, University of Wisconsin, Madison WI, United States of America

182 Fakultät für Mathematik und Naturwissenschaften, Fachgruppe Physik, Bergische Universität Wuppertal, Wuppertal, Germany

183 Department of Physics, Yale University, New Haven CT, United States of America

184 Yerevan Physics Institute, Yerevan, Armenia

a Also at Centre for High Performance Computing, CSIR Campus, Rosebank, Cape Town, South Africa

${ }^{b}$ Also at CERN, Geneva, Switzerland

c Also at CPPM, Aix-Marseille Université, CNRS/IN2P3, Marseille, France

d Also at Département de Physique Nucléaire et Corpusculaire, Université de Genève, Genève, Switzerland

e Also at Departament de Fisica de la Universitat Autonoma de Barcelona, Barcelona, Spain

${ }^{f}$ Also at Departamento de Física, Instituto Superior Técnico, Universidade de Lisboa, Lisboa, Portugal

$g$ Also at Department of Applied Physics and Astronomy, University of Sharjah, Sharjah, United Arab Emirates

${ }^{h}$ Also at Department of Financial and Management Engineering, University of the Aegean, Chios, Greece

${ }^{i}$ Also at Department of Physics and Astronomy, University of Louisville, Louisville, KY, United States of America 
${ }^{j}$ Also at Department of Physics and Astronomy, University of Sheffield, Sheffield, United Kingdom ${ }^{k}$ Also at Department of Physics, California State University, East Bay, United States of America

${ }^{l}$ Also at Department of Physics, California State University, Fresno, United States of America

$m$ Also at Department of Physics, California State University, Sacramento, United States of America

$n$ Also at Department of Physics, King's College London, London, United Kingdom

- Also at Department of Physics, St. Petersburg State Polytechnical University, St. Petersburg, Russia

${ }^{p}$ Also at Department of Physics, Stanford University, Stanford CA, United States of America

$q$ Also at Department of Physics, University of Fribourg, Fribourg, Switzerland

${ }^{r}$ Also at Department of Physics, University of Michigan, Ann Arbor MI, United States of America

$s$ Also at Faculty of Physics, M.V. Lomonosov Moscow State University, Moscow, Russia

${ }^{t}$ Also at Giresun University, Faculty of Engineering, Giresun, Turkey

${ }^{u}$ Also at Graduate School of Science, Osaka University, Osaka, Japan

$\checkmark$ Also at Hellenic Open University, Patras, Greece

w Also at Horia Hulubei National Institute of Physics and Nuclear Engineering, Bucharest, Romania

$x$ Also at Institucio Catalana de Recerca $i$ Estudis Avancats, ICREA, Barcelona, Spain

$y$ Also at Institut für Experimentalphysik, Universität Hamburg, Hamburg, Germany

$z$ Also at Institute for Mathematics, Astrophysics and Particle Physics, Radboud University Nijmegen/Nikhef, Nijmegen, Netherlands

aa Also at Institute for Nuclear Research and Nuclear Energy (INRNE) of the Bulgarian Academy of Sciences, Sofia, Bulgaria

ab Also at Institute for Particle and Nuclear Physics, Wigner Research Centre for Physics, Budapest, Hungary

ac Also at Institute of Particle Physics (IPP), Canada

ad Also at Institute of Physics, Academia Sinica, Taipei, Taiwan

ae Also at Institute of Physics, Azerbaijan Academy of Sciences, Baku, Azerbaijan

af Also at Institute of Theoretical Physics, Ilia State University, Tbilisi, Georgia

ag Also at Instituto de Fisica Teorica, IFT-UAM/CSIC, Madrid, Spain

ah Also at Istanbul University, Department of Physics, Istanbul, Turkey

ai Also at Joint Institute for Nuclear Research, Dubna, Russia

aj Also at LAL, Université Paris-Sud, CNRS/IN2P3, Université Paris-Saclay, Orsay, France

${ }^{a k}$ Also at Louisiana Tech University, Ruston LA, United States of America

al Also at LPNHE, Sorbonne Université, Paris Diderot Sorbonne Paris Cité, CNRS/IN2P3, Paris, France

am Also at Manhattan College, New York NY, United States of America

an Also at Moscow Institute of Physics and Technology State University, Dolgoprudny, Russia

ao Also at National Research Nuclear University MEPhI, Moscow, Russia

ap Also at Physics Department, An-Najah National University, Nablus, Palestine

${ }^{a q}$ Also at Physikalisches Institut, Albert-Ludwigs-Universität Freiburg, Freiburg, Germany

ar Also at School of Physics, Sun Yat-sen University, Guangzhou, China

as Also at The City College of New York, New York NY, United States of America

at Also at The Collaborative Innovation Center of Quantum Matter (CICQM), Beijing, China

au Also at Tomsk State University, Tomsk, and Moscow Institute of Physics and Technology State University, Dolgoprudny, Russia

av Also at TRIUMF, Vancouver BC, Canada

aw Also at Universita di Napoli Parthenope, Napoli, Italy

* Deceased 